\title{
Generation and Propagation of Finite-Amplitude Waves in Flexible Tubes (A)
}

\author{
Jensen, Leif Bjørnø
}

Published in:

Acoustical Society of America. Journal

Link to article, DOI:

$10.1121 / 1.1981915$

Publication date:

1972

Document Version

Publisher's PDF, also known as Version of record

Link back to DTU Orbit

Citation (APA):

Jensen, L. B. (1972). Generation and Propagation of Finite-Amplitude Waves in Flexible Tubes (A). Acoustical Society of America. Journal, 52(1A), 145-145. https://doi.org/10.1121/1.1981915

\section{General rights}

Copyright and moral rights for the publications made accessible in the public portal are retained by the authors and/or other copyright owners and it is a condition of accessing publications that users recognise and abide by the legal requirements associated with these rights.

- Users may download and print one copy of any publication from the public portal for the purpose of private study or research.

- You may not further distribute the material or use it for any profit-making activity or commercial gain

- You may freely distribute the URL identifying the publication in the public portal

If you believe that this document breaches copyright please contact us providing details, and we will remove access to the work immediately and investigate your claim. 


\title{
The Eighty-Third Meeting of the Acoustical Society of America
}

Statler Hilton Hotel • • Buffalo, New York

TuESDAY, 18 APRIL 1972
- 18-21 April 1972

\section{Session A. Speech Communication I : Perception and Confusion}

\author{
Arthur S. Abramson, Chairman
}

Department of Linguistics, University of Connecticut, Storrs, Connecticut 06268

\section{Contributed Papers}

\begin{abstract}
A1. Certain Speech/Nonspeech Perceptual Contrasts. Dorothy A. Huntington and Carl G. Müller, Hearing and Speech Science, Stanford University, Stanford, California 94305. - The effects of changing stimulus durations were examined for speech and nonspeech signals. The aperiodic initial portion of the syllable [Sa] was cut by progressive 7 -msec decrements thus generating stimuli perceived as progressing, as expected, through several categories from unvoiced sibilant to voiced stop. These stimuli were presented in reversed sequential orders to listeners for identification. Two similar sets from a second talker were randomized and presented to 36 judges for identification and for discrimination. Additional stimuli of similar time course prepared from square gated white noise followed by a pulse train were judged for discriminability. Analyses showed that context effects in the sequential identification tasks were small. Discrimination scores reflected response preferences involving order of presentation for both speech and noise stimuli. Comparison of identifications and discriminations shows increasing evidence of categorical perception as category size diminishes or duration decreases. Discrimination and detection curves for speech and noise stimuli show marked constrasts. [We gratefully acknowledge the assistance of Earl D. Schubert.]
\end{abstract}

A2. A Cross-Language Study of Perceptual Asymmetry. T. F. Myers AND J. J. WOLF," Depariment of Psychology, University of Edinburgh, Great Britain.-Recent findings that the speechdominant hemisphere is specialized at the level of distinctive feature analysis entail a prediction that the processing of acoustic cues embodying a feature distinction in a particular language is asymmetric for "native" listeners, but not asymmetric for nonspeakers of the language if their own language does not employ the contrast. Russian- and English-speaking listeners were presented dichotic sequences of syllables for identification of the consonants, i.e., the stops, categorized in Russian, but not in English, as palatalized or nonpalatalized. The results are discussed in the context of a recognition model equipped with a "filter" for feature selection.

*Now at Bolt Beranek and Newman, Inc., Cambridge, Mass. 02138.

A3. Perceptual Features of Nine English Consonants Determined by Choice Reaction Time. Frederick WEINER and Sadanand SingH, Ohio University, Athens, Ohio.-Nine English consonants were presented in pairs for the judgments of same/different to 10 subjects. Subject's choice reaction time (CRT), in making the above judgment, was considered to represent the interpoint distance of the criterion phoneme in subjects' perceptual space. A multidimensional analysis (IND-SCAL) showed that the magnitude of subjects' CRT for determining the sameness or differentness of a phoneme pair was governed by the distinctive features of these sounds. The features retrieved from a three-dimensional analysis were (1) sibilant, (2) voicing, and (3) place. It was further shown that phoneme pairs distinct by zero, one, two, or three feature differences had significantly different CRTs. Pairs having a "zero" feature difference had significantly greater CRTs than pairs different by one-three features. Similarily, pairs with a one-feature difference had significantly greater CRTs than those with a three-feature difference. Within one-feature comparisons, CRT associated with sibilant was shortest following by the features place and voicing. This indicated greater distinctiveness of sibilant than place and voicing. Voicing showed the longest CRT, thus indicating minimal perceptual distinctiveness.

A4. A Search for the Perceptual Features of the 29 Prevocalic Hindi Consonants. Sadanand Singh and Kala Singh, School of Hearing and Speech Sciences, Ohio University, Athens, Ohio 45701.--The 29 consonants of Hindi were recorded prevocalically with the vowel $/ \Lambda /$. Each consonant was included in proportion to its statistical probability in Hindi. Thus, while $/ p /$ was included five times, $/ k / 14$ times. Ten native speakers of Hindi listened the stimuli in each ear separately in five $S / N$ ratio conditions. Listeners responded in an openchoice manner. The responses were written in Devnāgri script. The tallies were made for all the errors which were then averaged for each stimulus consonant across the 10 subjects. The analysis of the 10 matrices (five $S / N$ ratio $X$ Ears) by IND-SCAL method provided best interpretation in fivedimensional space. The perceptual features obtained were best described in articulatory terms. The first two dimensions were interpreted as voicing, aspiration, and sonorant with further interpretation of sonorant as: relrofleclion, nasality, laterality, and semivowel. A subset of 22 consonants, for which place of articulation was phonogically distinctive, was further analyzed. The analysis yielded perceptual features (with articulatory nomenclature) fromi/back and palatal. [This work was partially supported by a grant from NIH.]

A5. Perception of Segments of Spoken (Hindi) VowelConsonant-Vowel Syllables. S. S. Agrawal, Central Electronics Engineering Research Institute, Pilani, India, AND 
S. K. Gupta and Rais Ahmed, Department of Physics, $A$. M. U. Aligarh, India.-This study presents some results of an experiment conducted to determine the effect on perception of deleting different numbers of $10-\mathrm{msec}$ segments from the initial and final parts of VCV syllables. Eight stop consonants /p,t,t,k,b,d,d,g/ and two affricates /ts,dz/ were combined with three pure vowels $/ \mathrm{i}, \mathrm{a}, \mathrm{u} /$ to produce $30 \mathrm{VCV}$ stimulus words. An electronic gating apparatus was developed to present sequential segments of initial and final portions of these syllables. The VC and CV stimuli so obtained were used for two separate listening tests and the responses were analyzed for individual consonants as a function of time. Perceptual phoneme boundaries were found from the response curves and compared with the acoustical phoneme boundaries obtained from sonagrams. The results indicate that the transitions of initial and final vowels terminating in the consonant are of maximal importance for the recognition of intervocalic consonants. The data indicate that the plosive burst plays a more important part in the recognition of unvoiced stops than in the case of voiced stops. The plosive gap was found to play a greater role in the recognition of voiced stops. The affricates were found to utilize all the acoustic events for their recognition. The study of errors showed that for intervocalic consonants, the voicing feature was not recognized in the early part of the initial transition and in the end part of final transition.

A6. Articulatory Rate versus Acoustical Invariants in Speech Perception. A. Quentin Summerfield and Mark P. HaGGARD, Department of Psychology, The Queen's University of Belfast, Belfast BT7 INN, Northern Ireland.-Voice onset time (VOT) has proved in many languages to be a useful descriptive variable with which stop consonants may be classified in respect of the distinction voiced/unvoiced. The question arises as to whether this variable also has fundamental perceptual relevance. Experimental evidence suggests that if VOT as such is registered in preception, then it must be registered in a fashion relative to other ongoing temporal events; for example, we have shown that perception of voicing depends on over-all syllabic rate. However, a separate effect of the rate of transition of the first formant from the onset of voicing to the steady state in the vowel is also evident. Stevens and Klatt (1971) proposed that the presence of a significant and rapid spectral change in the $F 1$ region at the onset of voicing was the positive cue for voicedness. It is possible to go further and attempt to separate the effects of the extent, duration, and rate of the first formant transition. In such a synthesis experiment where VOTs were held constant, we obtained differences in voiced/unvoiced responses that supported Stevens and Klatt's suggestion that VOT is not the real perceptual cue, only appearing so because of the tied variables mentioned. Our results further indicate that there is little or no effect of the extent or duration of the transition, but that a critical rate of transition for perception as optimally voiced may be operating.' The implications of these findings for feature detecting mechanisms in speech perception are discussed. [Work supported by J.S.R.U.U.K.]

A7. Some Perceptual Mechanisms in Speech Processing. Mark P. HaGGard and A. Quentin Summerfield, Department of Psychology, The Queen's University of Belfast, Belfast $B T 7$ 1NN, Northern Ireland.-Enough is known about the acoustical cues in speech to enable highly intelligible synthesis from simple algorithms, but, in most cases, the most valid expressions of cues from the point of view of perception and the mechanism of detection, weighting, and decision are not known. Experiments that manipulate several cues and the phonetic context offer some hope of charting information flow through the human perceptual process and providing detailed block diagram models. Several examples are given. Perception of voicing in initial stops depends upon the place of articulation as well as the traditional voicing cues such as VOT-i.e., the boundary VOT value differs with place. Two separate experiments show that this effect is carried not by acoustical place information but by a perceptual decision about place, logically prior to the voicing decision. But, in another context effect, dependence of consonant place upon the adjacent vowel, the effect appears conditioned by the acoustical vowel information and not by the decision as to linguistic identity. Implications for perceptual theory and speech recognition are pointed out. [Work supported by J.S.R.U.-U.K.]

A8. Stress Patterns and Types of Perceptual Errors. ZinNY Bond, Department of Linguistics, University of Alberta, Alberta, Canada.-The suggestion has of ten been made that stress serves an organizing function in speech perception. The present study attempts to investigate this idea by examining the relationship between stress patterns and the frequency of occurrence of substitution errors and order errors in the perception of obstruent clusters. Eighteen disyllabic CVCCVC nonsense words were selected to serve as stimuli. Only the medial consonant cluster was varied so that all combinations of $\mathrm{p}, \mathrm{t}$, and $\mathrm{k}$ with $\mathrm{s}$ were presented under three different stress patterns: stressed first syllable, stressed second syllable, and equal stress on both syllbales. Preliminary results indicate that, although the number of correct responses is the same under all conditions of stress, the ratio of ordering errors and substitution errors is different for the three stress conditions.

A9. Consonant Confusions in Patients with Sensorineural Hearing Loss. R. C. BILger, M. D. WANG, AND W. H. Jesteadt, Bioacoustics Laboratory, Eye and Ear Hospilal, University of Pittsburgh, Pittsburgh, Pennsylvania 15213.Confusion matrices were obtained for 22 patients on four sets of nonsense syllables, using a forced-choice procedure. Each syllable set considered of 16 consonants in combination with the vowels / $i, a, u /$ either in CV or VC form. Syllables were presented at a comfortable listening level approximately $40 \mathrm{~dB}$ above the audiologic SRT. Over-all performance varied as a function of syllable set, vowel, and hearing loss. The confusions were analyzed by two multidimensional scaling procedures, MDSCAL and IND-SCAL. In addition, an iterative feature analysis of transmitted information was performed, in which the feature system of Miller and Nicely and Chomsky and Halle were compared. [Research supported by grants from SRS and NINDS.]

A10. Effects of Broad-Band Masking Noise on Consonant Confusions. M. D. Wang, R. C. Bilger, and W. H. Jesteadt, Bioacoustics Laboratory, Eye and Ear Hospital, University of Pittsburgh, Pennsylvania 15213.-Perceptual confusions were obtained at six speech-to-noise ratios ranging from -10 to $+15 \mathrm{~dB}$, at over-all noise levels of $50,65,80$, and $95 \mathrm{~dB}$ SPL. Stimuli were four sets of CV and VC nonsense syllables formed by combining all English consonants with the vowels $/ \mathrm{i}, \mathrm{a}, \mathrm{u} /$. Noise levels of 80 and $95 \mathrm{~dB}$ resulted in poorer discrimination, particularly at moderate speech-to-noise ratios. Performance also varied as a function of vowel, syllables with /u/ being consistently better discriminated than others. An iterative feature analysis of transmitted information revealed that in both the Miller and Nicely [J. Acoust. Soc. Amer. 27, 338-352 (1955)] and Chomsky and Halle [Sound Pattern of English (Harper \& Row, New York, 1968)] feature systems, voicing accounted for the greatest relative transmitted information. [Research supported by a grant from SRS]. 
A11. The Verbal Transformation Effect: Consistency of Subjects' Reported Verbal Transformation. NoRMaN J. LASS, Speech and Hearing Sciences Laboratory, School of Medicine, West Virginia University, Morgantown, West Virginia 26506, and Mary E. Tekieli, Department of Communicative Disorders, University of Oklahoma Medical Center, Oklahoma City, Oklahoma 73104.-Continued listening to recorded repetitions of a stimulus has been found to produce perceptual illusory changes in normal listeners. This phenomenon was labeled by Warren [Brit. J. Psychol. 52, 249-258 (1961)] as the verbal transformation effect. The purpose of the present investigation was to study the consistency of subjects' reported verbal transformations in terms of the number of forms and transitions elicited, number of repetitions of the stimulus prior to the subjects' first verbal transformations, types of transformations reported, exact forms employed, and order of reported transformations. Six stimuli, representing variations in meaningfulness and phonetic complexity, were individually presented via headphones to 24 subjects in each of three listening sessions. Results indicate that consistency of subjects' transformations is not equivalent for all measures investigated. Subjects were consistent in the number of forms and transitions and types of transformations that they reported, but inconsistent in the number of repetitions of the stimulus that they required before reporting their first transformations, in the exact forms which they used, and in the order of reported transformations. Consistency as a function of the meaningfulness and phonetic complexity of the stimuli, and the implications of these findings for future research on this phenomenon, are discussed.

TUESDAy, 18 April 1972

Buffalo Room, 9:00 A.M.

\title{
Session B. Nonlinear Acoustics I : General and Air Acoustics
}

\author{
E. G. FubiNI, Chairman \\ Sponsored by Technical Committees on Physical Acoustics and Underwater Sound
}

\section{Contributed Papers (12 minutes)}

\section{9:00}

B1. Guide to the Three Special Sessions on Nonlinear Acoustics. David T. Blackstock, Applied Research Laboratory, University of Texas, Austin, Texas 78712.-As an introduction to the material that is to be covered in the three special sessions on nonlinear acoustics, the principal analytical tools used in the field will be reviewed. These include Earnshaw-Riemann theory, weak-shock theory, Burgers' equation, and Westervelt's method (the Green's function solution of an inhomogeneous wave equation). The review will take the form of an historical account of activity in the field since 1930. Landmark results during this period include the solutions of Fay [(1931). J. Acoust. Soc. Amer. 3, 222-241] and Fubini [(1935). Alta Frequenza 4, 530-581], the $N$-wave solutions of Landau [(1942). J. Phys. Acad. Sci. USSR 6 , 229-230(A); (1945). 9, 496-500], Mendousse's solution [(1953). J. Acoust. Soc. Amer. 25, 51-54] of Burgers' equation, and Westervelt's parametric array [(1960). J. Acoust. Soc. Amer. 32, 934-935(A); (1963). 35, 535-537].

$$
9: 30
$$

B2. Singular Perturbation Methods in Nonlinear Acoustics. D. G. CRIGHTON, Mathematics Department, Imperial College, London, England.-The application of singular perturbation techniques to problems in nonlinear acoustics is demonstrated in two examples. In the first, we show that straightforward perturbation methods, using Lighthill's equation for aerodynamic sound, give a secular series when applied to onedimensional simple wave flow. The nonuniform secular terms are removed by the introduction of expansions in terms of multiple scaled coordinates, and the Earnshaw solution for the shock-free region is recovered. Such an expansion technique is also applied to solve a problem in compound flow. In the second example, the method of matched asymptotic expansions ( $\mathrm{MAE}$ ) is applied to one-dimensional flow governed by Burgers' equation. If the excitation at the origin is time harmonic, Burgers' equation can be solved exactly, and the solution reduced to an intelligible form in the shock-free, saw-tooth, and saturation regions. These simplified approximate solutions are recovered by a direct application of MAE to Burgers' equation, and in work still under completion, it appears that the same process will yield a uniformly valid approximate solution of the analogous problems in two and three dimensions.

$$
9: 45
$$

B3. Nonlinear Theory of Sound Propagation through a Random Medium. M. S. Howe, Mathematics Department, Imperial College, London SW7, Great Britain.-The theory of propagation of weakly nonlinear waves through random media is discussed. The wavefield is partitioned into mean (ensemble average) and fluctuating components, and a nonlinear equation is derived governing the evolution of the mean field. This equation contains a pseudoviscous term that takes account of the irreversible transfer of energy from the mean wave to the fluctuating field. By balancing nonlinear effects against this dissipation, the existence of steady-state shocklike waves is deduced for the mean field, and this is illustrated by reference to the propagation of sound through an atmosphere in which the sound speed is a random function of position and through one subject to turbulent fluctuations. The difficulty encountered in applying the theory to sonic-boom propagation is briefly discussed. [Supported by the Bristol Engine Division of Rolls Royce (1971) Ltd.]

\section{0:00}

B4. Finite-Amplitude Wave Propagation in Relaxing Fluids. R. Klinman and L. Kraus, Drexel University, Philadelphia, Pennsylvania 19104.- The Eulerian equations of motion for wave propagation in a relaxing fluid are transformed to the Lagrangian representation by a Von Mises transformation. A single third-order equation is obtained, which, for certain ranges of the physical parameters (mach number, source frequency, dispersion number, and relaxation time), has uniform, i.e., shock-free, perturbation series solutions. The solution to second order exhibits harmonic generation and spatial beat waves due to velocity-dispersion-induced phase shifts. Examination of the inverse transformation yields additional information on the shock behavior of the system. The region in which the Lagrangian and the previously deter- 
mined Eulerian results [R. Klinman, L. K. Chi, and L. Kraus, J. Acoust. Soc. Amer. 49, 118 (1971)] for relaxing fluids is determined. [Work supported by Office of Naval Research.]

\section{$10: 15$}

B5. Weak-Shock Solution for Initially Distorted Sinusoidal Waves. J. C. Lockwood, Applied Research Laboratories, The University of Texas, at Austin, Austin, Texas 78712.-A theoretical application of Blackstock's weak-shock solution [J. Acoust. Soc. Amer. 39, 1019-1026 (1966)] for the case when a distorted sine wave is assumed as the boundary condition is presented. In Blackstock's analysis, the waveform of an initially sinusoidal wave is expressed as a Fourier series, and the harmonic components are evaluated for ranges up to and including the formation of a decaying sawtooth wave. This paper represents a first step in applying Blackstock's solution for more general boundary conditions. A boundary condition is assumed that consists of a sine wave to which we have added the second-harmonic component of a distortion that is supposed to have taken place prior to the point of application of the boundary condition. The solution is obtained by evaluating the Fourier components of this two-frequency wave as it distorts. [This work was sponsored by the Office of Naval Research.]

\section{$10: 30$}

B6. Numerical Calculation of the Nearfield for Spherically Symmetric Nonlinear Acoustic Flows in an Unbounded Ideal Gas. G. M. Muller, Poulter Laboratory, Stanford Research Institute, Menlo Park, California 94025.-An implicit finitedifference method has been developed for calculating spherically symmetric nonlinear acoustic flows generated by a highintensity periodic source. This method makes it possible to match the conditions prescribed at the source to a lowamplitude solution (in Blackstock's sense) valid at large distances from the source. Numerical computations have been made for pistonlike and sirenlike sources of three different periods and several source amplitudes. Selected graphs of the results will be presented, together with estimates of the errors associated with the method of computation. The numerical results will also be used to show that, in some instances, a low-amplitude solution may be matched directly to a given source even though the conditions for validity of that solution are not satisfied near the source. [Work supported by Aerospace Medical Research Laboratory, Air Force Systems Command.]

\section{$10: 45$}

B7. Acoustics of Thunder. H. S. Ribner AND F. LAM, Institute for Acrospace Studies, University of Toronto, Toronto, Canada.-A phenomenological model of the acoustics of thunder has been briefly explored with the aid of a computer. A conceptually simple program yields the thunder signature in time for a given lightning signature in space. A Fourier transform then yields the frequency spectrum. A few cases of reallife lightning have been tried and plots of thunder signatures and power spectra obtained. The model assumes a lightning stroke to be a spatial distribution of point explosive sources; each one simultaneously generates a disturbance that is idealized as evolving independently of the others. For simplicity, an $N$ wave is used as the elemental wave that evolves by nonlinear distortion from each point. The results suggest that the ensemble average of thunder power spectra, suitably normalized (e.g., total power), resembles the spectrum of the basic $N$-wave building blocks. Additionally, the model, oversimplified as it is, appears to yield pressure-time signatures of thunder that resemble measured signatures.
11:00

B8. Focusing of Finite-Amplitude Cylindrical and Spherical Sound Waves in a Viscous and Heat-Conducting Medium. T. C. Chu and R. Seebass, Department of Aerospace Engineering, Cornell Universily, Ilhaca, New York 14850.- The focusing of cylindrical and spherical pulses of finite amplitude in a medium of small viscosity and heat conductivity has been studied by dividing the region of interest into three parts: converging, interaction, and diverging regions. In the converging region, the flow field is governed by the radial Burgers equation with a small parameter multiplying the term with second derivatives. The method of matched asymptotic expansion is found applicable to this equation with an $N$ wave as an initial condition; a composite solution obtained describes a converging $N$ wave with increasing amplitude and wavelength. The front and rear shocks of the $N$ wave are locally described by Taylor's shock structure. In the interaction region, no small perturbation solution exists for the shocks. However, the flow field between the front and rear shocks satisfies to first order the inviscid linear wave equation, which is solved by the Fourier transform technique. The treatment of the diverging region is similar to that of the converging region, except for trivial changes in the analysis. It is shown that the effect of entropy increase on the failure of the solution at the focus is inconsequential if certain restrictions on the initial strength and wavelength are satisfied.

\section{$11: 15$}

B9. Focusing of an $N$ Wave by a Spherical Mirror. E. P. Cornet and D. T. Blackstock, Applied Research Laboratories, University of Texas at Austin, Ausitn, Texas 78712.An experimental and theoretical study of $N$-wave focusing has been carried out. An $N$-wave source (electric spark) was located on the principal axis of a spherical mirror so as to produce a focus (image point) beyond the center of curvature. The signals before, at, and beyond the focus were received by a wide-band condenser microphone of our own manufacture. Linear theory in the form of the Kirchhoff integral was used to obtain predicted waveforms. Corrections for known nonlinear propagation effects were applied. The data is explained adequately by the composite theory. Diffraction from the mirror's edge plays a prominent role. Before the focus, the reflected signal, a normal $N$ wave, is followed in time by the diffracted signal, which is an inverted $N$. As the focus is approached, the amplitude becomes large and the delay between the two signals tends to zero, giving rise to a $U$-shaped wave, which approximates the derivative of the original $N$. Thus the well-known $\pi / 2$ phase shif $t$ at the focus is explained as the superposition of the two signals. Beyond the focus, the phase shif $t$ of both signals is $\pi$; in addition, the order of arrival is reversed. Nonlinear effects cause distortion of the reflected signal, particularly after the focus, where the initial shape is an inverted $N$. [Work supported by ONR and AFOSR].

\section{$11: 30$}

B10. Propagation of a Weak Oblique Shock through a Diffusing Gas Mixture. Mohsen Sanai, Tau-Yi Toong, Allan D. PIerce, and Richard F. Salant, Massachusetts Institute of Technology, Cambridge, Massachusetts 02139.-The propagation of a weak oblique shock, such as that generated by a supersonic projectile, through a two-component diffusing gas mixture, has been investigated analytically. A linearized ray analysis has disclosed that a two-branched caustic, with associated arête, is formed. The arête and caustic positions have been found as functions of diffusion time, initial Mach number, and molecular weight ratio. 


\section{$11: 45$}

B11. On the Mechanism of Finite-Amplitude Entropic Waves (Part II). Anraham C. Peter, North American Rockwell Corporation, 12214 Lakewood Boulevard, Downey, California 90241 . - This study is a continuation of the work presented at the 81st meeting of the Society in April 1971. The previously derived governing differential equations are integrated under conditions of simple wave motion. It is shown that along the path of the convected entropic perturbations, the pressure and temperature increase whereas the density decreases. Thus, due to the nonlinearity of the solution and the resultant con- stant change of type, the "cold" regions of high density but low temperature and pressure are constantly gaining on the "hot" regions of low density and high temperature and pressure. Under these conditions, low density and high pressure and temperature regions are formed in front of the convected ever steepening "wave." The subsequent collapse of these hot "bubbles" causes a density fluctuation, which varies as a function of the adiabatic exponent $\gamma$ and the local Mach number. For $\gamma=1.4$ and vanishingly small Mach numbers, the density varies as the fourth power of velocity, decreasing to the second power as the Mach number reaches the value of 2.58.

\title{
Session C. Physiological Acoustics I: Contributed Papers
}

\author{
Donald Henderson, Chairman \\ State University of New York, Upstate Medical Center, Syracuse, New York 13210
}

\section{Contributed Papers (12 minutes)}

\section{$9: 15$}

C1. Variation in Eighth Nerve AP Waveform as a Function of Age in Rats. D. E. Crowley, V. L. Schramm, and R. E. SWAIN, Department of Otalaryngology, Washington University School of Medicine, St. Louis, Missouri 63110.-Analysis of computer-summed click-evoked APs recorded from the round window of rats at ages from 1 to 24 months revealed that: (1) The mean visual detection levels of unaveraged APs rises progressively from 6 to 24 months by $16.5 \mathrm{~dB}$. (2) $N_{1}$ amplitude rises from 1 to 12 months, and then declines to 24 months at all but the lowest level ( $50 \mathrm{~dB}$ SPL P.E.) as reported at the previous meeting of this society. (3) Conversion of $N_{1}$ amplitudes to percents of maximum amplitude for each animal eliminates the above age effect, as expected. (4) $\mathrm{N}_{2}$ amplitude increases from 1 to 6 months, but shows only a slight decline to 24 months. (5) The positive peak separating $N_{1}$ and $N_{2}$ remains fairly constant between 1 and 12 months and rises sharply to 18 months, followed by a decline to 24 months. For rats from 1 to 18 months, the general effect of increasing click level is to increase the amplitude of this peak, while at 24 months, the peak decreases and becomes negative at $120 \mathrm{~dB}$ SPL. P.E. (6) $N_{1}$ latency decreases with click level at all age groups, but does not appear to change with age at any but the lowest click level. (7) The temporal separation between $N_{1}$ and $N_{2}$ does show a decrease as a function of advancing age. As a function of click level, $N_{1}-N_{2}$ separation is slightly reduced at moderate levels, compared with its values for low and high levels. [This research was supported by the Deafness Research Foundation].

$$
\text { 9:30 }
$$

C2. Electrophysiologic Study of Descending and Ascending Detection Threshold Discrepancy in Guinea Pig. WilliaM I. Forbes III AND Chester Wilpizeski, Departments of Physiology and Otolaryngology, Thomas Jefferson University, Philadelphia, Pennsylvania 19107.-CM and AP VDLs for continuous trains of clicks were recorded from guinea pigs having permanently fixed round-window membrane electrodes. The experimenter monitored oscilloscope-displayed evoked responses on line by manual adjustment of a motor driven recording attenuator. Bẻkésy-audiometric-type graphical traces were obtained having at least 8-dB discrepancy ("ampli- tude" or envelope width) between descending and ascending VDLs. Comparable VDI s were also recorded photographically by making alternate down-up sweeps across nominal threshold level with no feedback control of the attenuator. Frameby-frame analysis of the film was made off line by measuring stimulus pulse intensity associated with the disappearance (descending VDL) and reappearance (ascending VDL) of the evoked response. No significant overlap of descending and ascending thresholds was found using the photographic method. Statements in the audiologic literature postulating hypothetical auditory nerve processes underlying Békésy trace width are challenged. [Supported in part by NINDS.]

$$
9: 45
$$

C3. The Difference Between the Inner- and Outer-Cell Populations in the Generation of CM and AP. Chang-Yang Wang and Peter Dallos, Auditory Research Laboratory, Northwestern University, Evanston, Illinois 60201.-Literature review reveals that kanamycin creates highly selective damage primarily to the hair cells and the sequence of the damage to the hair cells is relatively regular in such a way that the degeneration of the outer hair cells starts at the basal turn and spreads upward, followed by the degeneration of the inner hair cells starting at the upper turns and spreading basalward, when the dosage per day and the number of days of kanamycin injection increase. The experiment was designed to create a pure cochlear lesion with selective damage only to the outer hair cells at least in the basal turn by careful control of the dosage of kanamycin and to obtain electrophysiological data including CM and AP from a pair of electrodes in the basal turn. The results show that the outer hair cell population is dominant over the inner hair cell population in generating $C M$, and the latter is about 25 to $30 \mathrm{~dB}$ less sensitive than the former in terms of the pseudothreshold of the onset AP. However, a "recruitment"-type phenomenon can be demonstrated for the AP input-output function in that in spite of the sensitivity change the maximum reaches near-normal magnitude. [Supported by grants from the NINDS.]

\section{0:00}

C4. Traveling Wave-Source of Negative Summating Potentials. J. Prazma, Department of Surgery, University of 
North Carolina, Chapel Hill, North Carolina 27514.- Cochlear microphonics (CMs), summating potentials (SPs), endocochlear potentials (EPs), and potassium concentration $\left(\mathrm{K}^{+}\right)$have been recorded from the guinea pig's cochlea. Standard glass microelectrodes and microelectrodes sensitive for $\mathrm{K}^{+}$have been introduced into the scala media. In the first part of the experiments, potentials were measured under different sound pressure levels (SPLs)--80, 90, and $100 \mathrm{~dB}$ at I, II, and III turns. In the second part of the experiments, changes in $\mathrm{K}^{+}$concentration, $\mathrm{SP}, \mathrm{CM}$, and $\mathrm{EP}$ (following anoxia and perfusion of the scala tympani by Ringer's solution, ethacrynic acid, and artificial endolymph) were recorded. Maximum negative summating potentials with their increasing SPL were recorded in loci appropriate for the analysis of those frequencies. Continuous changes in potentials and $\mathrm{K}^{+}$concentration are produced by anoxia and by ethacrynic acid in the scala tympani. In both experiments, when active ion transport is paralyzed, the concentration of $\mathrm{K}^{+}$in in the scala media and the negativity of SP falls. Replacement of perilymph in the scala tympani by a solution with the same concentration of $\mathrm{K}^{+}$as endolymph immediately produces a rapid increase of $\mathrm{K}^{+}$concentration inside the scala media and at the same time an increase in the regative SP. [Supported by NIH grant.]

\section{$10: 15$}

C5. Some Effects of Short-Term Adaptation on Incremental Responses of Auditory Neurons. R. L. SMITH, Laboratory of Sensory Communication, Syracuse University, Syracuse, New York 13210.- Single units in the cochlear nuclei of anesthetized Mongolian gerbils were studied using best frequency tones. The units showed an onset peak and a decay to a steady firing rate in response to $150-\mathrm{msec}$ tone bursts. As in our previous study [Smith, R. L., and Zwislocki, J. J. (1971). J. Acoust. Soc. Amer. 50, 1520-1525], 300-msec tone bursts served as pedestals upon which intensity increments were placed. The time delay from pedestal onset to increment onset was fixed at $150 \mathrm{msec}$. Total onset fring rates have been determined in response to positive and negative increments. The "adapted" response curves so obtained are approximately parallel to the "unadapted" ones measured at the pedestal onset. They are shifted along the stimulus intensity scale relative to the unadapted curves, and the shift increases with the pedestal intensity. This pattern holds for intensity levels of the pedestal up to $15 \mathrm{~dB}$ re the threshold of the units. At higher levels, where the pedestal responses show saturation effects, the slope and the maximum firing rate of the incremental response curves decrease.

\section{$10: 30$}

C6. Second-Order Auditory Pathways to the Superior Olivary Complex in the Rhesus Monkey. N. L. STrominger, Department of Anatomy, Albany Medical College, Albany, New York 12208.-Unilateral stereotactic lesions were made in the cochlear nuclei as follows: restricted to the caudal pole of the dorsal cochlear nucleus (Dc) $(N=2)$; restricted to the posterior end of Dc $(N=2)$; involving Dc and the posteroventral nucleus ( $\mathrm{Pv})(N=2)$; confined to the anteroventral nucleus (Av) $(N=4)$; involving $\mathrm{Av}$ and $\mathrm{Pv}(N=1)$; and involving Av together with $\mathrm{Dc}(N=3)$. Monkeys were sacrificed after 6-17 days. Material was stained by the Nauta-Gygax (1954) technique and as described by Fink and Heimer (1967). Data indicate that fibers from $\mathrm{Dc}, \mathrm{Pv}$, and Av enter the dorsal acoustic stria, intermediate stria, and trapezoid body, respectively. Regarding the superior olivary complex, Dc projects only to the contralateral lateral superior olive (SOL) and fibers are scant; $\mathrm{Pv}$ projects bilaterally to the SOL, preolivary nuclei ( $P O N$ ), and dorsal retro-olivary group, and to the contralateral medial trapezoid nucleus (MTN); and Av projects bilaterally to the PON and medial superior olive (SOM), to the ipsilateral SOL, and to the contralateral MTN. Av efferents terminate on laterally oriented processes of ipsilateral SOM and medial processes of contralateral SOM, and are topographically organized in the vertical dimension. [Supported by NIH grants.

\section{$10: 45$}

C7. Design for a Parametric Study of Neurons in the Inferior Colliculus Using Stimuli of Varying Informational Value. T. W. BarRetT, Department of Physiology and Biophysics, University of Tennessee Medical Units, Memphis, Tennessee 38103.- Using elementary signals defined $\Delta f \cdot \Delta t=\frac{1}{2}$ and $f_{0} \cdot t_{0}=\frac{1}{2} \quad\left(\Delta f=\right.$ bandwidth, $\Delta t=$ duration, $f_{0}=$ center frequency, $t_{0}=$ center period), dichatic stimuli were delivered at cats' ears and varied interaurally by differences in intensity $(\Delta i)$, time of signal arrival $(\Delta \tau)$, and both $\Delta i$ and $\Delta \tau$ in an attempt to at least approximate a parametric study of neurons in the inferior colliculus in the time available for recording. Changes of $\Delta \tau$ and $\Delta i$ with either right or left ear leading in some units had noticeable effects on the profile of poststimulus time (PST) histograms with increased effects at some delays than at others. The registration of $\Delta \tau$ and $\Delta i$ is either by a PST histogram profile or by a change in maximum spike count. Changes in the $f_{0}$ of the $\Delta f \cdot \Delta t$ signal and of $\Delta t$ influenced the PST profile indicating not merely a $\Delta i \cdot \Delta \tau$ trade but a $\Delta f \cdot \Delta t \cdot \Delta i \cdot \Delta \tau=$ a constant function. For some units of the inferior colliculus, therefore, a constant response is elicited by a constant product of four stimulus parameters that may change inversely.

\section{1:00}

C8. Input-Output Functions of Binaural Low-Frequency Medullary Neurons Insensitive to Interaural Time. GEORGE Moushegian and Allen L. Rupert, Research Division, Callier Hearing and Speech Center, and Physiology Department, University of Texas Southern Medical School, Dallas, Texas 75235. - A significant number (about 30\%) of the binaural low-frequency medullary neurons of kangaroo rat are insensitive to interaural time differences. These neurons do not respond in a cyclic manner to the interaural time differences of any frequency within their response areas, but they are activated in a differential manner by small interaural level differences. The discharge rate to a binaural signal for one group of these neurons is greater than the discharge rate to a monaural input. For the other group, the discharge rate to monaural is greater than to binaural stimulation. In the first group, we have binaural facilitation or summation; and in the second, binaural suppression. The most important distinction between these two types, however, is that the facilitative type does not phase lock to monaural or binaural tones, whereas the suppressive type responds in synchrony to either a monaural or binaural sound. [Supported in part by NINDS Grant.]

\section{$11: 15$}

C9. Effects of Medial Geniculate Lesions on Primary Evoked Potentials in Cats. A. Ryan and J. Miller, Deparlment of Physiology and Biophysics, Universily of Washington Medical School, Seattle, Washington 98195.-The click-evoked primary potential was examined in barbiturate-anesthetized cats. Normal and medial geniculate lesioned animals were studied The lesions, electrolytically produced, were placed stereotaxically at various points in the medial geniculate nucleus three days to three weeks prior to electrophysiological recording. The location and extent of medial geniculate destruction was histologically evaluated in each lesioned animal. Bipolar surface potentials were mapped through AI, AII, and Ep. Systematic changes were observed in the cortical evoked 


\title{
83RD MEETING ACOUSTICAL SOCIETY OF AMERICA
}

potential maps. These changes were found to vary with the location and extent of the medial geniculate destruction. The greatest reduction in evoked potentials throughout these cortical areas was observed associated with ventral division lesions. A much smaller effect was observed with medial and dorsal division lesions. In general, the changes may be interpreted to agree with those expected on the basis of anatomical studies of the auditory thalamo-cortical projection system. Changes in the evoked potential map associated with varying ventral division lesions will be described. [This research was supported by NIH grants.]

$11: 30$

C10. Neural Correlates of Habituation of the Cardiac Component of the Orienting Response. Judith C. SANwaLd AND David K. Bliss, State University of New York, Binghamton, New York 13901.-Amplitudes of multiple unit activity (MUA) responses to an auditory stimulus decrease with stimulus repetition. Correlations between neural response decrements and the time course of other indices of habituation [e.g., orienting response (OR) habituation] are not known. We explored the relationship between habituation of the cardiac $O R$ and response changes in the lateral midbrain reticular formation (LMRF), inferior colliculus (IC), and medial geniculate (MG) over successive stimulus presentations. Intracranial electrodes and subcutaneous EKG electrodes were chronically implanted in 16 rats. Subsequently, they were curarized and respirated in a chamber equipped with an overhead speaker. At mean intervals of $90 \mathrm{sec}$, a 5 -sec tone was presented. Twenty successive presentations were made at each of two frequencies. Cardiac rates during the five successive tone seconds were compared with rates for five successive pretone seconds. Most rats showed initial deceleratory responses, which habituated. Others gave acceleratory responses, which were also decremental over trials. The response-generated MUA was analyzed for changes in spike frequency. Amplitudes of MLRF responses either decreased over the 20 trials or showed an initial increment, then a decrement. Smaller decrements occurred in $\mathrm{MG}$ responses and occasionally we saw an initial sensitization. IC responses also tended to decrease over trials. [Supported by a grant from SUNY Research Foundation.]

\section{$11: 45$}

C11. Stimulus Sequencing and the Late Components of the Auditory Evoked Potential. J. B. BeckhaM, JR., Department of Speech and Hearing Sciences, University of Southern Mississippi, Hattiesburg, Mississippi 39401.-Amplitude of the late components of the averaged auditory evoked potential increases as the time between repeated stimuli is increased up to $12 \mathrm{sec}$. The interspersal of tone bursts of different frequencies within the interstimulus time interval was investigated as a method for improving data acquisition rate without sacrificing response amplitude. Series of 500-, 1000-, and $2000-\mathrm{Hz}$ tone bursts were presented singly and in combinations of two and three frequencies at repetition periods of 1 to $8 \mathrm{sec}$. Response records were sorted and averaged accordingto stimulus frequency, yielding one, two, or three averaged evoked potential records. Experimental results indicate that data acquisition rate can be improved by serial presentation of different stimuli without significant loss of response amplitude. [This project was made possible through funds granted by the University of Southern Mississippi Faculty Research Council.]

TuesDay, 18 April 1972

Maple Leaf Room, 9:30 A.M.

\section{Session D. Architectural Acoustics}

\author{
HAROLd R. Mull, Chairman \\ Bell \&o Associates, 80 Danbury Road, Wilton, Connecticut 06897
}

\section{Contributed Papers}

D1. Acoustics in the Design of Synagogues in Israel. F. Michael Strumpf and Zvi Shafrir, Mamash Israel Applied Science Laboratory, $T e l$ Aviv, Israel.-Jewish Temples, of ten called Synagogues, are more than just praying centers. They also form a cultural center. The praying auditorium requires a unique acoustical treatment. This praying auditorium differs from other auditoriums (churches or theaters), in that the audience is an active participant. As such, the auditorium requires a special acoustical design, which considers three important functional regions. The first region is the rear and balcony, which requires a highly absorptive environment. The second is the rest of the audience volume, which requires optimum low reverberant conditions. The third is the front stage, which should reflect acoustically to all other sections. Cultural activities in the adjacent environment have to be considered in the auditorium design as an additional parameter. We will present an example of a design that has used all the above parameters in order to improve the acoustics in a Synagogue.

D2. Acoustical Observations at Blossom Music Center. R. S. Shankland, Department of Physics, Case Western Reserve
Universily, Cleveland, Ohio 44106.-Acoustical observations and measurements have been made at the Blossom Music Center since its inception. The first involved measurements of background noise and wind to determine a suitable site. Over 100 locations near Cleveland were studied before the final location was chosen. The acoustics of the pavillion seating 4500 people has been studied in detail. Reverberation times have been measured at numerous locations showing that it has an excellent reverberation time versus (requency characteristic for orchestral music (2.1 sec in midf requency range). Speech articulation tests have been made, which indicate satisfactory intelligibility even in seats at the rear of the pavillion. The stage shell, designed by Peter van Djik is exceptional in that it provides early reflected sounds throughout the entire pavillion seating area. The pavillion and orchestra shell acoustics make it possible for symphony concerts to be clearly heard by 4500 listeners without sound amplification. For large additional audiences on the lawn sound amplification is provided by a time-delay system, which gives sound that blends perfectly with that coming directly from the stage and the illusion of live sound is achieved for all listeners. For programs other than regular symphony con- 
certs, additional sound amplification is provided within the pavillion.

D3. Influence of Microstructure on Oblique-Incidence Behavior of Fibrous Absorbents. K. AtTenboROUGH, Faculty of Technology, The Open University, Walton, Bletchley, England.A scattering approach, which essentially treats a fibrous absorbent as a suspension of parallel fbers in air, suggests that such absorbents are externally reacting. Furthermore the analysis indicates that the oblique-incidence behavior is greatly influenced by microstructure. Theoretical curves are presented for incidence in planes parallel and normal to the axes of the fibers. These correspond to an anisotropic and an isotropic case, respectively. The influence of random orientation and distributions of fiber radii are also considered.

D4. An Investigation Into the Transmission of Sound Between Rooms with Interconnecting Services Using Statistical Energy Analysis. R. K. Mackenzie and R. H. LyON, Department of Mechanical Engineering, Massachusetts Institute of Technology, Cambridge, Massachusetts 02139. - The theoretical treatment for sound transmission into and out of a duct of infinite and semiinfinite length is given for single and multimodal panel resonance conditions. This theory is then applied to specific cases and compared with the results from experimental measurements in the laboratory in which the effect, upon sound transmission loss, of varying sectional area and shape, duct wall thickness and internal lining is shown. [Work sponsored by National Science Foundation.]

D5. Sound Insulation at Low Frequencies. K. A. MuLHOLLAND AND R. H. LYON, Department of Mechanical Engineering, Massachusetts Institute of Technology, Cambridge, Massachusetts 02139 . - The response of two rooms coupled by a panel has been investigated at frequencies of the order of the lowest room mode frequency. At these frequencies both individual room modes and individual panel modes may be expected to contribute to the transmission of sound through the panel. The role of resonant and nonresonant response both in the rooms and in the panel has been investigated both theoretically and experimentally. It will be shown that transmission is dominated by resonant room modes and nonresonant panel response with some interference from nonresonant room modes.

D6. The Influence of Air Flow Resistance upon the Sound Transmission Loss of Lightweight Concrete Walls. RoBin K. MaCKenZIE, Department of Mechanical Engineering, Massachusetts Institute of Technology, Cambridge, Massachusetts 02139.-A brief account is given of the effect of air flow resistance, porosity, and tortuosity upon the permeability of rigid materials. The relative effects of paint and plaster finishes upon the acoustic impedance of lightweight concrete block walls manufactured from expanded slate and clay aggregates are described, and an indication is given of the most suitable form of wall construction to achieve maximum sound insulation. [Work supported by Cement and Concrete Association.]

D7. A Study of the Effect upon Sound Transmission of Absorption within the Cavity of a Timber Stud/Plasterboard Partition. Robin K. Mackenzie, Department of Mechanical Engineering, Massachusetts Institute of Technolgoy, Cambridge, Massachusetts 02139.-A study has been carried out to measure the effect upon sound transmission loss of placing various forms of absorptive material in the cavity of a timber stud partition. Results shall be presented relating to measurements where the density, rigidity, thickness, area covered, and posi- tion of the absorptive material was varied. These results are compared with theoretical curves and conclusions drawn for the optimum use of absorptive material from the point of view of effectiveness. [Work supported by the Cruden Foundation.]

D8. Impact Noise on Floors. R. D. FORD, University of Salford, Salford, England M5 4WT, AND A. C. C. WARNOCK, Division of Building Research, Ottawa, Ontario, KIA OR6, Canada.A study was made of impulses created by an instrumented standard ISO tapping machine hammer when it strikes various resilient coverings placed on a concrete floor to test the premise that the improvement obtained by using the resilent covering is additive to the basic nature of the floor. In spite of some discrepancies, more marked with carpets than with vinyl, to a close approximation this turned out to be the case. The second part of this study was subjective and was a preliminary attempt to define the parameters of footstep noise upon which subjects base their evaluation. Recordings of footsteps were made in a reverberant room both in its usual condition with a reverberation time of $2-4 \mathrm{sec}$ and with absorbent material added to reduce the reverberation tirne to about $0.5 \mathrm{sec}$, which is more typical of domestic conditions. Subjects were asked to adjust the level of a masking noise until the footsteps were no longer intrusive. A better correlation was found with peak levels of the sound than the long-term rms levels. This suggests that the usual method of normalizing for room absorption may be inappropriate for impact noise problems.

D9. Noise Propagation in Corridors. Gregory C. Tocci and Huw G. Davies, Nepartment of Mechanical Engineering, Massachusetts Institute of Technology, Cambridge, Massachusetts 02139. - As an effort to reduce noise propagation in corridors, a study has been undertaken to evaluate the use of absorbing and shielding baffles in corridors. Both noise attenuation in the corridor itself and also the reduction of noise propagating through open doors into rooms adjacent to the corridor are considered. A discussion is presented of the effectiveness of both the shielding and the absorbing components of the noise reduction for baffles oriented parallel to and perpendicular to the corridor direction. Predictions based on approximate modal summations for the power flow in the corridor are compared with experimental results. [Work supported by NSF.]

D10. Study of the Acoustical Performance of a Landscaped Office. A. C. C. Warnock, T. D. Northwood, and D. N. HenNing, Division of Building Research, National Research Council of Canada, Ottawa, Ontario, KIA OR6, Canada, AND L. W. Hegvold, Bureau of Management Consultants, Department of Supply and Services, Ottawa, Ontario, K1A OT5, Canada.-The occupants of a newly opened landscaped office were subjected to two different masking spectra each day for an extended period. They were polled daily as to their preference and were allowed to comment on the sound masking. Levels of $51 \mathrm{~dB} A$ produced unanimously strong disapproval and rejection. Reactions at $48 \mathrm{~dB} A$, while not so strong, were still negative towards the idea of added noise. Natural sound levels in the office were lowered to about 35-40 dB $A$, but even this relatively quiet condition was preferred to a low level of masking noise ( $45 \mathrm{~dB} A$ ). The effectiveness of the noise for masking speech was measured using the rhyming test described by Griffiths [J. Acoust. Soc. Amer. 42, 236 (1967)] and a test crew. The results obtained showed a predictable relation to articulation index calculations based on $\mathrm{S} / \mathrm{N}$ ratios. Physical measurements were also made to determine the effect of the acoustical ceiling, baffles, and acoustical screens on sound propagation in the landscaped office. The results suggest that there is currently perhaps undue stress on the need for the ultimate in absorption. 
D11. The Problem of Acoustical Specifications for Office Landscape Ceilings. R. N. HAMmE AND D. N. HugGins, Geiger \& Hamme, Inc., Ann Arbor, Michigan 48106.Laboratory mockup measurements of interzone attenuation and subjective judgments of speech privacy can be coordinated through the structure of speech-intelligibility theory to evolve a method of ceiling characterization that offers a sensitive scale for performance evaluation with respect to office landscaping. When the results of such characterizations are interpreted in terms of specular reflection losses incurred during interzone transmission, the sufficient conditions for adequate interzone speech privacy beneath flat ceilings can be stated concisely in terms of material properties and the characteristics of a synthetic background-noise distribution system. Certain of the practical ramifications and logical extensions of this treatment of landscape acoustics are also discussed.

D12. Acoustical Performance of Landscaped Office Ceilings. Hale J. SabINe. Owens-Corning Fiberglas Corporation, Technical Center, Granville, Ohio 43023.-Data is presented on a number of ceiling materials and assemblies tested in the open office mockup at the Geiger and Hamme Acoustical Laboratories. The tests include fat ceilings, baffle configurations, three-dimensional ceilings, and lighting troffers. Performance is rated in terms of speech attenuation over a $5-\mathrm{ft}$ high screen, and by subjective listening tests. Relations between attenuation, specular reflection loss, and reverberation room absorption coefficients for flat ceiling materials are discussed.

TUESDAY, 18 April 1972

Empire AND WaShington RoOMS, 9:30 A.M.

Session E. Engineering Acoustics I : Electret Transducers

\author{
G. M. Sessler, Chairman \\ Bell Telephone Laboratories, Murray Hill, New Jersey 07974 \\ Invited Papers (30 minutes)
}

\begin{abstract}
$9: 30$
E1. Introduction to Electrets. H. J. WINTLE, Department of Physics, Queen's University, Kingston, Ontario, Canada.-A short review will be given of the types of electret in terms of their electrostatic configuration: dipolar, internal space charge (heteropolar), injected space charge (homopolar), and deposited charge (monopolar). The methods used to make thermo-, photo-, electro-, and surfacecharged electrets will be described. The internal fields, short-circuiting effect, stability, limitation due to air breakdown, charge measurement, charge storage, and trapping levels will be discussed.
\end{abstract}

\title{
$10: 00$
}

E2. Electret Transducers: A Review. G. M. Sessler AND J. E. WEst, Bell Laboralories, Murray Hill, New Jersey 07974 . - Condenser transducers made with thick wax electrets were first suggested more than 40 years ago by Nishikawa and Nukiyama [Proc. Imp. Acad. (Tokyo) 4, 290 (1928)]. Electret transducers are now in wide use after the introduction of foil electrets (charged polymer films about 1 mil thick) with long charge lifetimes [G. M. Sessler and J. E. West, J. Acoust. Soc. Amer. 34, 1787 $(1962) ; 40,1433(1966)]$. The charge of the electret replaces the dc bias required in conventional condenser transducers. Charging of the foil is possible with a variety of methods based on thermal, corona-discharge, or electron-beam techniques. Charge retention is particularly good on halocarbons (Teflon FEP, Teflon TFE, Aclar, etc.), where characteristic decay times under normal environmental conditions are measured in terms of decades. Electret transducers have been built as microphones with nondirectional and directional characteristics, as microphone arrays, as earphones, and as electromechanical transducers. Especially electret microphones found widespread practical applications during the past years. Their advantages are excellent frequency response, low distortion, small vibration sensitivity, ruggedness, and low cost.

\section{$10: 30$}

E3. Electrets in Miniature Microphones. Freeman Fraim and Preston Murphy, Thermo Electron Corporation, Waltham, Massachusetts 02154.- The electret capacitive microphone element is ideally suited for miniature applications. The simplicity of its structure leads to a straightforward design even in very small devices. Since the electret foil is the only moving element, and the foil-back electrode spacing the only critical dimension, the electret cartridge is simple in design and extremely rugged. Careful design of the cartridge and associated preamplifier is required to minimize the microphone's self noise. The principal aspects of a miniature electret microphone design are discussed, including the cartridge and the preamplifier, and two examples of small omnidirectional microphones are given. 
E4. Special Electret Transducers. C. W. ReEdyk, Bell-Northern Research, Ottawa, Canada.-Electret transducers are capacitive transducers, which depend on a semipermanent electric charge imbedded in the surface of a dielectric to provide the bias field in an airgap. After many years of experimentation in the laboratory, electret microphones have now become commercially available. The purpose of this paper is to highlight some of the properties of electret transducers and electret materials that make them attractive in some special applications. The basic form, an electret diaphragm stretched over a perforated backplate, is a first-order differential microphone. The response of a first-order differential capacitive microphone, the simple construction, and low sensitivity to shock and mechanical vibration make the electret suitable for use in close-talking microphones, for example, in telephone operator's headset. A simple electret microphone can be used to sense the arrival of the wavefront in a shock-wave tube. The stored charge in the electret has been used in experimental key transducers to produce voltage pulses from 5 to $25 \mathrm{~V}$.

\title{
Contributed Paper
}

$11: 30$

E5. Optimization of a Ridge Backplate for Electret Microphones. H. S. MADSEN, Bell-Northern Research, P. O. Box 3511, Station $C$, Ottawa, Ontario, K1Y 4H7, Canada.-The desired properties for most microphones are high sensitivity, flat frequency response, insensitivity of response to vibration and temperature fluctuations, and long life. The life of an electret is particularly important since the self polarization has a natural decay. It is possible, however, to design an electret microphone that has little variation in sensitivity even though the charge density of the trapped charges diminishes to $\frac{1}{4}$ of its original value. This is possible by controlling every contributing parameter. The parameter that is hardest to control is the tension of the polymer diaphragm and the problem has been resolved by choosing a rectangular backplate with a diaphragm tensioned across parallel ridges. Stray capacitance and electrical load are among the parameters that affect the stability of the microphone, especially when the microphone becomes small as for a lightweight operator's headset. The results of computations and experiments are given.

\section{Session F. Nonlinear Acoustics II : Underwater Applications}

\author{
P. J. Westervelt, Chairman \\ Department of Physics, Brown University, Providence, Rhode Island 02912 \\ Sponsored by the Technical Committees on Physical Acoustics and Underwater Sound
}

Invited Paper (30 minutes)

2:00

F1. Parametric Transmitting Arrays: A Review and Some New Developments. H. O. BERKTAY, Applied Research Laboratories, The University of Texas at Austin, Austin, Texas 78712.*-Since Westervelt [J. Acoust. Soc. Amer. 32, 934-935(A) (1960); also 35, 535-537 (1963)] put forward the concept of parametric acoustic arrays, a great deal of effort has gone into extending his model of a line array with exponential taper to cover primary field distributions that can be realized in practice. Some of these models and their significances will be reviewed and the results of some new work in this field will be presented, with particular reference to the effects in the nearfield of parametric arrays. [Part of the work to be reported is being supported by the Office of Naval Research.]

* On leave of absence from University of Birmingham, Birmingham IS, England.

\section{Contributed Papers (12 minutes)}

\section{$2: 30$}

F2. Nonlinear Attenuation and the Parametric Array. James F. Bartray, Raytheon Company, Portsmouth, Rhode Island 02871 and Peter J. Westenvelt, Applied Research Laboratories, The University of Texas at Austin, Austin, Texas 78712 and Brown University, Providence, Rhode Island 02912.--
The average intensity of a finite-amplitude wave satisfies a simply expressed energy conservation law [P. J. Westervelt, in L. Cremer, Ed., Proc. Int. Cong. Acoust., 3rd (Elsevier. Amsterdam, 1960), p. 316$]$ in terms of the coefficient of smallamplitude attenuation, the first-order pressure, and the virtual source density. This equation when applied to a plane wave yields an equation for the attenuation of the pressure ampli- 
tude, in terms not only of the small amplitude attenuation coefficient, but also the shock formation distance. This latter equation exhibits a smooth transition through the region of shock formation. An approximation applicable to the end-fire array can be developed, and from this a compatible end-fire virtual source distribution. This source distribution results in a directional pattern modified from the low-amplitude Rutherford law [P. J. Westervelt, J. Acoust. Soc. Amer. 35, 535 (1963) ] by a multiplicative factor, which is expressible as a hypergeometric series. This series can be summed exactly on axis, and a simple closed-form expression giving the reduction in sound pressure level due to saturation is found. These results, obtained independently by each of us, are to be published, first in a Letter to the Editor by J. F. B. accepted by J. Acoust. Soc. Amer. and later in greater detail in a joint paper by both of us.

$$
2: 45
$$

F3. High-Intensity Effects in the Parametric Transmitting Array. H. M. Merklinger, Defence Research Establishment Atlantic, Dartmouth, Nova Scotia, Canada.-A method is described wherein the endfire array treatments of Westervelt [P. J. Westervelt, J. Acoust. Soc. Amer. 35, 535-537 (1963)] and Berktay [J. Sound Vibration 2, 439-461 (1965)] may be modified to include the effects of a high-intensity primary wave. An approximate empirical expression for the total acoustic intensity of a finite-amplitude plane wave is employed to determine the source function along the array. It is shown that in the limit of a very intense primary wave, the scattered sound waveform in the forward direction does not depend upon the parameter of nonlinearity The beam pattern (calculated for the case of periodic on/off modulation of the primary wave, only) is shown to narrow as the parameter of nonlinearity is reduced. Various effects related to harmonics of the "difference" frequency are discussed briefly. [This work was carried out at the University of Birmingham under the sponsorship of the Admiralty Underwater Weapons Establishment, Ministry of Defence (Navy).]

\section{3:00}

F4. On the Conversion Efficiency of a Parametric Source. Francis Hugh Fenlon, Westinghouse Electric Corporation, Research and Development Center, Pittsburgh, Pennsylvania 15235.-Plane and spherical wave solutions of the coupled modal form of Burgers' equation, derived by the method of successive approximations, are treated as two asymptotic limits to define the conversion efficiency of a parametric source operating below the shock threshold. These solutions are then related to the dual frequency form of the Bessel-Fubini series, modified approximately to include the influence of viscous absorption over a specifiable range. Examples of parametric source levels computed by means of these relationships are compared with experimental data published in the literature, and the utility of the solutions for predicting the form of the difference-frequency signal as a function of range is also established. Finally, the theoretical expressions that have been derived are summarized in the form of an extremely simple criterion for calculating equivalent parametric source levels, which can readily be extended to sources operating above the shock threshold by means of an approximate finite-amplitude absorption coefficient.

\section{$3: 15$}

F5. Can Explosion Sources Give an Apparent Transmission Gain? ERmine A. Christian, Naval Ordnance Laboratory, While Oak, Silver Spring, Maryland 20910.-The total pressure wave generated by an underwater explosion gradually changes in shape as it propagates out to long ranges. As a result of different attenuation rates in various segments of the high- amplitude explosion pressure wave, the waveform begins to approach a damped sinusoid at some distance from the charge. The effect of varying waveforms on spectral energy distributions has been studied theoretically, with the explosion pressure-time pulse modeled by the function

$$
p(t)=C \exp (-\alpha t) \cos \beta t .
$$

Values of $C, \alpha$, and $\beta$ were derived from empirical functions of explosive charge weight and depth, and range. This analysis shows that the changing waveform may serve to sustain spectrum levels for frequencies in the neighborhood of the explosion's bubble pulse fundamental frequency. Consequently, when the theoretical values for a given charge configuration are combined in the customary sonar equation format, the expected transmission loss can become an apparent transmission gain for selected frequencies. These theoretical results raise the interesting question of whether some of the variability in reported attenuation coefficients might be attributable to the particular explosion sources used in the measurements. [This work sponsored by the Office of Naval Research.]

$$
\text { 3:30 }
$$

F6. Potential Use of Parametric Sonar in Marine Archeology. T. G. Muir and R. S. Adair, Applied Research Laboratories, The University of Texas at Austin, Austin, Texas 78712.Over the past few years, the location and recovery of artifacts buried in marine sediments has been enhanced by the utilization of several technological innovations. Acoustical technology appears to offer a potential application in the high-reselution delineation of archeological sites. Good acoustic penetration of marine sediments, however, usually requires a low-frequency sonar, and this requirement is not compatible with the realization of good angular resolution, at least for systems of reasonable size. Parametric arrays with their low-frequency, high-resolution capability, appear to be well suited to these conflicting requirements. The results of several measurements made with one such array are presented in support of this contention. These results include acoustic propagation and beam pattern data obtained in sea water on a $20-\mathrm{kHz}$ difference frequency radiation generated by the nonlinear interaction of $190-$ and $210-\mathrm{kHz}$ carriers that were transmitted from a 17-in.-diam line-in-cone source. It is also shown that a 5-in. diam aluminum sphere, buried to a depth of 6 in. in a fine sand sediment, was detected with the difference-frequency radiation but not with the carriers. [This work sponsored by the Office of Naval Research.]

\section{3:45}

F7. An Approximate Model for Parametric Acoustic Source Design. Robert H. Mellen, New London Laboratory, Naval Underwater Systems Center, New London, Connecticut 06320, and Mark B. Moffet, Ocean Engineering Department, University of Rhode Ysland, Kingston, Rhode Island 02881.A parametric acoustic source is comprised of a high-amplitude radiated sound field of two frequencies. Because of fluid nonlinearities, this primary field generates a secondary sound field at the difference frequency. Using a simplified model for the primary sound field of a piston transducer, the secondary source levels and directivities generated by such a parametric source have been predicted. The extent of the primary field is assumed to be limited by linear and nonlinear absorption, and this limit is considered to occur in either the collimatedbeam or the spherically spreading portions of the primary field. Numerical values have been computed for a variety of conditions and compare favorably with the experimental data obtained to date.

$$
\text { 4:00 }
$$

F8. Farfield Interactions in the Parametric Radiator. W. L. Konrad, R. H. Mellen, and J. I. Nelson, New London 


\title{
83 RD MEETING A ACOUTICAL SOCIETY OF AMERICA
}

Laboratory, Naval Underwater Systems Center, New London, Connecticut 06320.- In the parametric radiator, the region where most of the generation of the difference frequency occurs can be in the near- or farfield or shared between them depending on the primary frequency, source level, and the projector size. Most of the experiments conducted previously have operated under conditions of predominantly nearfield generation. An experiment is described that is tailored to examine the parametric radiator where the bulk of the generation occurs in the farfield. The experimental source levels and beam patterns are found to be in close agreement with the MellenMoffett model of the radiator. For some conditions, the beamwidth of the difference frequency is in fact narrower than that of the primary pattern and approaches the square of the primary pattern, a result predicted by the model. The application of the model is illustrated by examples of both nearand farfield generation parametric sources.

\section{$4: 15$}

F9. Difference Frequency Parametric Array Using an Exact Description of the Primary Sound Field. J. G. Willette, Applied Research Laboratories, The University of Texas at Austin, Austin, Texas 78712 .- In parametric arrays, the nonlinear interaction between two, harmonic time-dependent waves creates sum and difference frequency radiations. A solution for the pressure field of the secondary radiations has been obtained numerically for interaction in the farfield of a circular piston source [Papers CC4, 80th meeting, and FF7, 81 st meeting of the Acoust. Soc. Amer.]. In the present analysis, this solution is extended to include interaction in the nearfield as well as the farfield of the circular piston. A single intergral expression, which describes the piston nearfield exactly, is derived from the impulse response of a circular piston. The results are compared to experiments involving the amplitude and azimuthal response of a difference frequency radiation. The theoretical results are also compared to other models using (1) plane-wave and (2) spherical-wave approximations of the primary sound field. [This work was sponsored by the U. S. Navy Office of Naval Research.]

$$
4: 30
$$

F10. Underwater Parametric Reception in the Case of a Spherically Spreading Pump and a Plane-Wave Signal. J. A. Shooter, H. O. Berktay, J. G. Willette, J. J. Truchard, AND G. R. BARNARD, Applied Research Laboratories, The University of Texas at Austin, Austin, Texas 78712.-Parametric reception is an economical means of generating a large virtual continuous end-fire array to gain directivity in the detection of low-frequency signals. In this paper, we assume a spherically radiating pump that mixes with a plane-wave low-frequency signal to generate a secondary sound field at the sum and the difference frequencies. A closed-form solution for the radiated secondary sound field is presented and compared with an "exact" numerical solution. Both theoretical methods are shown to be in good agreement with one another and an experiment performed in a fresh water lake. The experiment consisted of a pump mounted on one end of a $50-\mathrm{ft}$ I beam projecting a $90-\mathrm{kHz}$ narrow beam to a receiving hydrophone on the opposite end of the 1 beam. The signal was a $5-\mathrm{kHz}$ fixed source located $150 \mathrm{ft}$ from the center of the I beam. The receiving system was tuned to $(90 \pm 5) \mathrm{kHz}$ and as the I beam was rotated, beam patterns were generated that agree well with the theoretical results. ['This work was sponsored by the Office of Naval Research.]

$$
4: 45
$$

F11. Array of Parametric Receiving Arrays. H. O. Berktay AND T. G. MUIR, Applied Research Laboratories, The University of Texas at Austin, Austin, Texas 78712.--Theory is developed to describe the performance of arrays whose elements consist of parametric receiving arrays. The directivity of the resulting array of parametric receivers is shown to depend upon the separation of elements as well as the separation between pumps and receivers. The theory evolved is validated by comparison to experimental data obtained with a two-element array in a fresh water lake. Electronic steering of this type of array is also discussed. [Work supported by Office of Naval Research.]

\section{$5: 00$}

F12. Experimental Sonar Equipment using a Nonlinear Projector. G. Pearce, Kelvin Hughes, Smiths Industries Lid., Ilford, Essex, England, AND H. O. BERkTAY, University of Birmingham, Birmingham, England.-A sonar equipment consisting of an experimental nonlinear transmitting array and associated electronics together with a conventional commerical sonar receiving and recording system operating at a carrier frequency of $45 \mathrm{kHz}$ was assembled. Acoustic measurements were made on the transmitting array at its primary frequencies of 465 and $510 \mathrm{kHz}$, and at its secondary frequency. Echoes from various targets were recorded when the apparatus was used for horizontal searching. Comparison was made with a conventional system by replacing the parametric transmitter array by an ordinary commercial sonar projector and examining the recorded echoes from the same targets.

TUESDAY, 18 APRIL 1972

GeOrgian Room, 2 :00 P.M.

\section{Session G. Speech Communication II : Physiology of Phonation and Articulation}

\author{
Harry Hollien, Chairman
}

Communication Sciences Laboratory, University of Florida, Gainesville, Florida 32601

\section{Contributed Papers}

G1. Simulated Vocal Cord Motions in Speech and Singing. Ingo R. Titze and William J. Strong, Department of Physics, Brigham Young University, Provo, Utah 84601.-Vocalis muscle and vocal ligament are modeled separately, each by a row of eight masses, with 2 degrees of freedom per mass.
This allows for motions in the direction of the flow and transverse to the flow. Vertical phasing at low pitches and horizontal phasing at high pitches are demonstrated. Parameters, which can be adjusted at liberty, are cord elasticity, ligament tension, vocalis tension during contraction, sub- 
glottal pressure, and the cord boundary. The variable boundary is employed to allow for various statically strained conditions, such as medial compression and open chink. The normal modes of the system are seen by applying either a sudden burst of pressure (such as in a cough) or by displacing one or several of the masses and allowing the motion to come to rest. Chest, middle, and high registers are demonstrated, including a register transition.

G2. Laminagraphic Investigation of Pulse Register (Vocal Fry) Phonation. H. Hollien and E. L. Allen, Communication Sciences Laboratory, University of Florida, Gainesville, Florida 32601.-The physiologic and aerodynamic parameters of modal register phonation have been extensively investigated; not so well researched are these same parameters for the loft (falsetto) and, especially, the pulse (vocal fry) registers. The present study was designed to add to the knowledge being accumulated for the pulse register-in this case, with regard to the relationships of both vocal fold and ventricular fold area and thickness correlates of variation in $f_{0}$. Subjects were seven males and six females, with normal healthy larynges, who could sustain pulse at high and low repetition rates. Coronal cross-sectional laminagrams were obtained by means of the stroboscopic laminagraphic (STROL) technique. It was found that there were no trends of any type for area or thickness change as a function of frequency variation. However, it was found also that the ventricular folds impinge upon, or load, the true vocal folds for this type of phonation. Hence, it is suggested that the ventricular folds may act to damp vocal fold vibration by coupling their passive mass with that of the true folds. Implications relative to theories of phonation will be discussed.

G3. Vertical Larynx Height during Vocal Frequency Change. T. Shipp and R. M. HALler, Speech Research Laboratory, Velerans Administration Hospital, San Francisco, California 94121.- Vertical larynx height was filmed and thyrohyoid muscle activity sampled in two subjects during continuous and discrete-interval vocal frequency change. For continuous frequency change (glissando), the results showed a high positive relationship between larynx height and vocal fundamental frequency $(r=0.93)$ within the modal register, a moderate relationship within the falsetto register $(r=0.42)$, and no relationship within the pulse register $(r=0.01)$. Discreteinterval frequency change showed a similar pattern of larynx height as in continuous change, with the absolute position of the larynx height as in continuous change, with the absolute position of the larynx in the two conditions $\pm 5 \mathrm{~mm}$ at matching frequency points. Thyrohyoid muscle activity level was always lound to be directly related to vertical larnyx position.

G4. Thyrometer-A Method for Observing Laryngeal Control in Speech. Yuki Kakiтa and ShIzuo Hiki, Research Institute of Electrical Communication, Tohoku University, Sendai, Japan.-This paper reports on the "thyrometer," an optoelectric device for observing the laryngeal control in speech through the movement of the thyroid cartilage. Upward and downward movement of the thyroid cartilage tilts the mirror put on the skin surface near the process of the thyroid cartilage of speaker, and swings the light beam reflected by the mirror. The swinging angle is converted into the variation of light intensity through a specially designed optical mask; then the transmitted light beam is focused on the photocell to obtain the voltage monotonically related to the movement. This method allows a highly sensitive recording of the vertical movement of the thyroid (or cricoid) cartilage in speech almost free from the obstruction of utterance with the simpler device. As the time variation of the movement ("thyrogram") corresponds well with the pitch frequency contour in the speech sample in which the change in pitch frequency is considered to be attributed mostly to the laryngeal control, the thyrogram is assumed to show the laryngeal control that underlies the change in pitch frequency. Result of the observation is utilized to estimate the contribution of laryngeal control separately from that of expiratory control in speech, and the nature of the laryngeal control for suprasegmental features is discussed in terms of a physiological model of glottal sound production.

G5. How is Pitch Lowered? John J. OHaLA, Depariment of Linquistics, University of California, Berkeley, California 94720.- Is the pitch of voice lowered (a) by the active contraction of some laryngeal muscles, (b) by the relaxation of those muscles that raise pitch, or (c) by both mechanisms? Evidence will be presented that supports (c). Specifically, electromyographic recordings point to the sternohyoid muscles' participation in pitch lowering, independent of their activity in other speech gestures, e.g., jaw opening. Further, there is evidence of a probable causal correlation between larynx height and pitch of voice (which accounts for the sternohyoids' activity in pitch lowering). Vertical movements in the larnyx, it will be argued, change the pitch of voice by changing primarily the vertical, not the anterior-posterior, tension of the vocal cords. [Research supported in part by the National Science Foundation.]

G6. New Paint-On Electrodes for Surface Electromyography. G. D. Allen and J. F. Lubker, University of Norlh Carolina, Chapel Hill, North Carolina 27514, AND E. HARruson, JR., Research Triangle Instilute, P. O. Box 12194, Research Triangle Park, North Carolina 27709.-A NASA-developed paint-on electrode has been adapted for biomedical applications by the Research Triangle Institute. $10 \mathrm{~g}$ of silver flake are suspended in $10 \mathrm{~g}$ of Duco cement and $1 \mathrm{oz}$ of acetone. This mixture may be stored indefinitely, with occasional addition of acetone for thinning. In electromyographic use, each electrode is formed by placing a small (or large) drop of mixture on the skin, letting the acetone evaporate, laying the bare end of the lead-off wire over the resulting silver spot, placing another drop of mixture over the wire, and letting the resulting "silver sandwich" dry. This process typically takes less than a minute, and the electrode will remain firmly in place for hours. This paper will discuss the properties of electromyographic signals gathered by means of these paint-on electrodes, as compared with those from hooked-wire electrodes inserted in the same muscles, and will mention the potential usefulness of these electrodes for investigating the development of speech and other motor control patterns in children.

G7. Parametric Study of Single Motor Unit Waveforms in Upper Articulatory Musculature. P. F. MacNeilage, H. $M$. Sussman, AND R. J. HaNson, Department of Linguistics, University of Texas at Austin, Austin, Texas 78712.-Waveforms of single motor units were investigated, primarily in four muscles; the genioglossus, geniohyoid, mylohyoid, and anterior belly of the digastric. Units were sampled at various depths up to $25 \mathrm{~mm}$ from concentric bipolar electrodes inserted vertically into the tongue from points inferior to the mandible and near the midline. Units were predominantly of the usual triphasic form although many other types of waveform were observed. Most triphasic potential durations ranged from 5-7 msec, as compared, for example, with 2.5-4 msec from the same muscles with bipolar hooked wire electrodes. Variables determining these waveforms, and differences in waveforms and unit amplitudes from muscle to muscle will be discussed. [This work was supported by grants from the National Science Foundation. $]$

G8. Frequency Control of Single Motor Units in Upper Articulatory Musculature. P. F. MacNeilage and R. K. Szabo, Department of Linguistics, Universily of Texas at Austin, 


\section{RD MEETING - ACOUSTICAL SOCIETY OF AMERICA}

Austin, Texas 78712.-Frequencies of firing of single motor units were investigated primarily in four muscles; genioglossus, geniohyoid, mylohyoid, and anterior belly of the digastric during isometric and isotonic contractions. Isometric contraction was studied as subjects maintained units at regular fring rates by use of feedback provided by auditory and visual displays of the units (motor unit training). Representative results from relatively small samples obtained so far are as follows. Modal firing rates of individual units ranged from about 10 to $\mathbf{4 0}$ impulses per second in different units, with most rates between 20 and 30 ips. The latter values can be compared with values of 10-14 ips from brachial biceps. The frequency range within which regular firing can occur appears to be larger in these units than, for example, in biceps. Differences in typical firing rates from muscle to muscle will be considered. Isotonic contraction in speech gestures was accompanied in some units by firing rates of up to eight times their modal isometric value. [This work was supported by grants from the National Science Foundation.]

G9. Lip and Tongue Pressure Patterns during Syllable Productions. RoBert E. McGlone, Department of Speech Communication, State University of New York at Buffalo, Buffalo, New York 14226, and William R. Proffit, University of Kentucky, Louisville, Kentucky.-Strain-gauge transducers were placed on the lingual side of the dental ridge of the maxilla and mandible and on the labial side of the mandible. These were positioned to record contact pressures along ridges in five locations from the sides and in the midline. Lip and tongue contacts were recorded as subjects repeated VCV syllables containing $/ \mathrm{i} / \mathrm{,} / \mathrm{a} / \mathrm{,} / \mathrm{u} /$, and $/ \mathrm{s} /$. Different pressure patterns were found both between and within syllable utterances. Pressure peaks from the sides of the tongue corresponded to each phoneme while pressure peaks nearer the midline corresponded temporally with the consonant. Lip pressure peaks were related only with the consonant. Tongue tip and midline lip activity showed a definite movement away from the transducer for the entire syllable. The shape of the contact peaks were related to the vowel used in the syllable.

G10. Intraoral Air Pressures Associated with Stop Consonants in Various Phonetic Environments. KenNeth F. Rudek, Bureau of Child Research, University of Kansas, Laurence, Kansas 66044, AND W. S. Brown, JR., Communications Sciences Laboratory, University of Florida, Gainesville, Florida 32601.- - Previous research has demonstrated that intraoral air pressures associated with stop consonants vary as a function of the contextual environment of the consonant (initial, medial, or final syllable position). In the present study, the effect of adjacent phonemes on the intraoral air pressures for the stop consonants $/ \mathrm{p} / \mathrm{h} / \mathrm{b} / \mathrm{,} / \mathrm{t} /$, and $/ \mathrm{d} /$ was investigated. Recordings of peak intraoral air pressures were obtained from five subjects for each of the four stop consonants in the environment of high front vowels, low front vowels, high back vowels, low back vowels, and other consonants. Highest intraoral air pressures were associated with the stop consonants in the consonant environment; the next highest pressures were associated with the high vowel environment while the lowest pressures occurred in the low vowel environment. No differences in intraoral pressures for the four stop consonants were observed in comparison of the front and back vowel environments. These findings can be interpretd as reflecting a coarticulation effect and thereby demonstrate that phonetic environment does affect the magnitude of the intraoral air pressures associated with stop consonants. The data from the present study also support the trend reported in previous studies for higher intraoral pressures to be associated with voiceless consonants.

G11. An Electromyographic Investigation of Articulatory Movements for Bilabials in Normal and Cleft Palate Speech. Minoru Hikano and Noritake Sanui, Department of
Otolaryngology, Kurume University, Kurume, Japan.-Activity of the orbicularis oris, levator veli palatini, and lateral cricoarytenoid muscles for bilabials $(/ \mathrm{p} /, / \mathrm{b} /, / \mathrm{m} /)$ was investigated electromyographically in normal adults and cleft palate speakers. The orbicularis oris muscle presented a marked peak activity prior to the bilabials in the normal subjects. The levator veli palatini muscle were active for the oral sounds and inactive for the nasal as has been expected. However, its activity was not of a consistent level during successive oral sounds. Especially, the levator of ten showed a marked peak activity prior to plosives corresponding to the orbicularis oris. In cleft palate speakers, the peak activity before the plosives was less marked. Instead, the lateral cricoarytenoid muscle presented a marked peak activity, which suggested a gesture for glottal stop. The levator veli palatini muscle of ten presented a similar activity mode as in the normal.

G12. Temporal Characteristics of Velopharyngeal Muscle Function. J. LuBker, University of North Carolina, Chapel Hill, North Carolina 27514, J. LINDQvisT, Institutionen for Taloverforing, Stockholm, Sweden, AND B. FrITZELL, Sahlgrenska Sjukhuset, Goteborg, Sweden.-Temporal characteristics of palatoglossus and palatal levator muscle action in nasal consonant production were studied in seven Swedish speakers. Single motor unit potentials from these muscles were detected by inserted wire electrodes, stored on magnetic tape, and later recorded on light-sensitive paper for analysis. Results suggest that (1) the palatoglossus contracts to lower the palate actively for nasal consonant production; (2) there is a simultaneous relaxation of the palatal levator; (3) the EMG data show considerable temporal variability in the face of quite invariant acoustic data; and (4) in spite of the temporal variability, there is enough consistency to allow the development of a simple model of palatal coarticulation. Specifically, we suggest that for the production of nasals the palatal musculature functions rather grossly. The levator relaxes its activity in a gatelike fashion, allowing a temporal space during which the palate is easily lowered. During the open phase of the gate a slight pull is provided by the paltoglossus to assist in palatal lowering. During this "gating" and "pulling" process the articulators function for the actual production of the nasal. The position of the "gate" and its concomittant "pull" vary temporally with respect to the acoustic signal of the nasal in a highly predictable manner, largely dependent upon intraoral pressure requirements of adjacent phonemes.

G13. Ultrasonic Study of Vocal-Fold Vibration in Normal and Nasalized Vowels. S. L. HamLet, Department of Physiology and Biophysics, Universily of Washington, Seattle, I'ashington 98105.- Nasal coupling reduces the over-all intensity of a vowel, requiring greater vocal effort to match intensity of a nonnasal vowel. The question is to what extent increased vocal effort for nasal vowels is reflected by a change in duty cycle of vocal-fold vibration. Duration of the vibratory open phase was taken as the time within each cycle during which ultrasonic transmission through the larynx was blocked by reflection from the glottal rim. This measurement is consistent over the small range of transducer positions permitting the ultrasonic beam to pass through the approximate vertical and horizontal midpoint of the vocal folds. At matched microphone signal smplitudes, duty cycles differ for normal and nasalized sustained vowels, spoken at the same vocal fundamental frequency. Results are reported for preadolescent boys. [This investigation was supported by a NIH Grant.]

* Current address: Division of Speech and Hearing Science, University of Maryland, College Park, Maryland 20742.

G14. Quantitative Comparison of Perception of Nasality and Cinefluorographic Analysis of the Velopharyngeal Mech- 
anism. Raymond Massengill, JR., Laura R. Love, and Mary Robinson, Speech and Hearing Center, Duke University Medical Center, Durham, North Carolina 27710.-There is a continuing question in clinical literature about what is meant by the term "nasal speech" and about the measurement of this phenomena. Bzoch (1971) and others (Moll, 1968, and Kantner, 1964) insist upon an operational definition, which includes a listener's general perception of acoustic features of the voice quality. Bzoch also states that velopharyngeal insufficiency alone may not be a determinant of hypernasality. This study investigates the relationship of degree of velopharyngeal gap, as determined by cinefluorographic, to listener judgements of hyper- and hyponasality. Two judges rated 30 subjects with a known degree of velopharyngeal gap on a five-point scale. This allowed the judges to assess the voice quality as +3 , severely hypernasal, to -3 , severely hyponasal, with a midpoint of 0 for normal. The velopharyngeal gaps were ranked in three categories for comparison; 0 as normal, 1 to $5 \mathrm{~mm}$ as moderate hypernasality, and beyond 6 $\mathrm{mm}$ as severe hypernasality. The judgments were compared between judges and between judges and degree of velopharyngeal gap. The reliability of the intrajudge ratings were also examined. These results and their importance to research are discussed.

Session H. Shock and Vibration I : Vibration of Solids

Allen J. Curtis, Chairman

Hughes Aircraft Company, Culver City, California 90230

\section{Contributed Papers (12 minutes)}

2:00

H1. Stress-Wave Propagation in a Class of Elastic Rods. M. Cengiz Dökmecr, Department of Theoretical and Applied Mechanics, Cornell University, Ithaca, New York, AND M. ALPD, New York University, New York, New York.-A nonclassical theory of elastic rods of uniform cross sections, descriptive of wave propagation and vibrations, is constructed within the scope of the theory of Cosserat elasticity. A separation of variables solution is sought for the three-dimensional field equations, and a generalized variational theorem is used in the analysis. The effects of transverse shear and normal strains as well as those of rotatory inertia are taken into account. The microrotational motions give rise to new type of waves not present in any of the known rod theories. In particular, the nature of extensional waves is discussed and the uniqueness of solution is established. The results are valid for both nonpolar and Cosserat rods, and contain those of earlier theories as special cases. [Supported by the Office of Naval Research.]

\section{$2: 15$}

H2. Vibration and Buckling of Anisotropic Shallow Shells. J. F. Oyler, The Dravo Corporation, Neville Island, Pittsburgh, Pennsyluania 15225, AND C. L. DYM, Carnegie-Mellon University, Pittsburgh, Pennsylvania.-The free vibration frequencies and the critical buckling loads of certain thinwalled shells of revolution constructed of anisotropic materials-layered or heterogeneous-are studied, the specific geometries being circular cylinders, barrels of positive and negative Gaussian curvature, and spherical sections. The buckling configurations include axial compression and simple radial pressure. In each case the shell boundary conditions are simple supports. The analyses are based upon shallow shell theory, linearized about an initial membrane pressurization state. Using known modal techniques, a frequency determinant is derived. From this determinant studies of the effects of in-plane inertia, of various wave numbers, and of the physical parameters of the shell are carried out. Additionally, results for the static buckling of these shells under axial compression and radial pressure are also obtained, using the kinetic buckling criterion. Parameters studied in the vibration and stability analyses include the thickness-to-radius ratio, initial pressurization, circumferential and axial wave numbers, and the degree of anisotropic asymmetry. The latter effect is studied by the comparison of analyses of two different layered anisotropic materials - each constructed of five equa thickness orthotropic layers with varying orientations. The orientations have been selected to produce varieties of anisotropy-midplane symmetric and midplane asymmetric. The results of these free vibration and stability analyses have produced the following conclusions: (1) the effect of anisotropic asymmetry is so significant that it must be considered; (2) the increased stiffening found for orthotropic cylinders as a consequence of increased thickness ratio and initial pressurization also was found for the general anisotropic family of shells studied.

$$
\text { 2:30 }
$$

H3. Natural Frequencies of Clamped, Generally Orthotropic Rectangular Plates. P. A. LAura AND M. J. MaurizI, Department of Engineering, Universidad Nacional del Sur, Bahia Blanca, Argentina.-This study deals with the determination of frequencies of vibration of clamped rectangular plates using a simple polynomial approximation. The plates are considered to be constructed from an orthotropic material such that the principal elastic axes of the materials are not parallel to the plate edges. A weighted-residual technique is used to solve the differential system, and the change in fundamental frequency is investigated as the material elastic axes are rotated with respect to the natural coordinate system of the plate.

\section{$2: 45$}

H4. Fundamental Frequencies of Noncircular Elastic Hinged Arcs. E. Romanelli and P. A. Laura, Department of Engineering, Universidad Nacional del Sur, Bahía Blanca, Argentina.Free vibrations of elastic, hinged, noncircular arcs have been investigated by several authors. In general, the spatial derivative of the radius of curvature has been neglected. The present study deals with the determination of the fundamental frequencies of several noncircular arcs vibrating in their plane taking into account the variation of the initial radius of curvature with respect to the spatial variables. Several interesting conclusions are obtained.

$$
\text { 3:00 }
$$

H5. Vibration of Statically Deformed Beams and Plates. Huw G. Davies and Saud al Sowayel, Department of Mechanical Engineering, Massachusetts Institute of Techonlogy, Massachusetts 02139.-The response of beams and plates to 
fuctuating loads with high mean values is considered. The static deformation is obtained separately: the vibration of the statically deformed structure is then discussed in terms of the induced static in-plane (membrane) stresses. It is shown that the response of a deformed plate can be predicted by defining an effective uniform tension. Simple expressions are obtained for the loss factor in terms of the impedance of the supports of the beam or plate. Expressions are given for the changes due to the tension of modal resonance frequencies and loss factors (assuming constant boundary impedance). The predicted changes in loss factors agree quite well with some previously published experimental data for very thin plates. The changes for most cases of practical interest, however, are small.

$$
3: 15
$$

H6. The Direct and Reverberant Response of Strings and Membranes to Convecting, Random Pressure Fields. P. LeEhey aNd H. G. Davies, Acoustics and Vibration Laboratory, Massachuselts Instilute of Technology, Cambridge, Massachuselts 02139. - The vibratory response of strings and membranes to convecting random pressure fields is discussed, with particular attention being paid to the traveling wave component of the response. A closed-form Green's function describes the string response. The pressure field is modeled by a summation of random sinusoidal excitations: such a sum, suitably defined, has been shown to represent an ergodic process in a liniting sense. The response of a finite string is shown to divide naturally into a direct response characterized by the convection velocity and decay rate of the pressure field, and a reverberant response characterized by the wave velocity and damping of the string and which exhibits modal resonances. The analysis is extended to include membranes by expanding the lateral membrane response in a modal summation. Each lateral mode is treated analagously to the string. It is shown approximately for small damping that the longitudinal space-time response cross correlation has a reverberant component symmetric about zero time delay and a direct component symmetric about the convection time (spatial separation/convection velocity). These symmetry properties are used to obtain values of the convection velocity from experimental measurements.

$$
3: 30
$$

H7. Damped Lateral Vibration in Axially Creeping Beam with Random Material Parameters. W. N. Huang and F. A.
Cozzarelli, Stale University of New York at Buffalo, Buffalo New York 14214.-It is assumed that the material of the beam is governed by a nonlinear Maxwell model. The temperature and imperfection density and consequently the two inelastic material parameters are taken as random functions of the distance along the neutral axis. A perturbation about the initial axial stress is employed, analogous to the approach used in Cozzarelli, Wu, and Tang ["Lateral Vibration of a Nonlinear Viscoelastic Beam under Initial Axial-Tension," J Sound Vibration 13 (1970)]. As in Cozzarelli and Huang [Effect of Random Material Parameters on Nonlinear Steady Creep Solutions," Int. J. Solids Structures 7 (1971)], the problem is separated into two uncoupled problems-a "random temperature problem" and a "random imperfection problem." Two special cases are fully analyzed:(a) the random parameters are random processes that are only slightly random, and (b) the random parameters are random variables that are largely random. Statistical results are obtained for the lateral velocity, bending moment, logarithmic decrement and circularfrequency.

$$
3: 45
$$

H8. Approximate Equations of Motion for Elastic Rings and Helical Coils of Small Pitch. D. W. Halnes, University of South Carolina, Columbia, South Carolina 29208.-Onedimensional equations of motion are obtained for in-plane deformation of rings. The results also apply to helical coils of small pitch. The equations are obtained from the variational equations of elasticity by expanding the displacements in a Taylor series of the radial coordinate. Truncation of the series leads to a set of two simple equations of motion, capable of describing the first two modes of harmonic wave propagation. These modes correspond to essentially extensional and flexural motion which are highly coupled at long wavelengths. As in straight bars, such a formulation is limited in accuracy to rather low frequencies. The frequency range is greatly extended by including one more term in the truncated series; this leads to three equations of motion and provides for a thickness-shear mode. Comparison is made with the dispersion curves of the recently solved and considerably more complex generalized plane-stress case. From this comparison, the curves from these equations are seen to include the complex segment and to be highly accurate. Boundary conditions are obtained for finite coils and ring elements. ['This work was supported by the National Science Foundation.]

\title{
Session I. Machine Noise I : Noise Measurement in Reverberant Rooms
}

\author{
C. E. Ebring, Chairman
}

Carrier Corporation, Carrier Parkway, Syracuse, New York 13201

\author{
This session has been arranged by the Technical Committees on \\ Noise and Archilectural Acoustics.
}

\begin{abstract}
Discussion of individual papers will be suspended until all papers have been delivered when a discussion of the complete session will be lead by Uno Ingard.
\end{abstract}

\section{Invited Papers}

I1. Noise Measurement in Reverberant Rooms. Richarn WATERHouse, The American University, College of Arts and Sciences, Department of Physics, Washington, D. C. 20016.-The work of Schroeder, Lubman, Maling, Andres, Tichy, Ebbing, and the author is reviewed. Results for the sound fields set up by single tones, multitones, and bands of noise are considered together with the effects of the discrete- and continuous-space sampling of these fields. The variance for such sampling can be calcu- 
lated for the above cases, and gives a basis for sampling in such a way as to get results of prescribed accuracy. Some areas that seem to merit attention at present are mentioned. These include (a) reverberation chamber measurement of the power output of a source radiating a steady single-frequency tone, the accuracy to be expected in such measurements, and the comparability of such results with free-field results; (b) the use of moving reflectors, such as rotating vanes, the improvements they afford, and their effect on the sound field and the power radiation of the source.

I2. The Effect of Stationary and Moving Reflectors and Sampling Techniques on the Accuracy of Sound-Power Determination in Reverberation Rooms. JIRI TICHY, The Pennsylvania State Universily, University Park, Pennsylvania 16802, and Peter K. BAAde, Carrier Corporation, Syracuse, New York 13201.-The paper summarizes the knowledge on different means and techniques for improvement of sound power and pressure averaging in reverberation rooms. Stationary reflectors, properly located in the nearfield of the source and properly oriented with respect to the floor, can contribute considerably to the scattering of the energy radiated by the sound source. The final effect consists of reduction of the dependence of the radiated sound power on the source position. Also, the effect of positioning of the sound-absorbing materials is reduced. Rotating reflector vanes are important for determining the sound power radiated at single frequencies. There are three effects of the rotating vane: First, the radiation impedance of the source depends on the instantaneous position of the vane and, thus, its rotation provides averaging of the radiated power. Second, the motion of the vane causes fluctuations of the sound pressure at each point of the chamber and, thus, reduces the dependence of the sound pressure on the microphone location. Third, the motion of the vane causes a Doppler effect; an originally single-frequency tone becomes a frequency-modulated tone. Thus, a single line spectrum becomes a multiline spectrum, which reduces the spatial variations of the sound pressure and also increases the number of room modes excited. When averaging the sound pressure, different sampling techniques can be used. The paper discusses the practical aspects of some sampling devices and also the problems of the proper choice of instrumentation for electrical signal processing.

I3. Qualification of Reverberation Rooms for Determination of Sound Power. G. C. MALING, JR., IBM Corporation, Poughkeepsie, New York 12603, And C. E. EBBING, Carrier Corporation, Syracuse, New York 13201. - In practice, many noise sources radiate sound having discrete-frequency components. The desire to use a reverberation room for determination of the sound power radiated by such sources has led to the development of a room qualification procedure that may be used to estimate the adequacy of the test setup and the measurement procedure. The theoretical basis for this qualification procedure is discussed. The causes of the various uncertainties in the measurement of sound power are considered, and it is shown that, in effect, the pure-tone room-qualification procedure allows the total uncertainty to be estimated for a monopole-type sound source. The problems involved with discrete-frequency and swept-frequency qualification procedures are discussed, and experimental qualification data for several rooms with and without vanes and other accessories are presented. Methods of generating the multitude of pure tones necessary for measurement of the room qualification are examined, including the advantages and disadvantages of using prerecorded tapes for this purpose. From both theoretical and experimental studies, it can be concluded that a major factor affecting the qualification of a reverberation room is due to the uncertainty in the measurement of a mean-square sound pressure throughout the room. This factor can be minimized by increasing the number of independent samples that one measures during the sound-power estimate by use of either a sufficiently long continuous microphone traverse or by using multiple microphones. At low frequencies (say, below $500 \mathrm{~Hz}$ in a $280-\mathrm{m}^{3}$ reverberation room), the variability of the soundpower output with source position (change in radiation impedance) becomes the major source of the uncertainty of the sound-power estimate if the sampling of the sound field has been accomplished adequately.

I4. Sound-Power Testing Experiences of an Independent Laboratory. Michael J. Kodaras AND Michael W. Blanck, Kodaras Acoustical Laboratories, Elmhurst, New York 11373.-Approximately 150 sound-power level tests have been conducted of unitary and room air conditioning equipment at KAL. This paper submits a complete description of the reverberation rooms used in conducting the tests as well as information regarding the temperature and humidity control systems, room noise levels with and without the control systems operating, and the measurement instrumentation used at KAL. In addition, problems encountered in selecting microphone locations, loading the test specimens, dealing with pure-tone measurements, and other every-day practical problems and their solutions will be discussed. Problems relating to measurement of sound-power level of air handling light troffers, mercury vapor light fixtures, and ballasts will be mentioned. Future changes that will be made to this facility as a result of our experience will be presented.

I5. Statistical Behavior of Source-Receiver Locations in a Reverberant Room. RICHARD H. LyON, Massachusetts Insizitute of Technology, Cambridge, Massachusetts 02139, and Douglas J. InGALLs, Carlyle Compressor Company, Syracuse, New York 13201.-The pure-tone, narrow-band, and $\frac{1}{3}$-oct band probability distribution functions of the SPL for source positions (located on the floor) and receiver positions (located in the interior away from boundaries) were investigated for a random dipole source (Ilg Reference Sound Source), a dipole source (unbaffled loudspeaker), and a monopole source (loudspeaker) for frequencies from 100 to $4000 \mathrm{~Hz}$. The experimental data indicate that an experiment in a reverberant room must be carefully defined. A theoretical model is advanced to explain the observed results. 
I6. The Precision of Reverberant Sound-Power Measurements. David Lubman, D. Lubman \& Associates, Woodlend Hills, Californic 91364.--Determination of sound power in a reverberation room is subject to a random error whose size can be estimated and controlled by the knowledgeable laboratory manager. Under ideal measurement conditions the random error can be expressed as a product of three factors: a frequency-averaging factor governed by spectrum shape and room reverberation time, a spatial-averaging factor determined by the number and separation of fixed microphones or the path shape and size for a traversing microphone, and a rolating-diffuser factor depending on the size and shape of the rotating diffuser in relation to room size and reverberation time. Continuing progress in this field is teaching us to incorporate nonideal measurement conditions into our error estimates. In particular, we report progress in understanding the effects on random error due to (1) finite averaging time, (2) presence of a direct field component, and (3) insufficient modal overlap at low frequency.

I7. Effective Modal Density in a Reverberant Sound Field for Finite Sized Sources. BEn H. Sharp And Kenneth M. Eldred, Wyle Laboratories, El Segundo, California 90245.-Much of the theory concerning the formation of sound fields in reverberant rooms and the measurement of the sound levels produced by these fields has emphasized the statistical aspects. A previous paper [K. M. Eldred, B. H. Sharp, and F. M. Murray, "The Coupling of Finite Sized Sources to a Modal Reverberant Sound Field," presented at the 76th Meeting of the Acoustical Society of America (Nov. 1968), J. Acoust. Soc. Amer. 45, 338 (A) (1969)] discussed the coupling of finite sized sources to a reverberant sound field and showed that there is a strong dependence between sound level and source configuration in certain frequency ranges. This paper will briefly review these findings and extend the work to examine the relationship between the effective modal density and the calculated modal density as a function of source configuration. On the basis of this relationship, the relevance of statistical methods as applied to reverberant sound fields with finite sized sources will be discussed.

I8. Summary of Papers and Open Discsusion. UNO INGARD, Massachusetts Institute of Technology, Cambridge, Massachusetts 02139.-Dr. Ingard will lead the summary and open discussion of the papers presented in this session. Questions and points deserving further discussion will be directed to individual authors, after which questions will be accepted from the floor.

TUESDAY, 18 APRIL 1972

Embassy RoOm, 2:00 P.M.

\title{
Session J. Psychological and Physiological Acoustics : Temporary and Permanent Threshold Shift
}

\author{
R. Carhart, Chairman
}

Northwestern University, Evanston, Illinois 60201

\section{Contributed Papers (12 minutes)}

2:00

J1. Influence of External Ear Acoustics on an Impulse Arriving at the Ear Drum. G. Richard Price, Human Engineering Laboratory, Aberdeen Proving Ground, Maryland 21005.-The effectiveness of acoustic impulses in producing changes in hearing sensitivity varies greatly as a function of pulse characteristics. In the hope of determining why this is so, an ear model of simple geometry [R. Teranishi and E. A. G. Shaw, J. Acoust. Soc. Amer. 44, 257-263 (1968)] was constructed and instrumented with a microphone at the ear-drum position. Impulses from a gunfire simulator, measured in the free field, were compared with the same impulses arriving at the eardrum position. At the ear-drum position, the pulses demonstrated an increased peak pressure, a much longer rise time and a greatly increased duration. A Fourier analysis of the pulses revealed a shift of maximum energy from about 500 $\mathrm{Hz}$ in the free field to about $3.0 \mathrm{kHz}$ at the ear-drum position, which is the reasonant frequency of the external ear. Damagerisk criteria for impulses should probably reffect the frequency content of the impulses and the transformation produced by the acoustics of the external ear.
J2. The Acoustical Impedance of the Guinea-Pig Middle Ear and the Effects of the Middle-Ear Muscles. W. R. J. FunNell and Charles A. Laszlo, BioMedical Engineering Unit, McGill University, Montréal 110, Québec, Canada.-The acoustical input impedance of the guinea-pig middle ear was measured in the frequency range $100-10000 \mathrm{~Hz}$, using a highimpedance volume-velocity source and a probe-tube microphone. The impedance was measured both in the normal ear and with the tympanic membrane removed; the latter measurement permits accurate characterization of the middleear cavities themselves. The data was compared to a slightly modified version of the middle-ear model of Zwislocki [J. Acoust. Soc. Amer. 35, 1034-1040 (1963)], and new parameter values were calculated to match the present data. We also measured the time courses of transient impedance changes caused by spontaneous contractions of the middle-ear muscles of the anesthetized animals. These transient changes were compared to the middle-ear-muscle effects predicted by the model. [Supported by the Medical Research Council of Canada.] 
J3. The Acoustic Behavior of the Outer-Middle-Ear Complex of Man and Guinea Pig. Albert Sinyor and Charles A. LaszLo, BioMedical Engineering Unit, McGill University, Montreal, Canada.-In this study, the acoustic-transmission characteristics of the human and guinea-pig ear canals were compared. According to Wiener [J. Acoust. Soc. Amer. $18,401-408(1946)]$, the pressure response of the human ear canal is similar to that of a tube open at one end and rigidly terminated at the other. This indicates that in man, the middle ear acts as a high-impedance termination and hence it does not load the ear canal. In the guinea pig, however, we have found that the pressure response of the ear canal is affected by the loading of the middle ear [J. Acoust. Soc. Amer. 50, 92 (A) (1971)]. In particular, two maxima were observed in the experimental curves and these were found to correspond to two peaks in the impedance curve of the guinea-pig middle ear. To facilitate the interpretation of the experimental results, a computer simulation study of the outer-middle-ear complex was performed for the human and guinea-pig ears. The responses predicted by the simulated electroacoustic models were very similar to the experimental data. To compare the extent of impedance mismatch between the outer and middle ear in man and guinea pig, the reflection and transmission coefficients at the eardrum were calculated.

\section{$2: 45$}

J4. The Behavioral Effects of Chronic Exposure to Impulsive Noise in Chimpanzees. A. G. KoestLer, D. N. Farrer, G. V. Pegram, and P. R. Krausman, Aerospace Medical Division, Air Force Systems Command, Holloman Air Force Base, New Mexico.-Two young male chimpanzees were exposed to 24 impulsive noises each night for 30 consecutive nights. The subjects' daytime performance on a temporal discriminative task was significantly poorer than pre-exposure performance standards. Although a general trend towards recovery became evident the subjects never fully regained consistent pre-exposure levels of performance. The data suggest that chronic nocturnal exposure to impulsive noise has a significantly detremental effect on the behavioral effectiveness of performing subjects.

\section{3:00}

J5. Temporary Threshold Shift from Low- and HighFrequency Intermittent Noise as a Function of "Off" Time. W. H. Ahaus and W. D. WARD, Hearing Research Laboratory, Universily of Minnesota, Minneapolis, Minnestoa 55455.Eight listeners were exposed via earphones to two different octave bands of noise: $500-1000 \mathrm{~Hz}$ at an intensity of 115 dB SPL, and $1500-3000 \mathrm{~Hz}$ at an SPL of $110 \mathrm{~dB}$. Six separate exposures to each noise were conducted: once to the noise presented continuously, and once to each of five intermittent noise conditions. For the intermittent conditions, "on" time was held constant at 50 msec and "off" times varied between 50 and $450 \mathrm{msec}$. Exposure durations were based on off times (the longer the off time the longer the exposure), such that all exposures contained the same energy. Exposure durations ranged from $3 \mathrm{~min}$ for the continuous exposure to $30 \mathrm{~min}$ for the 450-msec-off condition. TTS 2 was measured after each exposure for three test frequencies within 1 oct above the upper cutoff frequency of the noise. There was a systematic tendency for the mean TTS to increase with off time for the low-frequency exposure, in contrast to a much flatter function following exposure to the high-frequency noise. The results are interpreted in terms of their relation temporally to the latency and relaxation of the acoustic reflex. [Research supported by the Deafness Research Foundation.]
J6. Temporary Threshold Shift from Impulses. H. McRoBerT AND W. D. WARD, Hearing Research Laboratory, University of Minnesota, Minneapolis, Minnesota 55455.Temporary threshold shif ts were measured for seven listeners after exposure to clicks (one every $3.0 \mathrm{sec}$ ) at certain peak levels for varying amounts of time. First, a critical exposure condition was arrived at for each listener, the criterion for this exposure being that combination of peak level and exposure time that would produce $20 \mathrm{~dB}$ of TTS $_{0.5}$ (temporary threshold shift $30 \mathrm{sec}$ after termination of the exposure) at $4 \mathrm{kHz}$. For two of the listeners, exposure at a peak level of $150 \mathrm{~dB}$ for 1 min (20 clicks) was sufficient to produce the required TTS. Four required $152 \mathrm{~dB}$, while the two remaining listeners required $155 \mathrm{~dB}$. Following this, each listener was exposed to clicks at lower peak levels (on different occasions at least $24 \mathrm{~h}$ apart). At each level, the exposure time was increased to maintain equal energy during exposure. For each subject, the lower-level exposure produced significantly less TTS. Thus equal amounts of exposure energy do not necessarily lead to equal auditory hazards, at least for impulse noise.

\section{$3: 30$}

J7. The Temporary and Permanent Threshold Shifts Produced by Three Levels of Impulse Noise. Ronald W. SitLer, Donald Henderson, and Roger P. Hamernik, Department of Otolaryngology, State University of New York-Upstate Medical Center, Syracuse, New York 13210.-Monaural chinchillas (15) were exposed to 50 impulses of either 155 or 161 or $166 \mathrm{~dB}$ peak SPL. The imuplses were generated by a shock tube and had an $A$ duration of $1 \mathrm{msec}$. Quiet threshold was measured at $0.25,0.5,1,2,4$, and $8 \mathrm{kHz}$ using the average evoked response (AER) technique. Recovery was monitored for 31 days. The median PTS for all frequencies was determined by the level of the impulse: the $155 \mathrm{~dB}$ level produced essentially no PTS, the $161 \mathrm{~dB}$ level produced approximately $10 \mathrm{~dB}$ PTS across all frequencies, and the $160 \mathrm{~dB}$ level produced 13 to $35 \mathrm{~dB}$ PST. For moderate to severe TTS, as much as $14 \mathrm{~h}$ were necessary to reach maximum TTS. Unlike PTS results, TTS $_{\max }$ was not strictly determined by the level of the impulse. Specifically, the correlation between the PTS and TTS $_{\max }$ ranged from only $r=0.34$ to $r=0.75$. The results will be discussed in reference to existing damage-risk criteria for impulse noise. [Research supported by the Hendricks Foundation.]

\section{$3: 45$}

J8. Comparison Between Hair Cell Losses and Permanent Threshold Shifts Produced by Three Levels of Impulse Noise. Donald Henderson, Roger P. Hamernik, and W. SitLER, Department of Otolaryngology, State University of New York-Upstate Medical Center, Syracuse, New York 13210. - The cochleas of chinchillas exposed to three levels of impulse noise described in the previous abstract were examined using the surface preparation techniques and Nomarski microscopy. The cochleagrams and AER audiograms 30 days postexposure were compared. The animals exposed to $155 \mathrm{~dB}$ impulses showed no PTS, but all animals showed large and consistent outer hair cell (OHC) lesions at 6 to 12 $\mathrm{mm}$, from the apex. All cochleas had relatively mild and varied inner hair cell (IHC) losses. The 161- and 166-dB exposures produced PTS values ranging from 0 to $45 \mathrm{~dB}$. The cochleagrams exhibited two basic types of lesions: (1) low level losses of IHC and $\mathrm{OHC}$ throughout the entire cochlea; (2) broad lesions of total OHC loss accompanied by less severe IHC losses. The most dramatic results are (1) complete recovery of hearing sensitivity with large cochlear lesions; (2) when there is a PTS, cochlear lesions were found in all animals; 
however, the exact magnitude and location are difficult to specify on the basis of the postexposure audiogram.

\section{4:00}

J9. Cochlear Degeneration Following Impulse Noise Exposure. Roger P. Hamernik, Donald Henderson, and Ronald W. Sitler, Department of Otolaryngology, State University of New York-Upstate Medical Center, Syracuse, New York 13210.-Twenty guinea pigs were exposed to PTS producing impuses of either 166 or $161 \mathrm{~dB}$ peak SPL. The animals were sacrificed at postexposure times of $\frac{h}{2} \mathrm{~h}$ to 50 days, and the cochleas were histologically examined. At $\frac{1}{2} \mathrm{~h}$ postexposure, hair-cell lesions are observed without phalangeal scars. The lesions are bordered on both sides by greatly swollen and distorted hair cells. This damage is observed to increase in extent for up to 30 days. Atrophic changes in the stria vascularis are seen in regions adjacent to hair-cell lesions. Atrophy of the capillaries of the spiral ligament and swelling of the endothelial cells of the vas spiralis appear but are not specific to the hair-cell lesions. Other changes include loss of supporting cells, collapse of the tunnel of Corti, degeneration of the limbus, and loss of nerve fibers. These experiments present a paradox: Over a period of 30 days the cellular damage grows progressively worse while, presumably, hearing sensitivity is recovering.

$$
4: 15
$$

J10. Hearing Impairment in Monkeys after Exposure to Noise. W. C. Stebeins, D. B. Moony, L-G. Johnsson, And J. E. Hawkins, JR., Kresge Hearing Research Institute and Departments of Otorhinolaryngology and Psychology, University of Michigan, Ann Arbor, Michigan 48104.-Following determination of their normal hearing thresholds by operant conditioning methods, old world monkeys were exposed to octave bands of noise centered at 500,2000 , and $4000 \mathrm{~Hz}$ at levels between 100 and $120 \mathrm{~dB}$ SPL for periods of time up to $160 \mathrm{~h}$. Temporary and permanent hearing loss was evaluated daily after each exposure and for a month following the last exposure; the animals were then sacrificed for examination of the inner ear by phase-contrast and scanning electron microscopy. Maximum temporary and permanent hearing loss was found at the center of the exposure band and extended as much as an octave above the center. Temporary hearing impairment of 40-50 dB occurred following an exposure level of $100 \mathrm{~dB}$ SPL for $2 \mathrm{~h}$; no permanent changes were observed. At the other extreme, exposures of $120 \mathrm{~dB}$ SPL, $8 \mathrm{~h}$ daily for a month, produced temporary hearing loss of $90-100 \mathrm{~dB}$ at and above the octave band center, and permanent loss of 60-70 dB as measured 1 month af ter the last exposure. Related histopathology in the cochlea ranged from minimal to fairly extensive hair-cell loss. The size of the lesions was less than anticipated on the basis of the threshold changes. Their location was in general agreement with the place principle. [Research supported by the National Institute of Neurological Diseases and Stroke.]

$$
4: 30
$$

J11. Recovery from Sound Exposure: Comparison of Human Performance and Responses from Single Auditory-Nerve Fibers in the Cat. E. D. Young and M. B. SaChs, Johns Hopkins University School of Medicine, Batlimore, Maryland 21205.-Following moderate exposure to pure tones $(1 \mathrm{~min}$, less than $70 \mathrm{~dB}$ SPL), the discharge rate of cat auditory-nerve fibers in response to near threshold tones is depressed. Recovery to normal (preexposure) firing rate follows a time course well approximated by the simple exponential function $A[1-b \exp (-l / \tau)]$ [Young, E. D., AND Sachs, M. B. (1971). J. Acoust. Soc. Am. 50, 94(A)]. We have measured probability of detection of near-threshold tones in human subjects following similar exposures. The recovery of detection probability is similar to recovery in the neural data. Recovery time constants are usually under $10 \mathrm{sec}$. These time constants decrease as the test-tone level is increased. As exposure level is increased, time constants for neural data increase. Psychophysical time constants increase with exposure level below about $40 \mathrm{~dB}$ SPL; unlike neural time constants they decrease at higher levels. The relationship of these data to TTS will be discussed. [Supported by the National Institute of Neurological Diseases and Stroke and the U. S. Air Force Office of Scientific Research.]

\section{$4: 45$}

J12. TTS Accompanied by Sustained Reduction of AP Amplitudes in Noise-Exposed Guinea Pigs. RiChard P. Maioriello and Chester Wilpizeski, Departments of Physiology and Otolaryngology, Thomas Jefferson University, Philadelphia, Pennsylvania 19107.--Guinea pigs were fitted with permanent recording macroelectrodes having tips located in the auditory-nerve-cochlear-nucleus region. After recording detection levels and input-output functions for clicks or pure-tone bursts under waking conditions using computeraveraging techniques, the animals were exposed to $115 \mathrm{~dB}$ SPL of broad-band noise for $1 \mathrm{~h}$. Postnoise measurements were made within the hour following exposure and for at least four weeks thereafter. VDLs shifted for clicks and midfrequency tonal stimuli, although corresponding suprathreshold AP responses showed sustained reduction of peak-to-peak amplitudes. The altered form of the AP input-output functions is characteristic of nerve fiber depopulation conditions and suggests that damage occurred to the ear in spite of the fact that VDLs returned to prenoise levels.

WEDNESDAY, 19 APRIL 1972

Georgian Room, 9:00 A.M.

\section{Session K. Speech Communication III : Coarticulation, Phonetics, Duration, and Quality}

W. S. Brown, JR., Chairman

Communication Sciences Laboratory, University of Florida, Gainesville, Florida 32601

\section{Contributed Papers}

K1. Coarticulation of Nasality: Evidence from Hindi. R. Prakash Dixit and Peter F. MacNeilage, University of Texas, Austin, Texas 78712.-The temporal scope of nasal coarticulation and its dependence on segment type, syllable boundary, and word boundary was investigated in Hindi, which, unlike English, has contrastive nasality in vowels as 
well as consonants. Various words with nasalized and nonnasalized vowels and nasal and nonnasal consonants and with syllable boundary falling at different places were selected and inserted in the meaningful sentences, which provided preceeding and following word boundaries to the inserted words. Electromyograms were recorded together with simultaneous recording from oral and nasal microphones. It was shown that (1) the effect of coarticulatory nasality stretches beyond two segments on both sides of a nasal-vowel or consonants; (2) the temporal scope of coarticulatory effects was unrestricted by syllable or word boundary; and (3) left-to-right effects were as extensive as right-to-left effects. These results are discussed in relation to results of previous studies. [This work was supported by grants from the National Science Foundation.]

K2. Cinefluorographic Study of Coarticulation of Pre- and Postvocalic Allophones of $/ 1 /$ in Consonant Clusters. S. B. Giles, Speech and Hearing Center, North Dakola State Universily, Fargo, North Dakota 58102.-Lateral cinefluorographic films were taken of three subjects at a speed of 150 frames/ sec. The subjects, natives of the midwestern U. S. and judged to possess a dialect typical of that region, produced words in which an / $/$ allophone was a part of a pre- or postvocalic consonant cluster. Articulatory movements were analyzed by a frame-by-frame tracing of the structural contours through the phonetic sequences under investigation. In prevocalic clusters, articulation of the [1] was shown to coarticulate with the articulatory movement associated with the phonetic element immediately preceding the [1]. The articulation of the phonetic element immediately following the postvocalic [I] coarticulated with the movements associated with the [1]. The degree of overlap or the timing of the sequence of articulatory movements in the clusters was variable.

K3. Coarticulation as an Adjunct to Perception. W. H. Manning, D. S. Beaslex, and T. S. BEachy, Department of Audiology and Speech Sciences, Michigan Slate Universily, East Lansing, Michigan 48823.-- The hypothesis that a unit of speech production would be more accurately perceived as the degree of coarticulation of that unit increased was investigated. Various models of speech production were employed in the creation of an operational definition of "degree of coarticulation." Sixty subjects listened to 50 randomized monosyllabic two-word phrases, presented under six $\mathrm{S} / \mathrm{N}$ conditions $(-10$ to $+15 \mathrm{~dB}$, in 5 -dB steps). The second word of 25 of the items began with an /st/ blend. In the other 25 items, the first word terminated with the morpheme $/ \mathrm{s} /$, while the second word began with the phoneme $/ t /$. The listeners were required to record manually each of the 50 twoword phrases. Mean percentage correct scores for 10 listeners at each $\mathrm{S} / \mathrm{N}$ condition were calculated. The results revealed that perception was more accurate under the condition representing a greater degree of coarticulation (i.e., /st/ blend) These results support the contention that a relationship exists between perceptual accuracy and degree of coarticulation.

K4. Tape Cutting Experiments with Stop Consonants. ELI Fischer-JoRGENSEN, Instilute of Phonetics, Universily of Copenhagen, Denmark.-In Danish words with /p,t,k,b,d,g/ $+/ \mathrm{i}, \mathrm{a}, \mathrm{u} /$, stop bursts, aspirations, and formant transitions have been removed and exchanged. [Danish $/ p, t, k /$ are strongly aspirated (/t/ affricated), $/ \mathrm{b}, \mathrm{d}, \mathrm{g} /$ are voiceless. $] 500$ stimuli were presented to 21 listeners, who had to identify the words. Main results were: For the distinction between $/ p, t, k$ / and $/ b, d, g /$, the small difference in stop burst is insignificant, whereas the aspiration is decisive (a simple pause between burst and vowel is not sufficient). The duration of the aspiration is important, but the start of the vowel (noisy start of higher formants) may be sufficient for the identification of $/ p, t, k /$, particularly before /a/, even with VOT values of 20-30 msec. For the identification of place of articulation the aspiration (affrication) of $/ p, t, k /$ is sufficient except for $/ \mathbf{k} /+/ \mathrm{a} /$, but the burst is also sufficient except for $\mathbf{t}$. In $/ b, d, g /$ the transition is sufficient if it is extensive (e.g., $/ \mathrm{ba} / \mathrm{l} / \mathrm{da} / \mathrm{,} / \mathrm{ga} / \mathrm{hi} / \mathrm{d} / \mathrm{du} / \mathrm{)}$; it is not enough that it points to the locus. The burst is necessary not only in velars before rounded vowels, as previously assumed, but also, for example, in $/ \mathrm{bu}, / \mathrm{di} /$, and /gi/.

K5. Phonetic Coding of Kanji. D. Erickson, I. G. MatTINGLy, AND M. TURvey, Haskins Laboratories, New Haven, Connecticut 06510 and Department of Linguistics, University of Connecticul, Storrs, Connecticut 06268.-An experiment in the recall of visually presented Japanese Kanji ideograms suggest that Kanji may, like alphabetic words, be encoded phonetically, despite their lack of phonetic structure. The experiment, based on Kintsch's and Buschke's (1969) paradigm, assumed that similarity of items in a list increased errors in recall. Four lists were prepared, each containing 16 different Kanji. The first included phonetically similar pairs of characters; the second, semantically similar pairs; the third, visually similar pairs; the fourth was a control list containing no similar pairs. The subjects, 10 native speakers of Japanese, were presented with randomly ordered versions of each list, at one character per second. After a subject had seen an entire list, he was presented with a cue character selected from the list, and asked to recall the character that had been presented immediately before the cue. Confusion in recall was significantly greater for the phonetic list than for the other lists. These results strengthen the hypothesis that regardless of structure, visually presented linguistic items are, like speech itself, phonetically processed.

K6. A Spectrographic Study of Allophones of Voiceless Stop Consonants in American English. R. MONSEN, M. MOLTER, AND N. Umeda, Bell Telephone Laboratories, Murray Hill, New Jersey 07974.- The times of closure and aspiration in $/ \mathrm{p}, \mathrm{t}, \mathrm{k} /$ were investigated using spectrograms of naturally read texts. Most obvious effects in the data are the differences between word-initial and word-final stops and between stressed-syllable-initial and unstressed stops. There are several effects of phonetic environment-notably a shortening of closure time when the stop is preceded by a nasal. Wordinitial stops form a class with closure and aspiration times varying proportionately from short duration in function words to long durations for emphatic and prepausal content words with primary stress on initial syllables. Final stops form a class tending toward either long closure times or long aspirations, but not both, depending on the immediate phonetic context. Inside the word, the stops form more tightly grouped classes, dependent on the lexical stress and phonetic environment. In general, the durations of closure and aspiration of the voiceless stops are predictable from the identity of the consonant and from its phonological context.

K7. On Significance of Allophonic Variation in Voiceless Stops; Some Results of Vocal-Cord Instrumentation and Speech Synthesis. C. H. Coker and N. Umeda, Bell Telephone Laboratories, Murray Hill, New Jersey 07974.-A study of allophonic variations of voiceless stops in American English was made by transillumination of the glottis and by modeling in speech synthesis. Variants of phonemes are largely ignored in linguistic studies, on grounds that they are nondistinctive- 
i.e., insignificant in distinguishing the words that a phoneme string represents. In speech synthesis, however, we find the selection of allophones more crucial. Durations of closure and aspiration time of consonants in otherwise identical phoneme sequences can indeed force clear choices between such phrases as "the spit/this pit," "at all/a tall," etc. In measurement of vocal cord area by transillumination, data on the consonants $/ p, t, k /$ show the glottis remaining open wide during the plosive burst and most of the aspiration of strongly aspirated stops, then closing rapidly to begin voicing. In weakly aspirated stops, the cords are kept closed, or nearly so, as if there were simultaneously articulated glottal stops. In stressed-syllable-initial /st/, the cords open wide during /s/ but close during $/ \mathrm{t} /$, reaching closure in time to suppress the burst and aspiration. Since the cords are apparently capable of opening or shutting in 30 or $40 \mathrm{msec}$, variants from 0 to $100 \mathrm{msec}$ in aspiration time must be expressly controlled rather than accidental. Thus we see aspiration-time allophones as systematic (Monsen, Molter, and Umeda, previous abstract), explicitly selected and important for distinguishing word sequences. Whether or not they are nondistinctive, these allophones are not linguistically insignificant.

K8. Some Determinants of Vowel Duration in Swedish Words. BJörn E. F. Lindalom And Karin Rapp, Department of Phonetics, University of Stockholm, and Department of Speech Communication, Royal Institute of Techology $(K T H)$, Stockholm, Sweden.--Observations on the systematic variation of vowel duration in Swedish words as a function of word length, position, and stress are presented. The results reported are similar to the data presented in a previous study [Lindblom, STL-QPSR 2, No. 3, 1-5 (1968)] in that the compensatory adjustment of vowel duration is found to be only partial with respect to word length. Reinvestigating the problem quantitatively we have developed a formula around the assumption that "effective" word length is equal to "actual" word length raised to a power. Since the exponent is always $<1$ the actual number of syllables per word seems to be "underestimated." We shall present several types of evidence that appear to support this suggestion. First of all, an accurate description of vowel durations in word-initial positions will be obtained. Second, in combination with a specific interpretation of the role of position, the introduction of the power transformation paves the way for a natural account also of the noninitial data, e.g., the so-called "finallengthening" effect. [This research was supported by the National Institutes of Health under a research grant.]

K9. Vowel Duration in Polysyllabic Words in American English. N. UMEDA, Bell Telephone Laboratories, Murray Hill, New Jersey 07974. - The durations of vowels in polysyllabic words were studied from natural readings of texts by several speakers of various dialects. Durations of vowels in prepausal and word-final stressed syllables follow the same rules as those of monosyllabic words. They are affected strongly by pitch manipulations that reflect the word's syntactic position and semantic value [cf., N. Umeda and C. H. Coker, J. Acoust. Soc. Amer. 49. 123(A) (1971)]. Vowels in other positions in polysyllabic words are comparatively free of higher-level lengthening phenomena; durations are well accounted for by purely phonological conditions. Data are grouped into the following classes: (1) individual vowels with primary stress in non-word-final syllables; (2) vowels in word-initial unstressed syllables; (3) vowels in post-stressed, non-word-final syllables, and (4) vowels in word-final, unstressed syllables (with subclasses according to the type of ending:-ing, -ness, -ment, -er, etc.). Distribution patterns of data for different speakers are very similar; slight differences in average value are plausibly explained by differences in speech rate. After the above factors are taken into account, the remaining effects in these data from readings of texts do not positively support a direct influence of the number of syllables in a word on the duration of each vowel.

K10. Sensitive Test of Speech Communication Quality Lloyd H. Nakatani, Bell Telephone Laboralories, Incorporated, Murray Hill, New Jersey 07974.--Small differences in the quality of various speech samples, all highly intelligible according to articulation tests, were reliably discriminated by a test which determined how easily the speech samples could be heard against a background of interfering speech. The communication quality of a degraded speech was measured by the difference, $Q=T-T_{0}$, where $T$ and $T_{0}$ were the intelligibility thresholds for the degraded and high-quality reference speech, respectively. An intelligibility threshold was defined as the value of the signal-to-interference intensity ratio, where the signal speech was $50 \%$ intelligible in the presence of interfering speech. $Q$ measures and subjective quality ratings were obtained for speech degraded by low- and high-pass filtering, additive noise, and transmission over a telephone line. The $Q$ measures successfully discriminated differences in speech quality, and they were also monotonically related to the ratings, thereby validating the test for speech quality evaluation. Articulation scores and ratings were not monotonically related, a finding which calls into question the validity of articulation tests for the evaluation of highquality speech samples.

K11. A Normative Study of Listening-Rate Preferences of Adults. Norman J. Lass and Cheryl J. Cain, Speech and Hearing Sciences Laboratory, School of Medicine, West Virginia University, Morgantown, West Virginia 26506.-A paired comparison procedure was employed to determine the listening-rate preferences of 100 adult subjects. The recording of a standard prose passage was time altered by means of a speech compressor to yield nine reading rates: $100,125,150,175$, $200,225,250,275$, and $300 \mathrm{wpm}$. A total of four master tapes were constructed, one for each of the four different orders employed for presentation of the readings. Each tape consisted of 36 pairs $[n(n-1) / 2]$, with each of the nine reading rates appearing eight times on the tape. Each of the four master tapes was played to a different group of 25 subjects for their listening-rate preference evaluations. Results of their evaluations indicate that (1) all but one of the nine rank orders of preferred listening rates were identical for all four subject groups; and (2) the most preferred listening rate for all groups was $175 \mathrm{wpm}$, and the least preferred rate was 100 wpm. Implications of these findings for future research are discussed.

K12. The Instant of a Syllable : Effects of Differing Sentence Frames. Paul C. Boomsliter and George S. Hastings, Jr., State University of New York at Albany, New York 12222, and Warren Creel, Albany Medical College, Albany, New York 12208. - At the 80th convention of this society, we presented a paper indicating that when speaking a simple sentence in time to a metronome, a speaker places one or another specific articulatory action on the timing point within a stressed syllable. Results of an expanded study using 20 readings of each of 38 syllables embedded in each of four different sentence frames ( 3040 samples) indicate that the principle is valid well past the limits of the original study and that different sentence structures produce some differences in the principle's application. 
Session L. Shock and Vibration II : Macrosonics

A. Semmelink, Chairman

IIT Research Institute, Chicago, Illinois 70616

Invited Papers

9:00

L1. Macrosonics and the Ultrasonic Motor. L. BaLAmuth, Research Director, Ulstrasonic Systems, Inc., Farmingdale, New York 11735.- Macrosonics is presented as a part of a complete periodic motion and mechanical vibration spectrum, followed by a resume of the uses of ultrasonic motors, including the category of macrosonic motors that covers a frequency range dipping down into the sonic and subsonic levels. Several features of ultrasonic motor design, to which the author has contributed, are presented and are connected with practical problems that arise in applying such motors in the macrosonic sense. These features include the production of high output motor velocities without benefit of effects normally associated with ultrasonic horns or mechanical impedance transformers; the incorporation into a macrosonic motor of a "flywheel" effect; and the provision of macrosonic energy storage by means of motor elements whose cross section is not limited by the usual quarter wavelength requirements. The elementary properties of macrosonic motors are reviewed together with the relevance of such properties to macrosonic technological applications. Specifically, some of these properties are illustrated in greater detail in reference to joining metals and plastics, as well as to metal deformation. The special areas selected are confined to methods and means arising directly out of the author's work.

\section{$9: 25$}

L2. New Developments in Metal Working Processes. Andrew ShoH, Branson Sonic Power Company, Danbury, Connecticut 06810 .- Recent experiments in sonically assisted metal machining and forming are described. A wide range of vibratory inputs is explored to elucidate the cause and effect relationship. The emphasis is placed on presenting the data in terms of basic physical quantities and on defining the controllable sonic parameters contributory to the process. Specifically, ultrasonically assisted drilling using conventional twist drills is discussed where axial vibratory motion is superimposed on rotational motion of the drill. Novel testing techniques are used to generate and analyze the data. The second part of presentation deals with ultrasonically assisted metal forming where deformation of simple cylindrical specimens is observed as a function of combined static and dynamic stresses. Both studies include a variety of metals.

L3. Ultrasonic Welding for Electrical Conductivity Applications. J. BYroN Jones, Aeroprojects Incorporaled, West Chester, Pennsylvania 19380.-Ultrasonic welding, which produces bimetallic as well as monometallic junctions of integrity at temperatures below about one-half the absolute melting value, is effectively used in electrical conductivity applications such as making wire terminal connections, attaching armature coils to commutator bars, and consolidating stranded copper and/or aluminum wire into solid sections. New aluminum conductivity alloys exhibiting improved physical properties, coupled with the economic lever existent in substituting aluminum for copper, have triggered extensive efforts to utilize the ultrasonic welding process in electrical equipment particularly for the automotive, appliance, and aircraft industries. Harnesses and starting motors are discussed, and ultrasonic welding data for such applications, including equipment size, power, operating parameters, and product quality, are presented.

\section{$10: 15$}

L4. Ultrasonic Cutting of Rock Materials. B. DurLEY, K. E. FeITH, and R. R. Whymark, Interand Corporation, Rockville, Maryland 20750.- Low-Irequency reciprocatory-rotary motion is imparted to a conventional axially vibrated ultrasonic cutter $(18 \mathrm{kHz})$ that is impregnated with diamond grit of average size 0.003 in. The rotary motion is in a plane normal to the cutter axis. Cutting rates in rock materials of $10 \mathrm{ft} / \mathrm{h}$ are exceeded for 1 -in. diam circular holes drilled to depths greater than $1 \mathrm{ft}$. Peak axial amplitude was 1.6 mil and the rotary motion was less than three to-and-fro oscillations per minute. Specific energy of the cutting process was measured to be less than $100 \mathrm{~J} / \mathrm{cm}^{3}$ or less than that involved in the rotary cutters used for oil well drilling. Pulsed excitation at a $50 \%$ duty cycle leads to an $8 \%-10 \%$ increase in drilling rate for the same power input. Self pumping of the abraded material is obtained through subharmonic vibration of the cutter on pulse excitation. Static pressure, peak amplitudes, and rotary motion are optimized. 
L5. A Discussion of Ultrasonic Intensity Measurements as Related to the Radiation Control for Health and Safety Act. S. W. Smith and H. F. Stewa RT, Bureau of Radiological Health, Rockville, Maryland 20852. - The Bureau of Radiological Health is currently implementing an ultrasonics program to develop and evaluate measurement techniques, perform product evaluation, and assess potential hazards with the ultimate goal of determining the need for equipment performance standards under the Radiation Control for Health and Safety Act, Public Law 90-602. The program will include studies of ultrasonic radiation emissions and safety features for industrial, medical, and consumer devices. The initial research effort includes the development of a primary measurement calibration system applicable to plane-wave ultrasonic radiation in pulsed or $\mathrm{CW}^{\prime}$ mode; and the construction of secondary portable measurement devices suitable for field measurements.

\section{$10: 55$}

L6. Ultrasonic Synergism in Biochemistry, Ultrasonic Irradiation of Nucleic Acids and Microorganisms, Applications to Sterilization. R. M. G. Boucher, Wave Energy Systems, Inc., New York, New York 10022, AND A. J. LAST, Ontario Research Foundation, Ontario, Canada.-In this lecture, a review is made of recent work and concepts dealing with the insonation of nucleic acids (DNA, RNA transfer, etc.). Possible implications in the field of genetics are reviewed briefly. The role of free radicals during the insonation of microorganisms is analyzed with particular emphasis on the behavior of highly resistant spore strains. A new sterilization theory is exposed which is based upon synergistic effects observed during the insonation of aqueous glutaraldehyde solutions at reasonably low temperature $\left(54^{\circ} \mathrm{C}\right.$ ). Results obtained with resistant $B$. subtilis spores (including AOAC) are given. Conclusions are drawn regarding the use of this technique in hospitals and industry (continuous sterilization of disposables). Economic aspects of the new synergistic sterilization method are analyzed.

\section{$11: 20$}

L7. Fusion of Plastic Powders with Macrosonics. H. V. Fa1r Banks, Department of Chemical Engineering, West Virginia University, Morgantown, West Virginia 26506.-Both thermosetting and thermoplastic powders were molded into $\frac{1}{2}$-in. diam cylinders by the application of macrosonics plus a nominal constant pressure without the use of external heat. A series of factorically designed experiments were run using ultrasonic intensity, pressure, and time as the variables. The measured length of the fused cylinder of plastic formed in each experiment was used to evaluate the response. All the variables were found to be relatively important to the fusion process. However, only the interaction of time and pressure was found to be important. This interaction had a negative slope coefficient.

\section{Contributed Papers (12 minutes)}

\section{1:35}

L8. Ultrasonic Metal Removal. Janet Devine and W. B. TARPLey, Aeroprojects Incorporated, West Chester, Pennsylvania 19380.-The results effected via ultrasonic excitation of the cutting tool in material removal processes such as lathe turning, boring, and twist drilling are discussed. Single-point cutting with ultrasonic-cutting-tool drive results in increased metal removal rates, improved surface finish, and improved machinability of difficult-to-cut alloys. The ultrasonic tool post that can be mounted on commercial lathes uses standard interchangeable cutting tools. A prototype ultrasonic boring bar currently in experimental production use is described. Data and photographs representative of machining aluminum and steel are presented. A fluted twist drill is a poor torsional load-carrying structure, and thus it is significant that power can be delivered to the cutting edges of a twist drill via the vibratory mode to the end that static thrust and mechanical torque are reduced, which is especially significant where drill breakage is a problem. Metal removal rates are increased, chip expulsion is facilitated, and the usual four-six dianneters of depth can be increased without drill withdrawal. Ultrasonic twist drilling data are provided for hole diameters up to $\frac{9}{16}$-in. in several materials. Photographs of ultrasonic machining and drilling systems for industrial equipment are presented.

\section{1:50}

L9. Measurement of Acoustic Powers in the MicrowattMilliwatt Range. J. A. Rooney, Physics Department, Universily of Vermont, Burlington, Vermont 05401.-The recent in- crease in the use of ultrasonics in medical diagnosis has led to the need for methods to measure very low acoustic power outputs in the megahertz frequency range. A system similar to that described by Kossoff [J. Acoust. Soc. Amer. 38, 880-881 (1965)] has been developed which uses a Cahn electrobalance to measure acoustic radiation force on an absorbing target. At present the system can easily detect power outputs of $30 \mu \mathrm{W}$. Sources of error and how they can be eliminated in such measurements will be discussed. Recently questions have been raised about the validity of the classical theoretical expression for Langevin radiation pressure applicable to the case of a plane traveling wave impinging on an absorbing target in an open vessel. However, a critical review of the theory using a formulation based on Euler's momentum theorem leads one to the conclusion that the Langevin radiation pressure is just equal to the classical value of the energy density even when nonlinearities of the propagation medium and distortion of waveform are considered. Experimental tests of this theory are in progress. [This work was supported by the HAS Project at the University of Vermont, and by the National lnstitutes of Health.]

\section{2:05}

L10. Pressure Fluctuations Resulting from the Interaction between a Shock Wave and a Turbulent Boundary Layer. E. Grande, Wyle Laboratories, El Segundo, California 90245 . An experimental investigation has been conducted to determine the pressure fluctuations in the external fow resulting from the interaction between an oblique shock wave and a 
turbulent boundary layer. The flow perturbations were measured and interpreted by means of supersonic hot wire techniques. It was found that attached and weakly separated interactions generate weak fluctuating disturbances. However, as the incident shock strength was increased to generate a strongly separated interaction, the fuctuation levels were correspondingly increased. Comparison with Ribner's shockturbulence interaction theory showed that his interaction model is not directly applicable to this particular interaction phenomenon. The experimental results show that the pressure fluctuations in the external flow are mainly generated by the oscillating separation shock. A qualitative analysis, based partly on Ribner's theory, suggests that these shock oscillations are caused by nearfield pressure fluctuations generated by the interaction between the incident shock and the boundary layer, amplified in the separation interaction region, and fed upstream within the boundary layer to the separation shock.

* The study was conducted at the University of Washington, Seattle, Washington.

WEDNESDAY, 19 APRIL 1972

GOVERNOR'S ROOM, 9:00 A.M.

Session M. Physics of Sound in the Sea I : Propagation

Alan Sykes, Chairman

Office of Naval Research, Arlington, Virginia 22217

Contributed Papers (12 minutes)

$9: 15$

M1. Propagation Loss as Predicted by Modified Ray Theory. J. A. DAvrs, Woods Hole Oceanographic Institution, Woods Hole, Massachusetts 02543. - Modified ray theory predicts a frequency-dependent distortion of the sound field which is obtained by ordinary ray theory [J. A. Davis and E. L. Murphy, J. Acoust. Soc. Amer. 50, 101 (1970)]. One qualitative consequence of this distortion is the introduction of a caustic in problems where ray theory predicts a shadow boundary. In order to test this approximate theory and determine the significance of such effects, a computer program has been developed which computes propagation loss for a variety of problems which, while simple, are pertinent to underwater sound propagation. The results of a numerical investigation of propagation loss versus range are presented as a function of frequency, material properties, and boundary conditions (unbounded, pressure release surface, rigid boundary). Comparisons are made with ray theory and available exact solutions. The accuracy of the method in determining propagation loss in the ray-theory shadow zone is strongly dependent upon the treatment of the caustic. The simplest (and most practical) treatment leads to a propagation loss that follows the exact answer to within $2 \mathrm{~dB}$ for at least two decades into the shadow region.

$$
\text { 9:45 }
$$

M2. Parameterless Intensity Formula from the Eikonal Function. D. H. Woon and ANDrew A. Lesick, New London Laboratory, Naval Underwater Systems Center, New London, Connecticut 06320.-Let $y$ and 2 denote cylindrical coordinates and let sound speed be a function $c(z)$ of $z$ alone. Let $r=0$ and $z=z_{0}$ be the coordinates of a point harmonic source. If we can find a solution $t\left(r, z, z_{0}\right)$ of the eikonal equation $(\nabla t)^{2}=c^{-2}$, then the ray acoustics approximation to the intensity of the sound field can be expressed as

$$
10 \log _{10}\left|c c_{0} t_{r} t_{r r}\left(1-c^{2} t_{r}^{2}\right)^{-1}\left(1-c_{0}^{2} t_{r}^{2}\right)^{-1 / r}\right| \text {, }
$$

where $t_{r}$ and $t_{r r}$ are partial derivatives of $t$ with respect to $r$ and $c_{0}$ is $c\left(z_{0}\right)$. Notice that the above expression for intensity is an explicit function of $r$ and $z$ without any ray parameters. The level curves of $t$ are, of course, wavefronts. Differentiating the eikonal equation with respect to $r$ shows that the level curves of $t_{r}$ are rays because they are everywhere orthogonal to the wavefronts. Rays, wavefronts, and intensities are found for three examples, $c=\cosh z, \varepsilon=1+z$, and $\varepsilon=e^{3}$, by the above results and explicit formulas for $t\left(r, z, z_{0}\right)$ such as

$$
\cos \ell=\left(\cos r+\sinh z \sinh z_{0}\right) /\left(\cosh z \cosh z_{0}\right),
$$

when $c=\cosh z$, an unpublished result of G. S. Egeland.

\section{0:00}

M3. Some Curious Analytical Ray Paths for Some Interesting Velocity Profiles in Geometrical Acoustics. A. Barnes, Department of Mathematics, Drexel University, Philadelphia, Pennsylvania, And L. P. Solomon, Tetra Tech, Inc., Arlington, Virginia.--Geometrical acoustics problems in oceanic applications are solved using complicated computer codes. This paper presents some cases that may be used to verify these codes. The velocity profiles are depth dependent, range dependent, and depth and range dependent. The ray path solutions, as well as the velocity profiles, are all expressed in terms of analytical, closed-form, or, at worst, tabulated functions.

\section{$10: 15$}

M4. Monte Carlo Applications to Acoustical Field Solutions. J. K. Haviland and B. D. Thanedar, Department of Aerospace Engineering, University of Virginia, Charlottesville, Virginia 22903. - The Monte Carlo technique is proposed for the determination of the acoustical pressure-time history at chosen points in a partial enclosure, the central idea of this technique being the tracing of the rays. A statistical model is formulated and an algorithm for pressure is created, the confirmity of which is examined critically by different approaches and is shown to give the known results. The concepts that are developed are applied to the determination of the transient field due to a sound source in a homogeneous medium in a rectangular enclosure with perfect reflecting walls, and the results are compared with those presented by Mintzer based on the Laplace transform approach, as well as with a normalmode solution. In contrast with these, the Monte Carlo approach is not restricted to the case of perfect reflecting walls, since absorptive walls can be handled. Possible future developments are indicated that would, it is believed, make the Monte Carlo method a valuable tool when boundary condi- 


\section{RD MEETING - ACOUSTICAL SOCIETY OF AMERICA}

tions are complex or when the medium is inhomogeneous. [Aided by NASA grant.]

\section{$10: 30$}

M5. Transmission into a Basin Having a Bigradient SoundSpeed Profile. P. W. Smith, Jr., And JoIE P. Jones, Bolt Beranek and Nezman Inc., Cambridge, Massachusells 02138.A basin is idealized as a flat-bottom region of deeper water surrounded by a gentle, constant-slope transition to a flatbottom region of shallower water ("shelf"). The speed profile is taken to have a constant negative gradient above a fixed axis depth and a constant positive gradient below, both independent of horizontal position. Analytical estimates for averaged sound transmission loss between a point in the basin and a point at variable range have been formulated from ray theory with lossy specular reflection from the surface and bottom. The approach is an adaptation to the bigradient case of earlier work [P. W. Smith, Jr., J. Acoust. Soc. Amer. 49, 96(A) (1971)]. The loss estimate can be very sensitive to frequency, the depth of the point in the basin, and range, as the variable point moves up the slope and over the shelf. A physical interpretation of this behavior, the dominant parameters, and comparisons with data are discussed. [Study supported by Office of . Vaval Research.]

\section{$10: 45$}

M6. The Integral Solution of the Sound Field in a Multilayered Liquid-Solid Half-Space with a Liquid Bottom. Henry W. Kutschale, Lamonl-Doherty Geological Observatory of Columbia University, Palisades, New York 10964.The integral solution of the wave equation is derived by matrix methods for point sources of harmonic waves in a liquid layer of a multilayered half space of interbedded liquid and solid layers. The solution is in a form convenient for numerical computation on a high-speed digital computer. The integral over wavenumber has singularities in the integrand for guided propagation and is conveniently transformed into the complex plane. By a suitable choice of contours, complex poles are displaced to an unused sheet of the two-leaved Riemann surface, and the integral solution for the multilayered system reduces to a sum of normal modes plus the sum of two integrals, one along the real axis and the other along the imaginary axis. These integrals are evaluated by a Gaussian quadrature formula. Numerical computations are presented for propagation in the ice covered Arctic Ocean.

\section{1:00}

M7. Effect of an Ice Layer on Arctic SOFAR Propagation. Henry W. Kutschale, Lamont-Doherty Geological Observatory of Columbia University, Palisades, New York 10964.The effect of an ice layer on Arctic SOFAR propagation is investigated from the normal-mode solution of the sound field in multilayered media. For an ice layer $3 \mathrm{~m}$ thick, typical of the central Arctic Ocean, the effect on pressure amplitudes at depth and dispersion is small, but even this thin ice sheet causes a large change in particle motions near the surface. Waves are elliptically polarized in the ice in the plane of propagation. Particle motion is retrograde elliptical at the surface and prograde elliptical at the bottom of the ice. This orbital motion is similar to that of flexural waves in the ice. Flexural waves generated by large-scale ice movements at the boundaries of floes are the principal background noise in the ice. It is significant that a node of horizontal particle motion of ten does not occur at the same depth in the ice for both hydroacoustic waves and flexural waves. This effect may be useful to improve the signal-to-noise ratio when horizontal component geophones are used as listening devices.

\section{$11: 15$}

M8. Acoustic Propagation in a Wedge. A. A. Hudimac, $S R A$, Inc., Rockville, Maryland 20854, AND D. BRADLEY, DOL, White Oak, Silver Spring, Maryland 20010.-The wavefield due to a point source in a fluid in a wedge cannot be specified solely by the method of images (except for a wedge angle equal to a submultiple of $\pi$ ). Various methods have been developed by which a finite number of real images are augmented by an integral term. In the case of Bromwich theory, the theorem of Plemij and Sokhotskikh is used to prove the correctness of the added integral. The connection is made between this formulation and our earlier work, in which the wavefield is developed in terms of a Riemann surface fundamental solution. Specifically, the nature of the field in the ensonified and diffracted region is given, and the integralterm contribution is discussed in terms of virtual sources.

\section{1:30}

M9. Statistics of Distant Shipping Noise. I. DYer AND J. C. O'ConNor, Department of Ocean Engineering, MIT, Cambridge, Massachuselts 02139. - In an earlier paper by Dyer, a rudimentary analytical model was proposed for distant shipping noise in the ocean. This model has been extended to reflect actual ocean conditions betier. With this extension, the dependence of statistical parameters on the disposition of ships at sea and on the observational beamwidth and bandwidth may be more readily understood. Predictions of the newer model compare favorably with measurements and are believed to be valid in the frequency range within which the noise of distant shipping dominates wind-generated sea surface noise or noise of other mechanisms. The dependence on depth and the temporal correlation of the noise envelope are not explicitly considered in the model, but will be discussed.

\section{$11: 45$}

M10. The Nature of Sound Fluctuation in Continuous, Statistically Isotropic Media. J. A. Neunert, Naval Underseo Research and Development Center, Pasadena, California 91107. -The stochastic Eulerian-Lagrangian procedure, which was reported earlier, is reviewed heuristically and applied to steady-state sound propagation from a small, collimated acoustic source to an omnidirectional point receiver imbedded in an infinite, continuous, statistically isotropic medium (such as a turbulent fluid.) Part I relates the analysis to ray-trace type relations and emphasizes the need to use Eq. 14 of "Approach to an Eulerian-Lagrangian Problem for Sound Propagation in Continuous Stochastic Media" [J. A. Neubert, J. Math. Phys. 12, 1030-1034 (1971)]. The physical problem is then clearly stated and illustrated.

\section{2:00}

M11. The Nature of Sound Fluctuation in Continuous, Statistically Isotropic Media. J. A. NeUneRT, Naval Undersea Research and Development Center, Pasadena, California 91107 .An analytic procedure is developed for obtaining the Lagrangian measure function $B(\mathcal{X}, \xi \mid s)$ from its characteristic function $\phi(k, \xi \mid s)$ for stochastic-Fermat media. This permits the calculation of the (Eulerian) ensemble average of the pressure wave, $(p(\mathcal{X})\rangle$, as well as $\left\langle|p|^{2}\right\rangle$, via a central limit theorem for stochastic Lagrangian functionals. This results in a coefficient of intensity variation $V$ that evinces a frequencydependent phase-dominance region and a frequency-independent amplitude-dominance region. The methods employed in this study are new to the problem of sound propagation through continuous stochastic media and avoid three common difficulties: (1) range limitations due to cumulative phase effects; (2) discrete scattering associations; and (3) restriction to an Eulerian path. 


\title{
Session N. Engineering Acoustics II : Horns and Horn Loudspeakers
}

\author{
HARRY B. Miller, Chairman \\ Naval Underwater Systems Center, New London, Connecticut 06320
}

\section{Invited Papers (30 minutes)}

\begin{abstract}
9:00
N1. Loudspeaker Horn Theorg-A Review. V. Salmon, Stanford Research Institute, Menlo Park, California 94025.-The theoretical and applied contributions of Webster, Hanna, Bostwick, Olson, Salmon, Mawardi, and Klipsch (among others) will be reviewed, using the impedance concept as a unifying principle. The characteristics of the complete audio source-driver-horn-radiation field system will be examined from the points of view of steady-state and transient response.
\end{abstract}

$$
\text { 9:30 }
$$

N2. Historical Notes on the Use of Horns for Sound Reproduction. John K. Hilliard, Ramberg \&o Lowrey, Santa Ana, California 92706. - This paper will start with the early application of horns for sound reenforcement beginning around 1920 and describing the changes and types of driver units used. The introduction of sound to motion pictures required intensive applications of efficient drivers and horns because of limited amplifier power. The evolution of theater and high-power sound-projection systems will be described and how these changes have provided better distribution, increasedfrequency range, and lower distortion.

\section{0:00}

N3. Sound Propagation in Horns. O. K. MAward, School of Engineering, Case Western Reserve University, Cleveland, Ohio 44106.-A generalized theory is developed for the theory of propagation of sound waves in horns of semiinfinite length. A few approximate treatments are described to derive in closed form the acoustic impedance of horns. Some specific examples will be given for horns whose contour is described by analy tic functions.

\section{$10: 30$}

N4. Impedance-Transforming Wave Systems with Spatially Varying Density or Elasticity. E. A. G. Shaw, Division of Physics, National Research Council of Canada, Ottawa, K1A OS1.-Acoustic impedance can be transformed either with an acoustic horn that controls the variation of wavefront area as the wave progresses, or by transmitting the waves through a medium in which there is a gradient of density or elasticity. Analysis shows that for plane waves a medium in which density varies as $(B+m x)^{-9}$ or elastic modulus as $(B-m x)^{2}$, where $x$ is distance and $B$ and $m$ are constants, is analogous to the ideal exponential horn. Furthermore, radial variations of density and elasticity can readily be found which transform the behavior of sectoral and conical horns into the acoustic equivalents of exponential horns. Since the wave equation is separable in both cylindrical and spherical coordinates, these solutions are exact. In practice, the effective density of a fluid medium can be increased by introducing suitable arrays of obstacles, and the bulk modulus of a liquid decreased by compliant inclusions. The relationships between array size, bandwidth, and damping are discussed, and three artificial fluid structures are suggested.

$$
\text { 11:00 }
$$

N5. Brass Instrument Design and the Theory of Horns. A. H. BENADE, Case Western Reserve University. Cleveland, Ohio 44106.- Engineering the design of horns has been chiefly directed toward transducer applications, where freedom from resonances and a low cutoff frequency are desirable. In the musical horns, easy oscillation and good tuning both call for a high cutoff frequency and accurately placed resonances. The radiation load must not be more than a perturbation at playing frequencies. Horns of rapid flare are required, for which plane-wave theory is inadequate. The "reduced" pressure wave function $\psi(z)(=p S t)$ is mathematically and conceptually convenient, where $S(z)$ is the area of the spherical wavef ront at the point $z$ along the horn axis. The "reduced" spherical wave equation $\psi^{\prime \prime}+\left[k^{2}-U(z)\right] \psi=0$ (studied by Jansson) is tractable, the horn function $U$ being easily visualized in terms of the transverse and longitudinal curvatures of the horn. Exact solution of the equation is not always required, since useful estimates of $U$ can be made by inspection, whence calculations related to phase integrals, plus perturbation theory permit excellent approximations to the resonance frequencies. Different perturbation methods are useful along the horn depending on local flare. Preexisting horns may be diagnosed and corrected on the basis of laboratory data or "musicians' experiments." Practical examples will be discussed. 


\title{
Contributed Paper
}

$11: 30$

N6. Horn Theory and the Phonograph. Percy Wilson, Percy Wilson and Partners, (56 Slaunion Road), Oxford $\mathrm{OXX}_{3}$ 7TP, England, and Goeffrey L. Wilson, The Pennsylvania State University, University Park, Pennsylvania 16802.-This paper describes the development of horn theory from Ray- leigh, through Webster, Hanna and Slepian, Percy Wilson, P. B. Flanders, and Paul Voigt up to the comprehensive analysis by Leo Beranek. It includes an account of some early experiments by the senior author on methods of folding horns. A description is also given of a recent acoustic phonograph with a fabulous response down to $30 \mathrm{~Hz}$ thanks to loading by an external exponential horn that is curved rather than folded.

\section{Session O. Machine Noise II : Noise Measurement of Machines as Installed}

\author{
O. L. Angevine, Jr., Chairman \\ Anderson and Angevine, Inc., East Aurora, Nerv York 14052
}

\begin{abstract}
Discussion of individual papers will be suspended until all papers have been delivered when a discussion of the complete session will be lead by $T$. J. Schultz.
\end{abstract}

\section{Invited Papers}

01. Noise Measurement Standards for Machines in situ. WilliaM W. Lang, IBM Acoustics Laboratory, Poughkeepsie, New York 12602. - Two classes of documents prescribe standards for noise measurements on machines and stationary equipment in the field. Fundamental methods of measurement are described in documents prepared by American National Standards Committee S1 for which the Acoustical Society of America serves as the Secretariat and by Technical Committee 43 of the International Organization for Standardization (ISO). Test codes applicable to specific types of equipment are prepared in the United States by engineering societies and trade associations. These two classes of documents should be mutually supportive and interdependent, but such is not always the case. The requirements of existing standards on noise measurements in situ are summarized, the shortcomings of these documents are described, and the needs for new standards are reviewed. An estimate is given of the degree to which current standardization activities are satisfying these needs.

O2. Sound Fields near Arrays of Noise Sources. V. Salmon, Stanford Research Instilute, Menlo Park, California 94025.- When sound from different portions of an extended radiator is essentially noncorrelated, the source is said to be spatially incoherent (SI). Such sources can be characterized by power radiated per unit length, area, or volume. The total field is then obtained by power addition, using the inverse square law. The consequences of this simplifying assumption for measuring machine noise will be reviewed in the light of work by Salmon, Maekawa, Embleton, Shaw, and others.

03. Nearfield Measurements and Sound Power. O. L. Angevine, JR., Anderson and Angevine, Inc., East Aurora, New York 14052. - The measurement of noise generated by large machines in ordinary semireverberant rooms can only be done very close to the machine at distances which appear to be well within the nearfield of the machine considered as a single large noise source. But large machines are usually composed of a number of smaller incoherent noise sources. Arrays of such sources show the farfield beginning at distances comparable to the measuring points at $1 \mathrm{~m}$ from the machine used in the CAGI standard, or the prescribed path of ISO Recommendation 1680. Such measurements corrected to a uniform radius are used to determine total sound power. The results agree well with sound power calculated for the same machine from farfield measurements.

O4. Errors in the Measurement of Machine Noise. Gerharn HügnER, Laboralorium fur Maschenengeräusche, Siemens AG, Dynamowerk, 1 Berlin 19, West Germany.-Even under free-field conditions, there are three types of errors in the measurement of machine noise: (1) the nearfield error; (2) the error caused by using a limited number of measuring points; and (3) the error in measuring sound pressure at each point. The magnitude of these errors for measurements made in ordinary rooms following the procedure of ISO Recommendation 1680 is discussed.

O5. Steady-State Noise Characteristics of Semireverberant Spaces. Douglas J. InGalls, Carlyle Compressor Company, Syracuse, New York 13201.-The use of a steady-state reference sound source, such as the $\mathrm{Ilg}$ Reference Sound Source, for determining the acoustic nature of semireverberant spaces is explored with emphasis on what precautions should be observed if useful engineering results are to be obtained. For reasonable acoustic environments, the steady-state method can also be used 
to estimate the room absorption or, by the substitution technique, the sound power of an unknown source.

O6. Practical Aspects of Machine Noise Measurement as Installed. L. H. BeLl AND Harold R. M ULL, Bell and Associates, Willon, Connecticut 06897.-Many industrial manufacturing and processing companies, at the urging of plant engineers and safety directors, are requiring maximum noise level guarantees for purchased machines. Since "as installed" measurements form the basis of acceptance and provide plant engineers the only information available to assess noise impact, many questions are being asked about the value and practical aspects of these measurements. Presented in this paper are some practical aspects that must be considered in (1) choice of instrumentation; (2) treating unwanted reflecting surfaces and background noise; (3) dealing with pure tones and nonsteady noise levels; and (4) assessment of probable range of errors. The discussion will also include some practical applications of "as installed" measurements.

O7. Persisting Questions in Steady-State Measurement of Noise Power and Sound Absorption. T. J. Schultz, Bolt Beranek and Nezman Inc., Cambridge, Massachusetts 02138.-Most steady-state methods for measuring the noise power of equipment and the sound absorption in a room depend on knowing the sound power output of the standard source. But the output of any sound source depends in principle on the radiation impedance presented to it, and this impedance is likely to change with the location of the source, as well as with changes in the amount and distribution of absorptive material in the room. Moreover, no generally accepted method is available for measuring the soundpower output directly. For these reasons, the validity of steady-state methods has been under suspicion for some time. This paper summarizes recent progress in making steady-state methods respectable but calls attention to some remaining problems.

WedNESDAy, 19 ApriL 1972

EMBassy RoOM, 9:00 A.M.

\title{
Session P. Psychological Acoustics I : Signal Processing
}

\author{
BARRY Leshowitz, Chairman
}

\author{
Arizona State University, Tempe, Arizona 85281
}

\section{Contributed Papers (12 minutes)}

9:00

P1. Human Cochlear Partition Stiffness. P. Allaire, S. Raynor, AND M. BILlone, Mechanical Engineering Department, Northwestern University, Evanston, Illinois 60201.-A mathematical model of the human cochlear partition is developed to investigate in depth the partition's stiffness. To date, no experiment has been performed on the partition to determine the difference between the dynamic stiffness and the statically measured stiffness. Nonlinearities observed in the partition's dynamic motions and dynamic stiffness measurements on bone and muscle tissue indicate that previous studies of the cochlear partition's stiffness may not be conclusive. In this study, the cochlear partition is modeled by a series of composite beams whose stiffness is due to linear bending rigidity at normal amplitudes of motion. At larger amplitudes (in the nonlinear range near the threshold of pain), the model's stiffness is increased due to the nonlinear stretching of the basilar membrane. The nonlinear model may account for the observed nonlinearity. The model also includes the increase in stiffness, above the static stiffness, due to dynamic loading as observed in the studies of bone and muscle tissue. The dynamic cochlear partition stiffness predicted by the model varies approximately 100 fold from base to apex. Except near the stapes, the model's stiffness in the linear range at $100 \mathrm{~Hz}$ is approximately the same as the statically measured stiffness. At $10000 \mathrm{~Hz}$ the model's stiffness in the linear range is nearly double the statically measured stiffness.

\section{$9: 15$}

P2. New Boundary Conditions and Results for the PetersonBogert Model of the Cochlea. C. D. Geisler AND A. E. HuB-
BARD, Electrical Engineering Department and Laboratory of Neurophysiology, University of Wisconsin, Madison, Wisconsin 53706.-The Peterson-Bogert model of the cochlea [J. Acoust. Soc. Amer. 22, 369-381 (1950)] specifies, as one of its boundary conditions, zero pressure at the round window. This specification is equivalent to eliminating the round-window membrane. We have changed this boundary condition to include the round-window membrane, representing it with an acoustic compliance. With the new boundary condition, the equation for $p_{+}(x)$ requires a new solution, and a new value of $p_{-}(x)$ at $x=0$ is needed. The new solution for $p_{+}(x)$ and the new value of $p_{-}(0)$ are given, for sinusoidal stimulation, as a function of frequency. The latter value is needed as the input to the Peterson-Bogert type of model of the basilar membrane. The model's input impedance as well as the relative displacement between the oval and round windows are also given. These relationships are compared with experimental findings.

\section{$9: 30$}

P3. Response of the Primary Auditory Neuron to Human Speech. Allan J. Goldstein and J. Rrland Mundie, 6570 Aerospace Medical Research Laboratory, I'right-Patterson $A F B$, Daylon, Ohio 45433.-A previous report [81st Meeting Acoust. Soc. Amer., J. Acoust. Soc. Amer. 50, 83 (A) (1971)] documented that pulse occurrence histograms from primary auditory neurons of guinea pigs followed the time history of the bidirectional displacement patterns of the basilar membrane predicted by an electronic "analog cochlea" model. The basilar membrane describes a surface when stimulated by an auditory signal. This surface represents the point by point displacement of the basilar membrane along its length 
as a function of time. This paper presents data from many neurons distributed (on the basis of characteristic frequency) along the length of the basilar membrane. The data demonstrate that the temporal and spatial details of the complex surfaces of human speech sounds are preserved in the pulse occurrence histograms up to the temporal resolving power of our computer system $(200 \mu \mathrm{sec})$. At low stimulus intensity, only those features that correspond to large displacements are seen in the pulse occurrence histogram; as stimulus intensity is progressively increased, features corresponding to smaller amplitude displacements progressively appear. There is not a linear relationship between magnitude of displacement and magnitude of neural response. Although the data collected to date have been analyzed on the basis of displacement patterns, we are unable to exclude velocity of the basilar membrane as the input to the primary neuron.

\section{$9: 45$}

P4. A Model for Residue Pitch. Ian B. Thomas and A. Ravindran, Electrical Engineering Department, University of Massachusetts, Amherst, Massachusetts 01002.-We have developed a model of pitch perception that accurately predicts all subjective (residue) pitches measured in the experiments of Schouten, Ritsma, and Cardozo [J. Acoust. Soc. Amer. 34, 1418-1424 (1962)]. The primary assumptions of the model are (1) that the transfer function relating amplitude of basilar membrane displacement at a particular membrane location to acoustic pressure external to the ear represents a bandpass filter of low selectivity and frequency-dependent phase change; (2) that the pattern of axis crossing intervals of the signal representing basilar membrane displacement at any membrane location is neurologically encoded and analyzed; (3) that there is a mechanism capable of detecting a variation of approximately $0.3 \%$ in the duration of axis crossing intervals for the frequency range considered. Prediction of residue pitches requires no assumption of nonlinear distortion anywhere in the filtering process, nor does it require the presence of any amplitude encoding mechanism such as an envelope detector. The model also predicts perception of the difference tone without any appeal to nonlinear processes. Prediction of more complex combination tones does require the assumption of nonlinear distortion in the filtering process. The model gives at least qualitatively satisfactory explanations for other auditory phenomena such as the critical band and masking. [Work supported by NIH.]

\section{0:00}

P5. Rippled Noise Investigations of Lateral Inhibition in the Ear. L. L. FETH, Deparlment of Speech and Hearing Sciences, University of Southern Mississippi, Hatliesburg, Mississippi 39401, D. M. GREEN, Department of Psychology, University of California, San Diego, La Jolla, California 92037, AND W. A. Yost, Communication Sciences Laboratory, Universily of Florida, Gainsville, Florida 32601.-Linear spatial analysis techniques have shown marked success in recent applications to vision. Determination of observers' modulation transfer functions (MTF) for sinusoidal intensity gradients has led to acceptable explanations for Mach bands and other contrastenhancement phenomena. Fourier transformation of the MTF produces a spread-of-excitation function which shows a central excitatory area with an inhibitory surround. We have conducted analogous experiments in audition using wide-band noises with sinusoidally rippled spectra. Using a 2 IFC tracking paradigm, listeners were asked to discriminate between rippled- and Hat-spectrum noise bursts. Plots of ripple sensitivity as a function of ripple (spatial) frequency are analogous to the visual MTF. Fourier transforms of these functions do not exhibit the inhibitory surround, but this is probably due to a pitch cue provided by the rippled noise. [This research was supported by the National Institutes of
Health, U. S. Public Health Service, Department of Health, Education, and Welfare.]

\section{$10: 15$}

P6. Estimation of the Integration Time-Constant in Auditory Receptor Units. E. J. KLETSKY AND J. M. STEnGREvics, Laboratory of Sensary Communication, Syracuse Universily, Syracuse, New York 13210.-Period histograms obtained from eight-nerve units of monkey by Brugge et al. [J. Neurophysiol. 32, 386 (1969)] show a high correlation with positivegoing excursions of the stimulus waveform. A more detailed examination reveals that the histograms decay more slowly than the stimulus, suggesting a temporal integration in the receptor unit. We have estimated the value of the time constant to be between 0.1 and $0.2 \mathrm{msec}$ by matching eighthnerve period histograms with those generated by means of an analog model of the auditory receptor and first-order neuron. These values are consistent with the maximum frequency at which substantial time locking is observed physiologically. The model assumes the following sequence of events. Linear amplitude summation of stimulus and intrinsic noise is followed by half-wave rectification and integration with a time constant of about $0.15 \mathrm{msec}$. The resulting signal, a receptor potential, controls the average rate at which packets of transmitter substance are released to form EPSP in the first-order neuron. The times of packet release are assumed to be random and follow a Poisson distribution. An electronic implementation of the model was constructed and used with a previously developed neuron model to generate data for these studies.

$$
\text { 10:30 }
$$

P7. On Loudness as a Function of Tone Duration. J. J. Zwislocki AND W. G. Sokolich, Laboratory of Sensory Communication, Syracuse University, Syracuse, New York 13210.-According to the prevailing evidence, the loudness level of short tone bursts increases with their duration at a rate of about $10 \mathrm{~dB} /$ decade. It is not clear, however, whether the data refer to total loudness or to instantaneous loudness reached at a particular point in time. In order to answer the question, listeners were instructed to respond to instantaneous loudness at the end of tone bursts in one series of experiments and to total loudness in another. They had to adjust the loudness level of a 10-msec comparison burst accordingly. Not all listeners could change their criterion, but those who could showed that instantaneous loudness depends little on tone duration, whereas total loudness increases at a rate of almost $10 \mathrm{~dB} /$ decade up to a duration of at least $500 \mathrm{msec}$. These results are consistent with our findings on temporal loudness effects in pairs of short sound bursts. Previous results according to which loudness increases up to a duration of about 80 msec may be explained by the listeners' tendency to respond to total loudness at very short durations and to instantaneous loudness at longer ones.

\section{$10: 45$}

P8. On Loudness Enhancement of a Tone Burst by a Preceding Tone Burst. W. G. Sokolich AND J. J. Zwislocki, Laboratary of Sensory Communication, Syracuse University, Syracuse, New York 13210.- According to preceding experiments, loudness effects in pairs of tone bursts are controlled by two processes, loudness enhancement and loudness summation. This paper deals with the effects of intensity, time, and frequency differences between the bursts on the loudness enhancement of the second burst. Listeners were asked to match the loudness of a third (comparison) burst to the loudness of the second burst of a monotic burst pair. All three bursts had a duration of $10 \mathrm{msec}$, and the second and third bursts were at $1000 \mathrm{~Hz}$. The intensity of the first burst was either $+20,0$, or $-20 \mathrm{~dB} r e$ the intensity of the second burst, which was always 
at a sensation level of $40 \mathrm{~dB}$. The interval between the onsets of the first and second bursts was varied stepwise between 10 and $500 \mathrm{msec}$, and the frequency of the first burst, between 200 and $4000 \mathrm{~Hz}$. The results indicate a loudness enhancement that increases with the intensity of the first burst and decreases as the time interval and the frequency difference between the bursts in the burst pair increase. When the first burst was at an intensity level of $-20 \mathrm{~dB}$, a loudness decrement occurred.

\section{1:00}

P9. Neural Phase Locking to Combination Tones Predicted by Simple Transducer Models. JuLrus L. Goldstern, Research Laboratory of Electronics, MIT, Cambridge, Massachusetts 02139.- Conceptual distinctions can be made between nonlinearities arising in stimulus processing prior to transduction and those arising during transduction to neural events. The class of model transducers is considered for which a memoryless nonlinear transformation generates from the processed stimulus $X$ a non negative signal $R(X)$, which then in direct proportion determines the instantaneous probability of a neural event. For $X$ comprising the incoherent tones $a \cos \omega_{1} t$ $+b \cos \omega_{2} t$, the expected period histogram referenced in cosine phase to the combination frequency $p \omega_{1}+q \omega_{2}$, for $p$ and $q$ integral, nonzero and possessing no common integral factor greater than one, is proportional to

$$
\begin{aligned}
P\left(p \omega_{1}+q \omega_{2}, \theta\right) & \\
& =\frac{1}{2 \pi} \int_{0}^{2 \pi} R\{a \cos q \phi+b \cos (p \phi-\theta / q)\} d \phi \cdots .
\end{aligned}
$$

For $R(X)=\exp (X)$, the expected histogram for any integral $p$ and $q$ is proportional to

$$
\begin{aligned}
P\left(p \omega_{1}+q \omega_{2}, \theta\right)=I_{0}(a) & I_{0}(b) \\
& +2 \sum_{n=1}^{\infty} I_{n p}(a) I_{n q}(b) \cos n \theta \cdots,
\end{aligned}
$$

where $I_{m}(z)$ is the $m$ th-order modified Bessel function of the first kind. Application of this theory to auditory-nerve data clarifies some aspects of nonlinear stimulus processing prior to transduction. [Work supported by a grant from the $\mathrm{Na}$ tional Institutes of Health.]

\section{1:15}

P10. Detection of a Missing Harmonic in a Continuous or Pulsed Harmonic Complex. Neal F. Viemeister and David M. GReEN, University of California at San Diego, La Jolla, California 92037. - The masker was a complex of harmonically related sinusoids with the $m$ th harmonic missing. The signal was the $m$ th harmonic and its threshold was determined with the masker either continuously presented or pulsed, i.e., gated simultaneously with the signal. The missing harmonic is always more detectable with the continuous rather than the pulsed masker. Differences in thresholds between the two conditions range from 2 to $25 \mathrm{~dB}$. With a continuous masker the phase of the signal infuences the threshold by as much as 15
dB. No such phase effects were observed with pulsed maskers. The difference between continuous and pulse thresholds also depends on signal frequency, number and spacing of the components, relative phases of the components, and masker level. [This research was supported by the National Institutes of Health, Public Health Service, U. S. Department of Health, Education, and Welfare; and by a Special Traineeship from NINDS.]

$$
11: 30
$$

P11. The Critical Masking Interval. M. J. Penner, Psychology Department, University of California, San Diego 92037, Charles E. Robinson, Neurophysiology-Biophysics Research Laboratories, Veterans Administration Hospital, Martinez, California 94553, AND DAvid M. GREen, Psychology Department, University of California, San Diego 92037.-We investigated the concept of a critical masking interval, the time period during which noise is effective in masking a brief signal. The signal was a $100-\mu$ sec click temporally placed in the center of a gated-noise masker. Both the click and the noise were lowpass filtered so that the power spectra of signal and noise have similar shape. The duration of the noise masker was varied from $100 \mu \mathrm{sec}$ to $100 \mathrm{msec}$ and two noise levels were employed. Like the estimate of the critical bandwidth, the estimate of the critical masking interval can be made in two independent ways. First, for long durations of the noise masker, the ratio of click energy to over-all noise power, adjusted for energy resolution, indicates that the critical masking interval is about $14 \mathrm{msec}$. Second, progressively shortening the duration of the noise masker did not improve detectability until the duration was less than approximately $10 \mathrm{msec}$. The near agreement of these two independent estimates supports our interpretation of the data in terms of the concept of a critical masking interval.

\section{$11: 45$}

P12. Detection of Sinusoids in Noise as a Function of the Signal-to-Noise Ratio on Individual Trials. Howard KaPLAN, Department of Psychology, University of Toronto, Toronto 181, Ontario, Canada.-Current models of the auditory detection of a sinusoid in a noise background propose that the probability that a subject will claim to detect the sinusoid on a particular trial increases with the total energy present in a critical frequency band on that trial. This leads to the counterintuitive prediction that, among a set of true signal-with-noise trials in a single run in which a subject is working with a fixed criterion, it is those trials with the most noise background on which the subject is most likely to detect the signal. An alternative theory is that the subject discriminates signal trials from noise-alone trials on the basis of the contrast, or energy difference, between the largest single component in a band of frequencies and the mean energy of the components. This theory predicts that those trials with the least noise added to a signal are those on which the subject is most likely to detect the signal. Experiments designed to compare these two theories have yielded data consistent with the predictions of the second, or energy contrast, theory.

\section{Session Q. Physiological Acoustics II : Cochlear Physiology}

George Moushegian, Chairman

Callier Hearing and Speech Center, Dallas, Texas 75235

\section{Contributed Papers (12 minutes)}

\section{1:15}

Q1. Some Properties of Response Patterns in the Cochlear Nerve of the Pigeon. M. B. Sachs, R. H. Lewrs, and E. D.
Young, Department of Biomedical Engineering, Johns Hopkins

University School of Medicine, Baltimore, Maryland 21205.Discharges from more than 200 single fibers in the cochlear 
nerve of the pigeon have been recorded with $\mathrm{NaCl}$-filled micropipets. The median rate of spontaneous activity for these fibers is approximately 80 spikes/second, with some fibers discharging at rates higher than 150/second in the absence of controlled acoustic stimuli. Responses to low frequencies (less than $4.0 \mathrm{kHz}$ ) are phase locked. Isorate contours have been measured, taking as response measures both average rate of discharge and number of synchronized spikes per cycle. Characteristic frequencies for the population studied lie in the range $100 \mathrm{~Hz}$ to $6.5 \mathrm{kHz}$. Click-response patterns are similar to those seen in the cat. Two-tone inhibition is also demonstrated. [Supported by the National Institute of Neurological Disease and Stroke and the U. S. Air Force Office of Scientific Research.]

\section{$1: 30$}

Q2. Cochlear Potentials of the Pigeon: Relationships to Age, Serum Cholesterol Level, and Atherosclerosis. JAMES G. McCormick, Robert M. Clayton, and Ivan L. Holleman, JR., Section of Otolaryngology and Department of Pathology, Bowman Gray School of Medicine, Winston-Salem, North Carolina 27103.-The cochlear potentials of different breeds of pigeons were measured. Potentials of old pigeons that developed spontaneous carotid atherosclerotic plaques were generally $15-18 \mathrm{~dB}$ poorer in sensitivity than younger birds of the same breed with little or no evidence of plaques. In another breed of pigeon, resistant to spontaneous atherosclerosis, but susceptible to high-cholesterol-diet-induced coronary atherosclerosis, differences in potentials were less clearly defined; however, both the older birds and younger diet treated birds tended to be less sensitive than young nondiet treated birds.

\section{$1: 45$}

Q3. Effects of Choline Salicylate on the AC Cochlear Potential in Guinea Pigs. D. L. McPherson AND J. M. Miller, Department of Otolaryngology, University of Washington Medical School, Seattle, Weshington 98195.-AC cochlear potentials were recorded from the round window of guinea pigs anesthetized with Nembutal. Following placement of the electrode, the auditory bulla was closed and pure tones were presented via a speaker located $5 \mathrm{~cm}$ from the ear. Intensities were determined with calibrated Brüel \& Kiær probe tubes and condenser microphones at the opening of the external auditory meatus. One microvolt contours examined with a General Radio wave analyzer (3- $\mathrm{Hz}$ bandwidth) across frequency $(0.2$ to $20 \mathrm{kHz}$ ) were found to agree with accepted guinea pig sensitivity curves. Following oral administration of choline salicylate, a rapid and significant decrease in the AC cochlear potential was observed. Following a peak effect (at 3-4 h) and plateau, initial recovery was noted. The effect was drug dose dependent. The effect was frequency specific, with the greatest influence on the middle-frequency range. The maximum amplitude at the point of nonlinearity of the AC cochlear potential was reduced with drug. Also the point of nonlinearity was reached at a higher intensity of tonal stimulation following drug administration. Detailed findings on the cochlear effects of choline salicylate will be described. The interpretation of these effects on generation of the $\mathrm{AC}$ cochlear potential will be discussed. [This research was supported by an NIH grant and by the Deafness Research Foundation.]

\section{2:00}

Q4. Ototoricity of Lead: VDL and AP Changes in Chronically Poisoned Guinea Pigs. Chester Wilpizeski and Wolfgang VOGEL, Depariments of Otalaryngology and Pharmacology, Thomas Jefferson University, Philadelphia, Pennsylvania 19107.-Evidence for lead-induced auditory impairment was obtained by recording VDLs and AP input-output functions for click stimuli presented to guinea pigs having permanently implanted electrodes located in the auditory nerve-cochlear nucleus region. Six animals were fed approximately $1 \mathrm{~g}$ of lead acetate per day mixed with powdered food rations for a 3 month period following weaning (chronic lead poisoning). Six additional animals served initially as a control group but were later injected intraperitoneally daily with $10 \mathrm{mg} / \mathrm{kg}$ of lead acetate in an aqueous solution. Mean VDLs recorded from the chronic group were raised $14 \mathrm{~dB}$ above control values for continuous and interrupted trains of 100 clicks per second. Input-output functions showed a flattening of slope at higher click intensities. Acutely poisoned animals died within 2 weeks without demonstrable shifts or AP changes attributable to lead effects. [Supported in part by USPHS.]

$$
\text { 2:15 }
$$

Q5. Ototoxicity of Ethacrynic Acid Enhanced by Barbiturate Anesthesia. Chester Wilpizeski, Stephen IsaAcson, and Salah Saliman, Department of Otolaryngology, Thomas Jefferson University, Philadelphia, Pennsylvania 19107.VDLs and input-output functions for CM and AP were recorded under waking and anesthetized conditions (35 $\mathrm{mg} / \mathrm{kg} \cdot$ of pentobarbital sodium) from guinea pigs having implanted depth electrodes localized in the chochlear nucleus region or flexible electrodes fixed against the round-window membrane. Following control recordings, animals were injected intraperitoneally with $50 \mathrm{mg} / \mathrm{kg}$ of ethacrynic acid and measurements repeated at 1,2 , and $4 \mathrm{~h}$ post drug. AP VDLs for clicks were elevated $15 \mathrm{~dB}$, and suprathreshold evoked response amplitudes were reduced below normal at low to moderate stimulus intensity levels. CM VDLs for pure tones recorded during barbiturate anesthesia shifted $35 \mathrm{~dB}$ (median for all pure tones) but only $15 \mathrm{~dB}$ under waking conditions. Slopes of CM input-output functions were not altered by ethacrynic acid as were AP amplitude curves; rather, $\mathrm{CM}$ slopes remained invariant and parallel but were depressed compared with control values.

$$
2: 30
$$

Q6. Effect of Potassium Deficiency on Cochlear Potentials and Cation Content in Endolymph. T. KonISHI AND E. KELSEY, Department of Surgery, University of Florida, Gainesville, Florida 32601. - The scala vestibuli and/or scala tympani in guinea pigs were perfused with $\mathrm{K}^{+}$-free modified Ringer's solution. Cochlear microphonics (CM), summating potential (SP), action potential (AP), and endocochlear potential (EP) were recorded from the basal turn of the cochlea before, during, and after the perfusion. Perfusion of both scala vestibuli and tympani with the normal Ringer's did not result in appreciable changes in the cochlear potentials or $\mathrm{Na}$ and $\mathrm{K}$ content in the endolymph. Perfusion of the perilymphatic space with $\mathrm{K}^{+}$-free Ringer's caused significant suppression of EP and AP. The $C M$ was also reduced in magnitude and accompanied by increase of the positive SP. These changes were reversible by washing with normal Ringer's. The measurement of $\mathrm{K}$ and $\mathrm{Na}$ content in the endolymph indicated that the $\mathrm{K}$ deficiency in the perilymph brought about significant increase of $\mathrm{Na}$ content and decrease of $\mathrm{K}$ content. The role of $\mathrm{K}$ ions in the perilymph for the generation of EP is discussed. [Work supported by a Public Health Service grant].

* Also from Communication Sciences Laboratory and Department of Physiology.

$$
2: 45
$$

Q7. Release of Acetylcholine by the Olivocochlear Bundles. P. S. Guth, M. Burton, AND C. H. Norkis, Department of Pharmacology, Tulane University. New Orleans, Louisiana 70112.- The mechanism by which the olivocochlear bundle (OCB) fulfils its presumed feedback inhibitory role is un- 
known. However, acetylcholine (ACh) has received experimental support as the inhibitory mediator of the OCB haircell junction. In the present investigation, the ability of the $\mathrm{OCB}$ to release $\mathrm{ACh}$ upon electrical stimulation was tested. Guinea pigs, anesthetized with pentobarbital and immobilized with gallamine, were prepared for this research by placing recording electrodes on both round windows, stimulating electrodes on the floor of the fourth ventricle, and push-pull perfusion pipet in the scala tympani and scala vestibuli of one cochlea. The cochleas were perfused with artificial perilymph containing eserine. Samples of perfusate were collected before, during, and after OCB stimulation and bioassayed for ACh-like activity on the isolated guinea pig ileum. The authenticity of the ACh-like activity, when present, was verified by hydrolysis by acetylcholinesterase or antagonism by low concentrations of atropine. Concentrations of ACh-like activity detected in the perfusate were of the order of $10^{-11}$ moles/ml. An increase in the ACh-like activity was seen in conjunction with OCB stimulation. [Supported in part by V. A. 71-6.]

Q8. Computer Simulation of Cochlear-Microphonic Potential Augmentation During Intracochlear Perfusion. David $\mathrm{H}$. Moscovitch and Charles A. Laszlo, BioMedical Engineering
Unit and Institule of Ololaryngology, McGill University, Montreal, Canada.-In previous studies, "start-stop" perfusion of scala tympani by artificial solutions produced temporary augmentation of the $C M$ recorded by differential electrodes. The significance of hydrodynamic changes in scala tympani and the differential action of the artificial and natural perilymph on the hair cells in this phenomenon was investigated by computer simulation. In particular, it was determined from fluid-dynamic and experimental considerations that the artificial perilymph perfusate could be spontaneously replaced by natural perilymph at a rate of 0.8 $\mu \mathrm{liter} / \mathrm{min}$. The effect of this replacement was simulated on a computer model of the mechanoacoustical and mechanoelectrical transduction process of the guinea-pig ear [C. $A$. Laszlo, J. Acoust. Soc. Amer. 38, 93(A) (1971)]. After simulated injection of a solution whose action was to suppress the CM generated by individual hair cells, the modeled gross CM was temporarily augmenled by $40 \%$ above the preperfusion control, comparable to that observed in our perfusion experiments. The results indicale that the experimentally observed CM augmentation may be due to partial suppression and spontaneous progressive recovery of the $C M$ generators, resulting in the detection of an apparant rather than a real CM augmentation. [Work supported by the Medical Research Council of Canada.]

Wednesday, 19 April 1972

GOVERNOR's ROOM, $1: 40$ P.M.

\title{
Session R. Nonlinear Acoustics III : Solids and Liquids
}

\author{
R. B. Lindsay, Chairman \\ Brown University, Providence, Rhode Island 02912 \\ Invited Paper (25 minutes)
}

\begin{abstract}
R1. The World of Nonlinearity. R. T. BEyER, Deparlment of Physics, Brown University, Providence, Rhode Island 02912. - As we attempt to describe the physical world more accurately, we find that linear relations among the variables of interest, and linear differential equations become inadequate. For want of a positive term, we cover all such situations with the word nonlinear. In particular, sound propagation in a particular substance will be nonlinear in character whenever the intensity of sound is sufficiently high, or when there are nonlinear terms in the equations describing the medium, or when there are nonlinearities involved in the boundary conditions. The term nonlinearity of the medium may involve the simple relation of the adiabatic equation for an ideal gas, the third-order elastic constants in solids or the characteristics of a vortex or a plasma. Our knowledge of sound propagation can both contribute to and benefit from our knowledge of these nonlinearities.
\end{abstract}

\section{Contributed Papers (12 minutes)}

\section{2:05}

R3. Developments in the Use of Acoustic Shock Pulses in the Study of Elastic Properties of Solids. A. Nakamura, Y. Yasumoto, AND R. TAKEUCh, Instilule of Scientific and Industrial Research, Osaka University, Suila, Osaka, Japan.-A nondestructive method of measurement is being developed for the purpose of investigation of the dynamic behavior of solids, especially concerned with nonlinear elasticity. In this paper, we discuss a method of generation of reproducible acoustic shock pulses in various solids, and also discuss the measurements of the stress-strain relationship using intensive acoustic shock pulses. Results obtained are the following: In aluminum samples, a linear relationship between stress and strain is found experimentally for acoustical pressures up to 130 bar before annealing and also up to 65 bar after annealing. On the other hand, after annealing the specimens, we found a variation of pulse shape and a decrease of peak value compared with the pulse shape before annealing. A qualitative discussion is given of photographs of changes in the shapes of the shock pulses that have propagated through solids.

* Present address: Department of Physics and Astronomy. The University of Tennessee, Knoxville, Tenn. 37916. 


$$
2: 20
$$

R4. Ultrasonic Second-Harmonic Generation in Resonant Nonlinear Elastic Solids. R. D. PETERS, Department of Physics and Astronomy, The University of Mississippi, University, Mississippi 38677. - Second-harmonic and fundamental standing wave amplitudes have been measured to see if nonlinearity parameters (combinations of third-order elastic constants) can be determined from measurements made on resonant samples. Measurements were made on five different materials, all single crystals except for a sample of isotropic fused quartz. Data were taken for fundamental frequencies in the range 5-30 $\mathrm{MHz}$, all amplitudes being measured with capacitive detectors. Agreement with pulse results is surprisingly good in some cases, rather poor in others. A model is given in an attempt to explain the presence or absence of agreement between the two systems.

$$
\text { 2:35 }
$$

R5. Generation and Propagation of Finite-Amplitude Waves in Flexible Tubes. L. BJ $\phi \mathrm{RN} \phi$, Technical University of Denmark, Building 404, DK-2800 Lyngby, Denmark.-Highly reproducible finite-amplitude waves, generated by a modified electromagnetic plane-wave generator, characterized by a rise time $<0.5 \mu$ sec, peak pressures of $20-100 \mathrm{bar}$, and time constants of 5-10 $\mu \mathrm{sec}$ in the exponential pressure decay behind the front, are propagated along the axes of water-filled steel, perspex, and natural rubber tubes (30-50 $\mathrm{mm}$ i.d., 40-100 $\mathrm{mm}$ o.d.). Using new, very small piezoelectric transducers with a high natural frequency, pressure-time functions are measured at different axial and radial tube positions. The frequency domain equation for wave propagation in elastic fluid-filled tubes is developed and solved numerically on an IBM 360/75 computer for water-filled tubes of steel and perspex in the frequency range $1 \mathrm{kHz}-1 \mathrm{MHz}$, giving dispersion curves for phase and group velocities of the first six propagation modes. Using the fast Fourier transform technique on the initial finite-amplitude waves together with (1) the dispersion relationship for the Fourier components, (2) a mode selection procedure, and (3) a new expression for choosing the interval of integration, the waves are "synthesized" again at different tube positions. Surprisingly good agreement between measured and calculated pressure-time functions is found. The work throws new light on the distribution of energy in the modes of propagation and on the influence of parameters of the materials considered on finite-amplitude wave propagation.
R6. Nonlinearity Considerations in Bragg Imaging of Ultrasonic Waves. M. A. Breazeale, Franklin D. Martin, * and Laszlo AdLer, Department of Physics, The University of Tennessee, Knoxville, Tennessee 37016.-Two fundamentally different techniques exist for the study of the diffraction of light by finite amplitude ultrasonic waves: (1) Diffraction of light at normal incidence can be used to determine the ultrasonic waveform. (2) Diffraction of light incident at the Bragg angle gives detailed information of a completely different nature. Each diffraction order contains an image of the ultrasonic wavefront. Therefore, objects placed within the ultrasonic field are imaged in the diffraction orders. When a finite amplitude ultrasonic wave is used, an ultrasonic wave transmission filter allows one to distinguish the images produced by the second harmonic from those produced by the fundamental. The image produced by the second harmonic is twice as large as that produced by the fundamental and appears at the position of the fundamental's second order. In addition, at large amplitudes, multiple scattering of light by the fundamental ultrasonic wave can produce higher Bragg diffraction orders. These higher orders contain multiple images. Two images appear in the second order, three in the third, etc. [Research supported by the Office of Naval Research.]

* Present address: Lockheed Electronics Co., Inc., 16811 El Camina Rd., Houston. Tex. 77058 .

\section{3:05}

R7. Optical Determination of the Nonlinearity Parameter of Water. B. D. COOK and N. C. Martin, Cullen College of Engineering, University of Houston. Houston, Texas 77004.The optical methods of investigating ultrasonic waves played an important role in the early investigations of finite-amplitude acoustics, particularly in experimental determinations of the nonlinear parameter $B / A$ of water. However, after thermodynamic methods were discovered for obtaining $B / A$, the optical methods did not then, and have not as yet, yielded values in agreement. This paper discusses a new attempt to evaluate $B / A$ for water, utilizing the optical techniques and taking in account a correction for the nearfield of the transducer. Assumptions made for smoothness of the second harmonic nearfield and the accepted value of the piezooptic coefficient are discussed.

\title{
Session S. Speech Communication IV : Pitch and Frequency
}

\author{
Robert E. McGlone, Chairman
}

Department of Speech Communication, State University of New York at Buffalo, Buffalo, New York 14226

\section{Contributed Papers}

S1. Spectral Effects of Subglottal Cavity System. G. FANT, K. Ishizaka, J. Lindquist, and J. SundBERG, Department of
Speech Communication, Royal Instiute of Technology, S-10044, Stockholm, Sweden.- Aspirated sounds display shifts of for- 
mant frequencies and new formants are added. These can typically be observed in the aspirative part of stop consonant bursts. A quantitative modeling of this influence has been performed on the basis of subglottal impedance data and simple geometry approximations of the subglottal system. The influence of subglottal coupling on voice-source characteristics is discussed.

S2. Acoustical Features and Perceptual Cues of the Four Tones of Standard Colloquial Chinese. C-K. Chuang and SHIzuo Hıкі, ${ }^{*}$ Department of Electrical Engineering, Tohoku University, Sendai, Japan.-Monosyllabic, bisyllabic, and trisyllabic words of various phonemic constitutions were spoken with the four tones of standard colloqual Chinese by three speakers, two male and a female, born in Formosa, and the typical pitch patterns of the four tones for each speaker were extracted from these speech samples. Effects of the position of the syllable and the context of the kind of tone in a word on the average pitch frequency, range of change in pitch frequency, and duration of the typical pitch patterns were also analyzed. Then, the listening test was conducted to identify the kind of tone of the speech samples, and perceptual cues of the four tones were investigated through the confusion among tones, which was characteristic of each of native and nonnative listeners groups and each of nonsense and meaningful words groups. Based on the results of the acoustical analysis and the listening test, a set of essential features of the four tones were derived, and a generative model of pitch pattern of the tone accent was proposed. The results were examined utilizing listening test with the synthetic speech generated by the model. Effect of intensity pattern on the tone accent was also discussed.

* Also at Res. Inst. of Elec. Comm.

S3. Rule Synthesis of Simple English Sentences. J. P. OLIVE, Bell Telephone Laboratories, Incorporaled, Murray Hill, New Jersey 07974. - Simple English sentences were generated by concatenating words spoken in isolation with minimum modifications of the synthesis parameters. The sentence forms were: Article-(adjective)-subject-verb-article-(adjective)-object. The two adjectives may or may not be present. A dictionary of 266 words specified by formant data with the duration of the words adjusted by linear interpolation to a reasonable duration for connected speech was stored. Pitch rules for the different sentences were derived from an average sentence pitch contour. Words were concatenated with no further duration adjustment, and formant smoothing was applied only at voiced adjacencies for one set of data of both the preceding and the following words; the pitch, however, was replaced by the rule pitch. The rule-generated sentences were compared with sentences obtained from formant analysissynthesis of naturally spoken sentences, rather than with the naturally spoken sentences themselves, because formant analysis-synthesis introduces a characteristic nonnatural quality that is not a consequence of the rule-prosody. These comparisons demonstrated that the quality of the rule concatenated sentences is comparable to the quality of the resynthesized speech.

S4. The Perceived Pitch of 23 Prevocalic Constants. M. M. Peterson and C. W. Asp, Department of Audiology and Speech Pathology, University of Tennessee, Knoxville, Tennessee 37916.-Twenty-three prevocalic consonants were arranged on tape in 253 different pairs (e.g., /pa-sa/). Twenty-three normal-hearing listeners utilized the method of paired comparison to judge each pair according to the consonant that was "higher" in pitch. Coefficients for consistency for all listeners and the coefficient for agreement among listeners were significant. The perceived pitch among the 23 consonants was significantly different. Voiceless consonants were higher than voiced consonants. Fricatives and affricatives were higher than nasals. Place of articulation was different, but no particular order was apparent. The consonants were rank ordered from low to high pitch from the responses of all 23 listeners and also from the 10 best listeners. Both rank orders were compared to Templin's norms and Poole's norms for the acquisition of speech sounds. All correlation coefficients were positive, but only the coefficient between Poole's norms and the 10 best listeners reached significance. In addition, the two rank orders were compared to Guberina's ordering of consonants according to "optimal octaves." Both coefficients were significant.

S5. Analysis and Evaluation of Time-Domain Techniques for Pitch Ertraction. Hiroya Fujisaki and Yoshinisa Tanabe, Engineering Research Institule, Faculty of Engineering, University of Tokyo, Bunkyo-ku, Tokyo, Japan.-Recent techniques for pitch extraction are mostly based on detection of short-time periodicity of certain time functions, such as autocorrelation function or cepstrum, derived by some transformations of the original speech signal. No systematic studies, however, have been made for the analysis and quantitative evaluation of the performance of these techniques. This paper presents results of both theoretical and experimental investigations on this problem. Deterioration in the observed periodicity is caused partly by the nonstationarity of the signal itself, and partly by the finite length of the time window of observation as well as its relative phase to the excitation signal. These effects are theoretically estimated for a given set of parameters of the speech signal and the analysis procedure, and lead to quantitative evaluation and comparison of various techniques thus far proposed. Results of the theoretical analysis are then confirmed and supplemented by experimental analysis of performances of these techniques for natural speech.

S6. Some Statistical Characteristics of Voice Fundamental Frequency. YoshIYUKI HORII, Purdue University, Lafayette, Indiana 47907.-Distributional measures such as mean, median, standard deviation, $90 \%$ range, and $50 \%$ range of fundamental frequency have been of ten used to characterize voices. This study reports behavior of such statistical measures for each of six talkers as a function of sample size (from 3.6 up to $200 \mathrm{sec}$ ). Fundamental frequencies were obtained by a computer program that used a peak-picking method. The results showed that, for each script reading by a given talker, the standard deviations of the means converge fairly quicklythat is, to within about $2 \mathrm{~Hz}$ of the total mean at a sample size of about $1 \mathrm{~min}$. However, when all the various readings from even a single talker are used to get the total mean, the convergence was much slower, indicating that differences among the scripts and day-to-day variations in repeated readings are significant and must be taken into account when interpreting statistical measures of fundamental-frequency variation. Median convergence behavior was the same. Implications of these findings to studies of normal and disordered speech are discussed. [This research was supported in part by the Air Force Cambridge Research Laboratories.] 


\author{
Session T. Musical Acoustics
}

\author{
A. BENade, Chairman
}

Case Western Reserve University, Cleveland, Ohio 44106

\title{
Contributed Papers (12 minutes)
}

\section{$1: 15$}

T1. Acoustic Study of Register Equalization in Singing. JoHN W. LARGE, School of Music, Universily of Soulhern California, Los Angeles, California 90007.-A paired comparison technique in which fundamental frequency $(330 \mathrm{~Hz})$, phonemic category, /a/ and sound level (comfortable but equal) were held constant with register as the variable of primary interest was utilized with subjects who sang tone pairs varying in degree of audible-register timbre difference or equalization. The paired tones were presented to a panel of 12 expert listeners for judgments of register identification and ratings of register equalization. The vowels produced were labeled by a trained phonetician. These judgments were compared with the acoustic records from a Sonograph modified to permit 24-msec sections to be made at five positions within selected vibrato cycles: two peaks, two slopes, and a valley. The mean values for each partial and each subject were pooled in mean spectra for comparison of female chest and middle registers. The values for the paired tones judged to be "best equalized" were also compared across registers and with the spectra of the "least equalized" paired tones. It was found that chest register was characterized in general by greater energy in the partials above the third partial, especially partials 4 and 5 , and that the middle register had a stronger fundamental.

$$
1: 30
$$

T2. Observations on the Vital Capacity of Singers. JoHN W. LARGE, School of Music, University of Southern California, Los Angeles, California 90007.-The vital capacities of 20 male and 20 fernale singers attending a summer workshop program of the National Association of Teachers of Singing were measured by a Collins respirometer. The data were compared with predicted vital capacity based on Baldwin's formulas for normal subjects. A wide range of results was obtained suggesting that singing teachers should not take for granted an increase in vital capacity due to the activity of singing. On the average, however, the singers showed roughly $16 \%$ greater measured vital capacity than predicted for the corresponding age and height. The highest percentage of increase in measured vital capacity was achieved by a male subject, roughly $50 \%$. The highest value among the female subjects was $43 \%$. It was also interesting that the elder age group (above 50 years) still showed some increase in vital capacity. There appeared to be little correlation between the increase in vital capacity and either body surface or number of years of singing experience. The study furnishes an example of potentially useful research that might be conducted at NATS conventions and workshops.

\section{$1: 45$}

T3. An Electronic Violin. J. Kohut and M. V. Mathews, Bell Telephone Laboratories, Murray Hill, New Jersey 07974.The electronic violin described here was developed from data obtained in previous studies in which the resonances of a violin body were simulated electronically. The mechanical part of the electronic instrument consists of a violin without a body. Transducers, sensitive to string motions at the bridge, drive 28 electrical resonances which substitute for those of the normal violin body. The filtered, amplified voltages are applied to a loudspeaker. Violinists report the electronic instrument is easier to play than a conventional one because it responds faster and its strings are easier to bow. By changing the amplitudes of various resonances, the instrument can be quickly adjusted to produce a wide range of tone qualities. Nonlinear enhancements of responsiveness and tone quality are possible.

T4. Viola with Electronically Simulated Body Resonances. W. S. Gorrill, Physics Deparinzent, Hofstra Universily, Hempstead, New York 11550.-A viola, somewhat similar to Mathews' violin, has been built with electronic simulation of the body normal modes. Since subjective evaluation of the tone by musicians in actual performance is one of the major objectives, the appearance and apparent sound source location have been kept as close as possible to those of a normal instrument. A battery power supply is used. The effect of broadband versus narrow-band filters for the higher frequency regions has been investigated. The instrument is rather easier to play than a normal 15 -in. viola, but provides depth and power of tone more comparable to a 17 -in. instrument. The viola will be demonstrated.

\section{$2: 15$}

T5. Interaction of the Clarinet Reed with an Air Column Having a Single Resonance. JoHN Backus, Department of Physics, University of Southern California, Los Angeles, California 90007.-A study of the interaction between the reed and air column in the clarinet is underway, using a synthetic clarinet reed in a mouthpiece adapted to accomodate resonant air columns of various kinds. Measurements of the air flow into the reed-mouthpiece combination mounted in an artificial embouchure show the negative resistance characteristic necessary to sustain oscillations in an attached resonant air column; the value of this resistance is varied by changing the gap between the reed tip and the mouthpiece. A system resembling a stretched-out Helmholtz resonator and having a single pronounced resonance was made to oscillate by attaching it to the mouthpiece and blowing on the artificial embouchure. The input impedance of the system at its resonance frequency was measured on an impedance tube, and it was expected that the system would oscillate when this input impedance was approximately equal to the negative resistance of the reed-mouthpiece combination. However, experiments showed that oscillations did not take place until this negative resistance was about half the input impedance. [This work was supported by a grant from the National Science Foundation.] 


$$
\text { 2:30 }
$$

T6. Spectrum Development with Playing Level in Wind Instruments. W. E. Worman, Case Western Reserve University, Cleveland, Ohio 44106.- -Starting from the theory of musical wind instruments proposed by Benade and Gans and further developed by the author, it is possible to draw very general conclusions about the development of the spectrum in the mouthpiece as a function of loudness. A threshold blowing pressure exists below which no oscillations are possible. Near threshold, the pressure amplitude of the fundamental is proportional to the square root of the excess of the blowing pressure over its threshold value. The pressure amplitudes of the higher harmonics can be written as $P_{n}=\left(P_{1} / E_{n}\right){ }^{n}\left|Z_{n}\right|$, where $P_{n}$ is the pressure amplitude of the $n$th harmonic, $\left|Z_{n}\right|$ is the magnitude of the input impedance of the bore at the frequency of the $n$th harmonic, and $E_{n}$ is a constant that can be evaluated experimentally and calculated for simple systems. Experiments by Benade on many types of wind instruments (including clarinet, bassoon, trumpet, and saxophone) show that this form of spectrum development takes place over a surprisingly large range. In many instruments, the range is about $40 \mathrm{~dB}$ in the pressure amplitude of the fundamental (pianissimo to mezzoforte). Observed departures from this behavior may be understood in terms of the same basic theory.
$2: 45$

T7. Real-Time Music Analysis for the Performer. INGo R. Titze and Wrlliam J. Strong, Department of Physics, Brigham Young University, Provo, Utah 84601.-Waveform, pitch, dynamics, and 32 harmonic components of a tone with a specified nominal pitch are displayed on a computer-driven CRT. The rate of display is approximately $10 / \mathrm{sec}$, thus presenting essentially real-time analysis. The nominal pitch and the expected pitch deviation are specified by the performer. The pitch deviation can range from a quarter step to a whole step above and below the nominal pitch. The performer can visually adjust his average pitch, monitor his vibrato rate and range, check his dynamics, and observe his spectrum while he sings or plays into the microphone. As an additional feature, the spectrum may be averaged over any specified time interval. The spectrum is obtained by taking a 64 point FFT over a single period of the waveform. The period is determined by sweeping the pitch over the specified range and selecting the best value on the basis of the minimum of a one-cycle average of the waveform and a "fractional period correlation" of adjacent cycles. The present system is comprised of a $30-\mathrm{kHz}$ A/D converter and a PDP-15 computer system with associated graphics.

Wednesday, 19 APRIL 1972

EMPIRE State Room, $1: 30$ P.M.

\title{
Session U. Noise I : Propagation of Sound in Ducts
}

\author{
IsTVÁN VÉR, Chairman \\ Bolt Beranek and Newman Inc., Cambridge, Massachusetts 02138
}

Contributed Papers (12 minutes)

$1: 30$

U1. Effects of High-Intensity Sound on Muffler Element Performance. M. P. SACKS AND D. L. AlLEN, Department of Mechanical Engineering, University of Toronto, Toronto, Onlario.-This paper presents the results of an experimental investigation designed to study the effect of high-intensity sound on muffler element performance. Five prototype mufflers were built and tested over a wide range of frequency and intensity. The mufflers were chosen as being representative of basic single element and combination reactive mufflers. The test facility consisted of an acoustic transmission line driven by an electromagnetic sound source and terminated anechoically. Attenuation data is presented superimposed on curves derived from linear acoustic theory. Conclusions are stated for expansion chamber mufflers at higher SPLs than could be produced by the test equipment by using constriction-tube data and acoustic similarity between these elements. It is shown that the expansion chamber muffler will operate as a linear element when subject to incident SPLs of 165-180 dB depending on muffler attenuation. Mufflers containing resonator elements show no deviation from a linear theory when subject to SPLs of the order of $160 \mathrm{~dB}$ except at points near a resonant frequency.

\section{$1: 45$}

U2. Acoustic Impedance of Perforated Plate Liners with TwoFrequency Excitation. A. N. ABDELHAMID, Faculty of Engineer- ing, Carleton University, Ottawa, Ontario, K1S 5B6, Canada.The acoustic impedance of a lining material has been previously shown to be dependent on the noise spectrum impressed upon the liner and several analytical models for its determination have been recently proposed. The results of these models, however, seem to be in very good agreement with experimental data for single-frequency excitation, but not as good with the limited available data for two-frequency excitation. In this paper, an impedance tube with two sound drivers at one end is used to investigate the performance of perforated plate liners under two-frequency excitation. The two frequencies varied from 800 to $2000 \mathrm{~Hz}$ with SPLs at the liner up to $145 \mathrm{~dB}$. The plate thickness varied from 0.05 to $0.20 \mathrm{in}$. with open area ratio ranging from 0.05 to 0.10 . The experimental results indicate that, for the same SPL at the liner, the effect of excitation at the liner resonant frequency on the acoustic resistance at other frequencies is considerably higher than the reverse effect. Based on the experimental data obtained, modifications to the previously proposed impedance models are discussed.

\section{2:00}

U3. Sound Attenuation in Ducts Lined with Nonisotropic Material. U. J. Kurze AND I. L. Vér, Bolt Beranek and Newman Inc., Cambridge, Massachusetts 02138.-This paper deals with the sound attenuation in rectangular ducts with nonisotropic lining. The nonisotropy may be due to the basic structure of the fibrous lining material or it may be deliber- 
ately created by rigid partitioning of the lining perpendicular to the duct axis. The propagation constant in the axial direction is obtained in the form of a transcendental equation which includes as special cases the locally reacting lining investigated by Morse and Cremer for infinite flow resistance in the direction of the duct axis, and the homogeneous lining as treated by Scott. A simple approximate solution of the transcendental equation for low frequencies indicates that optimum attenuation of the fundamental mode is achieved using a nonisotropic lining with a flow resistance in the direction of the duct axis that increases with increasing frequency. At middle and high frequencies, the propagation constant is evaluated numerically using a Newton-Raphson scheme for several typical silencer geometries.

$$
2: 15
$$

U4. Acoustic Propagation in a Waveguide with a Transverse Concentration Gradient. RichaRd F. SALANT, Massachusetts Institute of Technology, Cambridge, Massachusetts 02139.Acoustic propagation in a two-dimensional waveguide containing a two-component gaseous mixture with transverse concentration gradient, has been investigated analytically. It has been shown that if the steady density is uniform, the sole effect of a concentration gradient is to produce a variable sound speed. However, if the steady temperature is uniform, the medium must be treated as a two-component mixture. For the latter case, the eigenvalues and eigenfunctions have been computed.

$$
2: 30
$$

U5. Development of a Computer Program for the Determination of Sound Absorption in Cylindrical Ducts Lined with Porous Materials. A. G. JhaverI, Harris F. Freedman, and Associates, Mercer Island, Washington 98040.-A computer program has been developed that allows a determination to be made of the sound absorption in cylindrical ducts. The computer program is based on a realistic mathematical mode for computing sound absorption of a very long cylindrical duct lined with porous material of uniform thickness. For simplicity, it is assumed that there is no airflow in the duct. The program computes sound absorption of the fundamental mode, the solution being for a single-frequency component of an unaveraged time signal. These calculations must be repeated for each of the frequencies being considered, a special feature of the program. The results of this program are in reasonably good agreement with the experimental data. The predicted curve has both qualitative and quantitative agreement with that obtained from actual test results, in terms of the amplitude and location of the resonance.

Wednesday, 19 April 1972

Maple Leaf Room, $2: 00$ P.M.

\title{
Session V. Machine Noise III : Determination of Sound Power
}

David Lubman, Chairman

\author{
D. Lubman \& Associates, 23727 Crosson Drive, Woodland Hills, California 91364
}

This session is a continuation of the two special sessions on Noise Measurement.

\section{Contributed Papers}

\begin{abstract}
V1. Experimental Investigation of the Statistics of SoundPower Radiation in a Reverberant Room. Wayne F. SMITH and J. Ronald BaIley, Center for Acoustical Studies, Department of Mechanical and Aerospace Engineering, North Carolina State University, Rayleigh, North Carolina 27607.- The measurement of sound-power injection into a reverberant room is subject to statistical uncertainties owing to the large fluctuations of SPL with variation of source and/or receiver position. Low-frequency pure-tone sources generally require many microphone and source positions if radiated sound power is to be accurately measured. An experimental investigation of lowfrequency pure-tone sound radiation into a reverberant room has been conducted using both an 8-in. loudspeaker and a 30in. loudspeaker at five source positions. The resulting standard deviation of sound-power output of the smaller speaker with source position is in excellent agreement with existing theory if the data is adjusted to account for the finite number of independent microphone readings. Standard deviation of the sound-power output of the larger speaker with source position is significantly lower than the theory at frequencies below $500 \mathrm{~Hz}$. [NASA is acknowledged for partial sponsorship of this work.]
\end{abstract}

V2. Practical Sound-Power-Level Measurements. L. J. Eriksson, Sonotek, New Berlin, Wisconsin 53151.-The measurement of sound-power level in semireverberant rooms is discussed. The standard equations for calculating sound- power level in reverberant and semireverberant rooms are compared. It is shown that for many practical measurements, the two equations produce essentially the same result. In particular, when the area of the room is equal to four times the area of the hemisphere surrounding the source, the results are identical under normal room conditions. This is also true for a cube-shaped room, if the distance from the source is onehalf the wall dimension. Sound-power-level measurements made in three semireverberant rooms on a small electric motor are reported to illustrate this result. The effect of a partial enclosure on the sound-power level is also discussed.

V3. The Effect of the Rotating Vane on the Averaged Sound Pressure in Reverberation Chamber. JIRI TICHY, Department of Architectural Engineering, The Pennsylvania State University, 101 Engineering " $A$ " Building, University Park, Pennsylvania 16802, AND DOUGLAS ANDERSEN, Engineering Acoustics Program, The Pennsylvania State University, 203 Engineering " $D$ " Building, University Park, Pennsylvania 16802. - The modulation effect of the rotating vane in the reverberation chamber and the consequences on the soundpower measurement at single frequencies [J. Tichy, "The Effect of Boundary Conditions on the Statistical Properties of the Sound Field in Enclosures," J. Acoust. Soc. Amer. 50, 98(A) (1971)] has been further studied. The vane causes the amplitude and frequency modulation of the sound field, which results in line frequency spectrum of the sound in the 
chamber. Therefore, several modes or modal groups can be excited. The time average value of the sound pressure at a certain point depends on the vane speed. The statistical properties of the sound-pressure amplitude have been studied as a function of microphone location and damping of the chamber for various vane speeds.

WEDNESDAY, 19 APRIL 1972

BALLROOM, 3:30 P.M.

\author{
Session W. Plenary Session. Three Perspectives on Acoustics \\ John C. Johnson, Chairman \\ Pennsylvania State University, State College, Pennsylvania 16801 \\ Invited Papers (40 minutes)
}

\begin{abstract}
3:30
W1. Acoustics as a Physical Science. Rogert T. Beyer, Department of Physics, Brown Universily, Providence, Rhode Island 02912.-A comprehensive review of the field of acoustics, from the perspective of its relationship to physics, has been completed. Contrary to conclusions of many colleagues in the field of physics, acoustics retains a vital role in the search for knowledge about our physical universe. Additionally, the physical concepts developed in studies of acoustics continue to play an important part in support of the several aspects of acoustics that are categorized as parts of other fields. These viewpoints are supported by several selected examples in order to provide a meaningful understanding by workers in the several subfields of acoustics.
\end{abstract}

W2. Acoustics as a Human Science. S. S. Stevens, Laboratory of Psychophysics, Harvard University, Cambridge, Massachusetts 02138. - There was a time when acoustics was only a human science, but this changed as philosophers learned to use mathematical and other measurement tools for ordering and validating their thought processes. This century has seen many changes in the description of the field as a whole, and the human aspects continue to be among the most perplexing. The highlights of accomplishments to date, some observations on our current status of knowledge, and some projections into the future are presented for your entertainment-if not for your information.

$$
\text { 4:50 }
$$

W3. Applied Acoustics and Its Role in Modern Society. Daniel R. FlynN, Applied Acoustics and Illumination Section, National Bureau of Standards, Washington, D. C. 20234.-Acoustics has been a wonderful science for the thousands of workers in that field. Yet, we find ourselves today with an unfulfilled wanting for rewards from the scientific accomplishments. It is easy to name dozens of specific problems of very practical importance today which need solutions based upon these accomplishments. These problems cover a wide range-from health, pleasure, and other human problems to engineering problems of all sorts, including excavation, chemical reactions, sonar, and so forth. Some of these problems and proposed solutions will be described in terms of real world needs of today.

WEDNESDAY, 19 APRIL 1972

SOCIAL Hour $6: 30$ P.M.

ReNDEZVOUS ROOM

BANQueT $7: 30$ P.M.

TERrace Room

Presentation of the Biennial Award to Robert D. Finch

Encomium by Douglas Master

Speaker: Dr. F. KarL Willenbrock, Director, Institute of Applied Technology. National Bureau of Standards-"Noisy Systems-Physical and Managerial" 
$83 R D$ MEETING A ACOUSTICAL SOCIETY OF AMERICA

ThURSDAY, 20 ApriL 1972

Buffalo Room, 9:00 A.m.

\title{
Session X. Physical Acoustics I : Cavitation
}

\author{
Hugh G. Flynn, Chairman \\ University of Rochester, Rochester, New York 14627
}

Invited Papers (30 minutes)

9:00

X1. Bubble Dynamics. Milton S. Plesset, California Institute of Technology, Pasadena, California 91109.--Recent work on vapor cavities and gas-filled bubbles will be discussed.

$$
9: 30
$$

X2. Dynamics and Oscillation of Nonspherical Bubbles. D. Y. HsıE, Divison of Applied Mathemalics, Brown University, Providence, Rhode Island 02912.-A brief survey is first given on the linear stability analyses of the notion of spherical bubbles. These include the stability of the growth and collapse of bubbles in an incompressible liquid and the stability of bubbles in oscillation. The linear stability formulations are extended to include the effect of the compressibility of the liquid and the effect of heat and inass transfer across the bubble wall. Then a variational method is formulated to deal with the case when the nonsphericity of the bubble is not small. This method leads to some new approaches for approximate treatments of dynamics and oscillations of nonspherical bubbles. Some examples are treated and numerical results obtained.

\section{0:00}

X3. Laser-Induced Cavitation. W. Lauterborn, Driltes Physikalisches Institut der Universität Göttingen, N-34 Göttingen, Germany.-When intense laser light is focused into liquids, bubbles can be produced in the vicinity of the focal point. The dynamics of these bubbles is investigated by highspeed photography with picture repetition rates up to $10^{6} \mathrm{frames} / \mathrm{sec}$. Single spherical bubbles can be produced. In water, they can be said to be the experimental realization of the Rayleigh bubble model. Results on both spherical and nonspherical bubble collapse, rebound, and subsequent collapses and rebounds will be reported showing the sequence of events leading to the cloud of minute bubbles of ten observed after the collapse of a big bubble. Repeated jet formation of the same bubble seems to be the major sticide mechanism. Shedding of minute bubbles occurs by gas entrainment into the jet. Spherical undisturbed collapsing bubbles remain spherical down to very small radii being heavily distorted a few microseconds later during rebound. Nonlinear spherical bubble oscillations are investigated in silicone oils. The stability of the spherical shape seems to be enhanced by viscosity. A comparison is made with calculated nonlinear bubble oscillations damped by viscosity.

\section{$10: 30$}

X4. Vortices and Turbulence as a Cavitation Nucleation Mechanism. R. D. FINCH, Department of Mechanical Engineering, University of Houston, Houston, Texas 77004.--Several experiments using liquid helium as a medium of study have indicated that superfluid vortices or turbulence enhance ultrasonic cavitation. The possible mechanisms of this process and its possible applicability for normal liquids are discussed.

11:00

X5. Investigations of Acoustic Cavitation. L. M. LamChev and V. A. Akulichev, Institute of Acouslics, Moscow, USSR.

\section{Contributed Papers (12 minutes)}

\section{$11: 30$}

X6. Differential Equation Approximation to the Expansion of an Underwater Cylindrical Gas Bubble. David Epstern, State University of New York, Maritime College, Fort Schuyler, Bronx, New York 10465.- The underwater cylindrical gasbubble-expansion problem has been discussed in earlier reports [D. Epstein and J. B. Keller, J. Acoust. Soc. Amer. 44, 395(A) (1968); J. Acoust. Soc. Amer. 46, 117 (A) (1969)]. It was solved in the acoustic approximation, by treating the water as slightly compressible, and a system of nonlinear integrodifferential equations for the bubble radius was derived. In recent studies, a differential equation (DE) approximation was applied to the problem [R. A. Wentzell et al.. J. Acoust. Soc. Amer. 48, 1283-1286 (1970); A. I. Joffe et. al., Sov. Phys.-Acoust. 13, 180-183 (1967)]. Here it is shown that by making certain approximations, a single $\mathrm{DE}$, similar to those used in the papers cited above, may indeed be formally derived from the set of integrodifferential equations. The DE has a logarithmic term that causes the bubble pulsations to increase eventually without bound. However, in the early stages of the expansion, the DE solution compares favorably with the rigorous one. Thus, for certain purposes, it may be expected to yield useful results.

$$
11: 45
$$

X7. Effect of Surface Tension on the Cavitation Threshold of Water. L. A. Crum, J. B. Gallemore, and D. A. Nordling, Michelson Physical Laboratory, U.S. Naval Academy, Annapolis, Maryland 21402. - The threshold for transient cavitation in degassed water has been measured in a stationary wave 
system as a function of surface tension. A surfactant was used to vary the surface tension of water from approximately 70 to $30 \mathrm{dyn} / \mathrm{cm}^{2}$. The cavitation threshold was found to vary inversely with the surface tension in disagreement with the theoretical predictions of Apfel [J. Acoust. Soc. Amer. 48, 1179-1186 (1970)]. Glass capillary tubes were used to construct macroscopic models of microscopic crevices thought to nucleate cavitation. The crevices were coated with polystyrene in order to obtain a nonwettable surface. Measurements made of the receding contact angle of an air-water interface moving in the model crevice showed a critical dependence of the contact angle upon surface tension. Motion pictures will be shown of the receding contact angle as a function of surface tension and how this receding interface may nucleate cavitation. If an empirical relationship is made between the contact angle and the surface tension, and this empirical dependence used in Apfel's theory, then good agreement is obtained between theory and experiment. [Work supported by the Office of Naval Research and the Naval Academy Research Council.]

\section{2:00}

X8. Thresholds for Surface Waves and Subharmonics Associated with a Single Bubble. D. L. STORM, Physics Department, University of Vermont, Burlington, Vermont 05401.-When a gas bubble is subjected to an acoustic field, waves are seen on its surface after the pressure amplitude $P$ of the ambient field increases beyond some threshold $W$. Similarly, a threshold pressure amplitude $S$ has been observed for excitation of a subharmonic response of order one-half. For the work reported here, the thresholds $I^{\prime}$ and $S$ were measured in the frequency range 0.1 to $3 \mathrm{kHz}$. In a typical experiment, a rather large single bubble, with frequency $f_{0}$ of volume resonance in the range $0.5-1.5 \mathrm{kHz}$, was subject to a plane standing wave in a liquid, while prevented from rising by a thin plastic film. Studies were made over a range of frequencies $f$ extending above and below $f_{0}$. The behavior of the air-liquid interface was observed, and a hydrophone in the liquid near the bubble monitored the pressure at $f / 2$. The output of another hydrophone placed some distance away from the bubble indicated $P$. Both thresholds $I Y$ and $S$ were found to exhibit minima for $f=f_{0}$. For a bubble in water resonant near $1 \mathrm{kHz}$, the lowest value for $\mathrm{l}$ was several millibars, and that for $S$ was somewhat greater.

\section{$12: 15$}

X9. Light Scattering by Ultrasonic Cavitation in Liquid Helium II. D. B. Greene AND P. L. Marston, Stanford University, Stanford, California 94305.-Microscopic bubbles have been generated in liquid helium II with $50-\mathrm{kHz}$ ultrasonic sound. We have observed that light is scattered from these bubbles and is intensity modulated at the fundamental, harmonics, and subharmonies of the driving frequency. The SPL for the onset of scattering coincides with the second audible threshold described by Jarman and Taylor [P. D. Jarman and K. T. Taylor, J. Acoust. Soc. Amer. 39, 584 (1956)]. Apparatus is now being constructed to photograph bubbles and to refine our original scattering measurements in order to measure their size distribution. We also hope to study the interaction of bubbles with quantized vorticies.

* Work supported in part by The Nat. Sci. Found.

ThURSDAy, 20 ApriL 1972

Empire State Room, 9:00 a.m.

Session Y. Shock and Vibration III : Analytical Techniques

Dennis P. Malone, Chairman

State University of New York at Buffalo, Buffalo, New York 14226

\section{Contributed Papers (12 minutes)}

\section{$9: 00$}

Y1. Studies of Periodic and Transient Deformations by the Method of Double-Pulsed Holographic Interferometry. PETER Shajenko, New London Laboratory, Naval Underwater Systems Center, New London, Connecticut 06320.- Studies of deformation of mechanical structures subjected to periodic and transient excitations have been made by the method of double-pulsed holographic interferometry. A $Q$-switched ruby laser with pulse duration of $20 \mathrm{nsec}$ and coherence length in excess of $1 \mathrm{~m}$ was employed. To demonstrate vibration analysis, a thin steel plate was driven over a range of frequencies and amplitudes. The amplitude in order of $0.1 \mathrm{~mm}$ was recorded; 288 fringes in a thin plate are clearly readable. Shock waves were recorded in a thick plate, transducer housing, and a solid rod. In a thick plate, an amplitude of 220 fringes was obtained. In loud-speaker studies, the various effects such as paper stiffness of the cone, cone asymmetry, etc., on the frequency response can be deduced from the extremely fine details in the mode structure. The loudspeaker was driven to provide the power output level up to $95 \mathrm{~dB}$ at a number of frequencies and the details of characteristic mode structure are presented. The technique described is characterized by the high dynamic range and the extremely high fringe contrast attainable; the technique is relatively insensitive to spurious vibrations. As a result it is applicable to testing under diverse operational conditions.

\section{$9: 15$}

Y2. Plane-Wave Admittance of an Elastic Half Space. ANANT K. NigaM, Bell Telephone Laboratories, Murray Hill, New Jersey 07974. - The ratio of complex amplitudes of the normal velocity and the normal stress (i.e., the admittance) of a plane displacement wave traveling on the surface of an elastic half space is studied. The admittance is computed as a function of the traveling wave parameters of the surface wave. Four ranges of phase velocity of the surface traveling wave have been identified, which are the ranges of velocity separated by the three characteristic speeds in the half space (the Rayleigh, shear, and dilatational speeds). In each of the four velocity ranges, the plane-wave admittance of the elastic half-space exhibits a different functional form. Simple interpretations for each functional form of the admittance are provided in analogy with a single-degree-of-freedom, mass-spring-dashpot system. The correlation of half-space behavior (to traveling waves on the surface) with the nature of the vibration pattern within the half-space is discussed. 
Y3. Response of a System Consisting of $N$ Coupled Elements. A. J. Tucker and G. Maidanik, Naval Ship Research and Development Center, Bethesda, Maryland 20034.--The impulse response function method, which was developed previously by Tucker and Maidanik [J. Acoust. Soc. Amer. 51, 87(A) (1972)] is extended to a system containing $N$-coupled elements where the coupling acts only at a point of each element. The essence of the method is that it considers the coupling to be a force field acting on an uncoupled element of the system. Then this coupling force field together with the external driving field and the impulse response functions of the uncoupled elements determines the response of the coupled system to an arbitrary external driving field. This method does not require a priori definition of the exact nature of the individual elements; it requires only the existence of the elemental impulse response function and knowledge of the type of coupling. As a specific example, the general result is utilized to investigate a system where the elements are identical, the coupling impedances are identical, the points of attachment on each element are similar, and a point driving force is applied to only one element. Then the effect on the response of the system of various degrees of coupling and the number of elements in the system is examined.

Y4. Experimental Study of Transient Response Spectra of the Double Acoustical Resonator. N. N. REDDY, Lockheed Georgia Co., Marietta, Georgia 30060, AND R. L. LowERY, School of Mechanical Engineering, Oklahoma State University, Stillwaler, Oklahoma 74074.-Experiments were conducted to study the pressure transient response of an adjustable double acoustic resonator. The excitation was provided by a transducer at the end of a 32-ft plane-wave tube. Pressure histories were measured in both cavities of the resonator. Pressure magnifications as high as 6.0 were measured in one cavity. The experimental results are compared with Helmholtz theory.

10:00

Y5. Capacitance Microphone Dynamic Membrane Deflections. J. E. WARREN AND A. M. BrzezINSKI, Bell Telephone Laboratories, Indianapolis, Indiana, AND J. F. HAMlLTON, Department of Mechanical Engineering, Purdue University, Lafayette, Indiana 47907.-In order to describe the operation of capacitance microphones accurately, it is necessary to have solutions for both the static and dynamic deflections of the moving electrode. The authors have previously presented numerical solution approaches to the static deflections caused by the electrical biasing of the microphone. The present paper describes an application of finite difference methods to the differential equations describing the dynamic membrane motion due to the acoustic excitation. The solutions include the effects of the static deflections and the motion of the thin air film between the microphone's electrodes. The numerical results are compared with published results for simplified stationary electrode geometries and with experimental data on more complicated geometries at different tension levels. The predicted mode shapes and resonant frequencies change greatly depending upon the magnitudes of the membrane tensions and the back chamber volume.

\section{0:15}

Y6. Optimal Performance of Physical Systems Subject to Impact. W. D. Pilkey and B. P. Wang, Departmenl of Aerospace Engineering and Engineering Physics, University of Virginia, Charlottesville, Virginia 22901.-A computational capability for the evaluation of the limiting performance of transient dynamic systems is described. This capability permits the peak response (acceleration, force, displacement, or stress) of a system to be computed if portions of the system are optimally controlled or isolated. Thus, the worst occurrence to a human or vehicle can be determined under prescribed impact conditions if restraints such as seat belts or bumpers were to react in a time-optimal fashion and if response constraints on the system were not violated. No design configurations of the controllers or isolators are specified. Several specific applications of the capability to human and transportation systems are presented. In terms of design, this capability allows the designer to ascertain on the basis of response specifications alone the feasibility of a proposed design; in addition, he can measure and monitor his success during the design process. This is possible because the capability provides characteristics of the theoretically best, i.e., the limiting, design concept. Without the characteristics of the limiting design, the evaluation of proposed designs can be made only by performing a multitude of analyses for each candidate design. The problem is formulated in terms of linear programming. The controllers can be nonlinear, linear, active, or passive, while the remainder of the system must be linear. The linear programming formulation permits the use of offthe-shelf computer programs for the solution and, because of the capability of standard linear programs, means that multidegree-o[-freedom, multi-isolator problems subjected to multiple, alternative sets of transient loading can be treated.

\section{$10: 30$}

Y7. Response of an Infinite Strip of Structure to Fluctuating Pressure Fields. Charles 'T. Morrow, Advanced Technology Center, Inc., Dallas, Texas 75222.-The best-known method of predicting the response of aerospace structure to intense noise fields is statistical energy analysis (SEA). This is an approximate analysis of a complicated structure. It is proposed to supplement this by a simplified model analysis-an exact analysis of a simple model to be used as a basis for inferences about real structure. The simplest model is a strip of infinite length with one-dimensional propagation characteristics for transverse vibration defined by a velocity and an attenuation constant which may depend on frequency. Responses are derived for several flight pressure fields and for a reverberant field.

Thursdax, 20 April 1972

Georgian Room, 9:00 A.M.

\author{
Session Z. Physics of Sound in the Sea II : Radiation and Scattering \\ Garland Barnard, Chairman \\ A pplied Research Laboratories, University of Texas, A ustin, Texas 78712
}

\title{
Contributed Papers (12 minutes)
}

9:00

Z1. Eigenvalues for Wave Motion in Irregularly Shaped Regions. Richard Paul Shaw and George Tai, F.E.A.S.,
State University of New York at Buffalo, Buffalo, New York 14214. - Eigenvalues are obtained by means of a Helmholtz integral equation formulation for wave resonances in two- 
and three-dimensional enclosed regions. Examples of circular and spherical regions are given to compare this approach to the simpler but less general separation of variables method. An example of a triangularly shaped two-dimensional region is given to illustrate the advantages of this approach, i.e., that only surface values of the dependent variables appear, allowing a fine grid to be used with relatively few points, as compared to a standard finite difference approach for a nonseparable geometry.

9:30

22. Experimental Observation of Monostatic Reflection by Elastic Spheres in Water. Richard H. Vogt, Louis R. DraGonette, and Werner G. Neubauer, Naval Research Laboralory, Washington, D. C. 20390.-Careful comparison of steady-state theory and experiment has shown that outstanding features such as nulls in the reflection solution vs $k a$ for spheres can be identified with precision by pulse experiments. The systematic influence of experimental parameters such as elastic constants and ambient temperature is discussed. A persistent discrepancy of $0.05 \%$ in wavenumber between theory and experiment has been observed.

\section{$9: 15$}

Z3. A Kirchhoff Solution to the Diffraction by an Elastic Wedge. T. J. EISLER AND R. NEW, The Catholic University of America, Washinglon, D. C. 20017.-The scattering of an incident plane wave from an elastic wedge has been calculated using the Kirchhoff approximation. In this method reflected and transmitted waves are first calculated using Snell's law. The boundary values obtained in this way for the pressure and its normal derivative on the surface of the wedge are then substituted into the Helmholtz integral. The radiation pattern of the diffracted wave has been calculated for infinite steel wedges in water and compared with rigid wedge solutions. Close agreement is found except in certain directions corresponding to transmitted plane waves not present in the rigid case. The effect of penetration on the diffracted wave becomes more important as the number of internal reflections within the wedge increases. The method also introduces some spurious infinites in a finite number of directions.

\section{$9: 45$}

Z4. Acoustic Backscattering from Spherical and Cylindrical Shells. Donald Brill, Naval Academy, Annapolis, Maryland 21402, H. ÜBERALL, Calholic Universily, W'ashinglon, D. C. 20017, and Peter Uginčus, Naval Weapons Laboralory, Dahlgren, Virginia 22448. - The target strength of thin spherical and cylindrical elastic shells, either air- or waterfilled, has been calculated in order to assess the effects of penetrability of the shell material on the scattering process. Calculations have been performed using both the Kirchhoff method and the Watson-Sommerfeld transformation of the Rayleigh normal-mode series. The contribution of specular reflection from the vertex can be separated out in either method, and distinguished from shadow-boundary reflections or creeping-wave contributions. Similarly as in the normalmode calculations of Diercks and Hickling [J. Acoust. Soc. Amer. 41, 380 (1967)], the penetrability effects were found to be considerable.

10:00

Z5. Experimental Measurements of the Scattering of Acoustic Waves by Rough Surfaces. P. J. Welton, H. G. FREY, AND P. G. Moore, Applied Research Laboralories, The University of Texas at Austin, A ustin, Texas 78712.-Measured scattering coefficients are presented for four randomly rough, pressurerelease surfaces that are statistically related. The scattering data were collected at frequencies of 100,200 , and $500 \mathrm{kHz}$ for forward, specular, and backscattering geometries. The ratios of acoustic wavelength to surface rms height $(\lambda / h)$ range from 6.5 to 0.33 . The wide range of scattering geometries, frequencies, and roughness included in these measurements make these data the most complete collection of acoustical scattering measurements yet available for statistically known rough surfaces.

\section{$10: 15$}

Z6. Observations of Acoustic Radiation Resulting from Incidence at the Critical Angles and the Rayleigh Angle for Plane Surfaces. Werner G. Neubauer, Naval Research Laboratory, Washington, D. C. 20390.-The resulting radiated field is observed by schlieren methods for an ultrasonic beam generated in water incident on a solid interface. Radiation outside the specular region has been observed at the longitudinal and shear critical angles and at the Rayleigh angle. A periodic spatial variation in acoustic amplitude occurs in this radiated field at the Rayleigh angle. This effect will be discussed.

\section{$10: 30$}

Z7. An Exact Solution for the Impulse Response of a Baffled Rectangular Piston. J. C. Lockwoon AND H. O. BerkTay, Applied Research Laboralories, The University of Texas at Austin, Austin, Texas 78712.-An exact solution for the pressure at any point in the field of an impulsively accelerated rectangular piston is obtained. This is a closed-form timedomain solution obtained by a geometrical approach similar to that used by Farn and Huang [J. Acoust. Soc. Amer. 43, 252-257 (1968)] for the case of a circular piston. It is expected that the impulse response obtained in the present case will be useful in calculating the nearfield for arbitrary motion of a rectangular piston. [This work was sponsored by the Office of Naval Research.]

\section{$10: 45$}

Z8. Measurement of Axial Velocity Profiles and Resulting Acoustical Fields of a Rod. L. J. Nowotny AND E. L. Hixson, Department of Electrical Engineering, University of Texas, Austin, Texas 78712 . - Sinusoidal stress waves over the frequency range $10.0-85.8 \mathrm{kHz}$ were transmitted down a cylindrical aluminum rod $12 \mathrm{ft}$ long with a radius of $0.75 \mathrm{in}$. Using a modified Twyman-Green interferometer and a high-sensitivity fast rise time, photodiode radial distributions of peakto-peak axial displacement were measured at the radiating face of the rod while it was under $\mathrm{CW}$ excitation. Measurements verified the almost pistonlike displacement profiles for low frequencies and indicated increased distortion of the face with increasing frequency. Nodal cylinders of axial displacement were found to occur beginning at about $29 \mathrm{kHz}$ with only the lowest symmetric mode propagating in the rod. Directivity patterns of the radiating rod were measured over the specified frequency range in a laboratory pool. Only the radiating rod face was exposed to water at the plane surface of a 5-ft-square aluminum baffle. Measurements taken with pulsed sinuosidal excitation applied were compared with calculations for an ideal piston in an infinite baffle.

\section{1:00}

Z9. Multipole Expansion of Sound Radiation from Moving Rigid Bodies. Thomas EIsler, The Catholic University of America, Washinglon, D. C. 20017.-The solution of the wave equation with a source term extended in space and moving as a rigid body with translation and rotation is expanded about an interior point in terms of point multipoles, which are expressed as spatial derivatives of the Lienard-Wiechert solution 
for a monopole. An application to sound radiation from moving rigid bodies is given using the theory of Ffowcs-Williams and Hawkings [Phil. Trans. Roy. Soc. London A264, 321 (1969)]. As examples, the radiation fields of a rotating ellipsoid and a sphere in accelerated rectilinear motion are estimated.

\section{1:15}

Z10. Mutual Radiation Impedance between a Circular Piston and a Piston of Arbitrary Shape. P. R. Stepanishen, General Dynamics/Electric Boal Division, Groton, Conneclicut 06340.A time-domain approach is presented to compute the mutual radiation impedance between a circular piston and a piston of arbitrary shape where both pistons are mounted in a rigid planar baffle. The approach is based on numerically evaluating the Fourier transform of an impulse response, which is equivalent to the time-dependent force on the piston of arbitrary shape resulting from the impulsive velocity of the circular piston. An integral expression over a single variable is presented for the impulse response between the circular piston and an arbitrary shaped piston. The mutual impedance between a circular piston and a concentric annular ring is investigated as a special case. A closed-form expression is derived for the impulse response, which is shown to consist of two pulses that are associated with the edges of the ring. Numerical results for the mutual impedance are then presented as a function of frequency and the impedance is shown to be zero for certain critical frequencies. The configuration of two unequal circular pistons is also discussed as a special case, and numerical results are presented for the impulse response and impedance as a function of frequency.

$$
11: 30
$$

Z11. Radiation from an Array of Transducers on a Finite Cylinder. D. T. PorTer, New London Laboratory, Naval Underwater Systems Center, New London, Connecticut 06320, AND P. H. Rogers, Naval Research Laboratory, Washington, D. C. 20390 . - It is difficult to obtain valid solutions for a large finite cylindrical array using the Helmholtz integral or sourcedensity formulations because of the density of the eigenfrequencies of the interior of the body. Near these eigenfrequencies, it is difficult to obtain valid solutions. If a hole is drilled through the cylinder, the interior eigenfrequencies of the resulting finite annular cylinder can easily be found. If the hole is large enough so that the resulting wall thickness is less than a half wavelength, then there will be no eigenfrequencies below the sonar frequency and the eigenfrequency problem is avoided. An example is given showing that this hole has negligible effects on the radiated pattern. Beam patterns for an array of six bands on a finite annular cylinder three wavelengths high, 10 wavelengths in diameter, and $\frac{1}{4}$ wavelength in wall thickness are compared with patterns for the same array on an infinite cylinder, for various depression angles. The finite and infinite cylinder patterns looked much alike, with diffraction oscillations added. The diffraction oscillations were seen on the main lobe only for depression angles deeper $\operatorname{than} 30^{\circ}$.

ThursDay, 20 APRIL 1972

MAPLE Leaf Room, 9:00 A.M.

\title{
Session AA. Noise II : Noise Control for Vehicles and Stationary Noise Sources
}

\author{
Clayton H. Allen, Chairman
}

\section{Bolt Beranek and Nezman, Cambridge, Massachusetts 02138}

\section{Contributed Papers}

\begin{abstract}
AA1. Regulation of Vehicular Noise in Hawaii. J. C. Burgess, Department of Mechanical Engineering, University of Hawaii, Honolulu, Hawaii 96822.-A detailed Public Health Regulation entitled, "Vehicular Noise Control For Oahu," is awaiting signature by the Governor. The regulation applies to all vehicles and their auxiliary devices when operated on trafficways on the Island of Oahu. Separate objective standards are specified for "light" and "heavy" vehicles. Motorcycles are included with the light vehicles. For all vehicles, operational noise limits are specified in terms of sound level $A$, posted speed limit, and measurement distances between 20 and 50 $\mathrm{ft}$. For heavy vehicles, times of day and days of the week are also specified. Goals for the future are identified. An important feature of the regulation is the definition of an extensive permit system. Its function is to allow stringent standards to be set initially while providing for administrative flexibility to grant exceptions necessary in the public interest. Other important sections of the regulation concern exemptions, truck routes, mufflers, measurement, enforcement, and publication.
\end{abstract}

AA2. Subjective Study of Tire and Engine-Exhaust Noise. adnan Akay, William F. Reiter, Jr., and Haryey Cohen, Center for Acoustical Studies, North Carolina State University, Raleigh, Norlh Carolina 27607.-Effective enforcement of noise regulations governing ground transportation vehicles depends on a valid noise rating scale for monitoring the noises involved. Previous investigations have shown that the $\mathrm{dB} A$ rating is acceptable for tire noise and engine noise. This investigation was aimed at evaluating rating scales for comparison of tire and engine-exhaust noise. The method of paired comparisons was used to evaluate subjectively noise composed predominantly of truck tire noise compared to diesel engine-exhaust noise at three different vehicle and engine speeds. The tests were conducted in a simulated home environment. Recordings obtained under actual field conditions were used as stimuli and were presented at two different sound pressure levels to a total of 85 subjects in groups of 10 or fewer. The stimuli presented were rated objectively in PNdB, EPNdB, SAE Phons, $A$-weighted SPI, and according to the Stevens Mark VII criteria. A correlation analysis was carried out to determine the relative validity of the these ratings. [NASA is acknowledged for partial sponsorship of this work.]

AA3. Interior/Exterior Noise Levels of Over-the-Road Trucks. Thomas L. Quindry and William A. LEasure, JR., Applied Acoustics and Illumination Secion, Inslitute for Applied Technology, Nalional Bureaw of Standards, Washington, $D$. C. 20234. - Interior/exterior measurements were made on 15 trucks covering many makes, models, and drive-train combinations to provide an information base concerning vehicle interior noise levels for rulemaking by the Bureau of Motor 
Carrier Salety, Department of Transportation. Measurements were made $6 \mathrm{in}$. from the left and right ears of the drivers under various vehicle operational modes with windows open and closed. Stationary tests included: (a) low idle, (b) acceleration from low idle to maximum governed rpm with wide open throttle while in neutral gear; and (c) high idle. In addition to the acceleration and deceleration tests specified by SAE J366 (Exterior Sound Level for Heavy Trucks and Busses), an additional moving test was performed to simulate a truck starting up in city traffic. In order to study the effect of total truck noise on the community, simultaneous exterior measurements were made with an array of six microphones. The data obtained, as well as the test procedure and measurement techniques utilized, will be discussed.

\section{AA4. Nonlinear Effects in Helmholtz Resonator Mufflers for Internal Combustion Engines. A. J. Brammer, N. Olson, J. E. Piercy, And F. E. Toole, Physics Division, National Re- search Council, Ottawa, Canada.-The design of exhaust mufflers for internal combustion engines in practice tends to rely heavily on empirical art. One reason could be that the available theory is generally linear (hence suitable for low sound levels) and the levels inside the muffler are too high. We have chosen a simple case, one-stage Helmholtz resonator mufflers mounted on a small one-cylinder two-cycle engine, to test experimentally for nonlinear effects. Some configura- tions that are equivalent according to linear theory are found to produce insertion losses which differ by at least a factor of 2 , as measured in $\mathrm{dB} A$. Experimental evidence indicates the configuration in which tail and exhaust pipes are aligned is particularly ineffective because nonlinear jetting of the type analyzed previously for orifices by Ingard and his colleagues tends to bypass the volume.}

\section{AA5. Economics of Noise Control in New York State. AllaN} M. TEPLITzKY, Office of Environmental Affairs, Consolidaled Edison Co. of New York, Inc., New York, New York 10003.Department of Labor accupational noise-exposure standards will not protect all employees from hearing loss. For an occupational noise exposure of $90 \mathrm{~dB} A$ for $8 \mathrm{~h} /$ day for 10 years or more, the expected New York State Workmen's Compensation award per employee per year is estimated to be $\$ 25$. A comprehensive hearing conservation program to reduce an employer's liability for hearing loss could be financed by savings in potential compensation awards. Similarly, in plants where the occupational noise environment exceeds O.S.H.A. noise-exposure requirements, expenditures for engineering methods for noise control may be economically justified by savings in potential compensation payments. This paper reviews the methodology for determining the expected employee compensation award for occupational hearing loss and relates it to potential hearing conservation program costs.

AA6. Punch Press Noise, A Program for Analysis and Reduction. O. A. Shinaishin, General Electric Company, Corporate Research and Development, Schenectady, New York 12345.Occupational Safety and Health regulations are demanding the search for solutions to industrial noises in factories. Presses are among the more common and most noisy of the offending manufacturing machines. This paper discusses an approach to reducing the noise at the source which is the most desirable solution. Experimental and analytical work was done to isolate the separate sources of noise and their contribution to the over-all noise. Presses were instrumented to monitor the different events generating excitation forces, and to measure the vibration levels of significant parts. Analysis of the data from vibration and sound made possible the correlation between the over-all sound, the different events, and the vibrations of key parts in the press. Steps are outlined to change the character of forces and vibrating parts to reduce the noise.
Preliminary laboratory tests indicate that substantial noise reduction can be achieved once the diagnostics have been completed successfully.

AA7. Noise Reduction of a Residential Air Conditioner. Andrew F. Seybert, Cummins Engine Company, Columbus, Indiana 47201, AND Malcom J. Crocker, Ray W. Herrick Laboratories, School of Mechanical Engineering, Purdue University, Lafayette, Indiana 47907.-The major sources of noise in a residential air-conditioner were studied. SPL and acceleration spectra were measured in an anechoic room. The noise sources were found to be the condenser fan motor, the evaporator fan motor, and the compressor. Measurements revealed that the fan motors were vibrating predominantly at twice line frequency, $120 \mathrm{~Hz}$, and the vibration was being transmitted to the air-conidtioner panels. The research was concentrated on reducing this $120-\mathrm{Hz}$ hum. The effectiveness of the fan-motor-isolation systems was studied by varying the frequency of the current supplied to one motor. The motor acceleration and the SPL at twice line frequency were both monitored. The acceleration frequency plot revealed a fanmotor-isolation system resonance frequency close to the operating frequency of $120 \mathrm{~Hz}$. By reducing the stiffness of the mounting brackets of each of the fan motors, the SPL at $120 \mathrm{~Hz}$ was reduced by $12.5 \mathrm{~dB}$. [Work supported by American Standard.]

AA8. Automatic Control of Transformer Noise by Synthesizing Directivity. KEN'ITI KIDO, Research Institute of Electrical Communication, Tohoku University, Sendai, Japan.-The noise of outdoor transformers has been controlled chiefly by acoustic screen, but the effect of the screen has not been so large. On the other hand, some efforts to make a transformer with low noise level have been carried out and the radiated noise level from newly designed low noise transformer has been largely decreased by the vibration insulation between the core and the outer case. But the cost of the transformer has increased. The idea of controlling the noise with some additional sound sources had been proposed as an acceptable method, which is adopted in this paper. The sound field around the transformer must be controlled by the adjustment of the amplitudes and the phases of the additional sound sources. But how to adjust them is so difficult that there have not yet been practical results. The proper adjustment of the additional sound sources is difficult because the conditions of the sound field and the power source may change at times. Therefore, it is necessary to control automatically the amplitudes and the phases of the additional sound sources. The author has developed the practical method of automatic control of the noise and proved the effectiveness of the controlling method. The outline of the fundamental considerations and the successful results of the field experiments are described in this paper with the explanation of directivity synthesis, problems of stability, and the controlling procedure, especially the successive adjustment method.

AA9. Analysis of Noise Generation in Industrial Wood Planers. John S. Stewart and F. D. HART, Center for Acoustical Studies, Department of Mechanical and Aerospace Engineering, North Carolina State University, Raleigh, North Carolina 27607 . - The primary sources of noise from industrial wood planers are associated with board radiation and aerodynamic phenomena. The factors that control board radiation have been studied analytically and experimentally. The parameters considered include board width, length, thickness, and wood species. A model for determining the sound power emitted because of board radiation is developed, and theoretical results are compared with experimental data. Board width is shown to be the most important board geometric parameter 
in noise radiation. [NASA and Newman Machine Company are acknowledged for partial sponsorship of this work.]

AA10. Control of Noise Sources on Sea-Going Tow Boats. K. D. Harford, A. J. Price, and T. E. Siddon, Acoustical Engineering, Suile 3- 690 Number Three Road, Richmond, British Columbia, Canada.-The paper describes noise-control studies on a number of commercial tow-boats operating on the North-Pacific coast. Noise levels in these small vessels often exceed $80 \mathrm{~dB} A$ in the sleeping accommodation areas. Crew may be continuously exposed to these high levels of noise for periods of several days. A variety of diagnostic techniques have facilitated the separation and localization of sources of structure-borne and air-borne sound. These include the use of artificial sources, spectral and cross-correlation analysis, vibration analysis, and statistical encrgy methods, together with the less sophisticated trial and elimination approach. On most boats, structure-borne vibration made the greatest contribution to noise levels in occupied spaces. The high noise levels were directly attributed to one or more mechanical deficiencies on each boat. Such deficiencies included faulty bulkhead design, inadequate acoustic isolation of crew quarters, and, principally, the widespread practice of mounting the engine, gear box, and ancillary components rigidly to the main structural elements of the vessel. Implementation of noise-control recommendations resulted in significant noise reduction on several vessels. Quantitative results for isolation of both engines and occupied spaces are presented.

Thursday, 20 April 1972

Terrace Room, 9:00 A.M.

\title{
Session BB. Symposium on Deafness
}

\author{
LoIs L. ElliotT, Chairman
}

Office of Education, Department of Health, Education and Welfare, Washington, D. C. This session is given in memory of Flizabeth Guild.

\section{Invited Papers}

BB1. The World of Deafness-As Viewed by An Insider. MALCOM NoRwood, Bureau of Education for the Handicapped, U.S. Office of Education, Washington, D. C. 20202.-A first-person account of the experiences and problems encountered in a world of silence. Described are a personal reaction to the loss of hearing and the reaction of the family; educational experiences; social life; methods of communication; and problems constantly laced in a competitive professional world.

BB2. Genetic Features of Deafness. Kennetr S. Brown, National Institutes of Health, Bethesda, Maryland 20014. - The pattern of hearing loss of children in schools for the deaf provides little information regarding etiology, but it is possible to evaluate the relative contribution of several etiologic factors by study of medical histories and family records. Traits such as thyroid or pigment anomalies can be used to identify particular genetic defects in a small proportion of deaf children; however, family data suggest that over half of all cases are the result of inheritance. Although most of the responsible genes are not now recognizable, pedigree data give excellent fit to simple genetic nodels. Thus we are able to estimate the minimum number of genes causing deafness and predict risks of deafness in offspring. Using experimental animals with patterns of hereditary deafness similar to man, we can observe development and anatomy which suggest important directions for the study of deaf children.

BB3. Anatomical Correlates of Deafness. CATHERINE A. SMlTh, Department of Otolaryngology, University of Oregon Medical School, Portland, Oregon 97201.- Structural defects associated with deafness may be divided into two classes. The first class is associated with structures of the external and middle ear with the result that conduction of sound is impeded. Deformities of the external auditory canal and the ossicles may be hereditary and present at birth. Middle-ear infections, otosclerosis, etc., acquired later may alter normal ossicular clain function. If properly diagnosed, for the most part these can be repaired by surgery. In the second class, the pathology is restricted to the inner ear. If the defect is congenital, it may have been inherited, or occurred "in utero" such as with rubella infections. After birth, an individual may be exposed to any number of situations which cause destruction of the hair cells and the cochlear nerve fibers. If the loss is extensive enough, a defective receptor organ results, with partial or complete deaf ness of the individual. It is not clear at present precisely what percentage of hair cells and nerve fibers are necessary for adequate social communication, but there is no replacement of degenerated receptor cells or sensory nerve fibers.

BB4. Amelioration of Deafness. Edwin W. MARTIN, Associate Commissioner of Education, Bureau of Education for the Handicapped, $U$. S. Office of Education, Washington, D. C. 20202, ArthuR F. Niemoeller, Central Institute for the Deaf, St. Louis, Missouri and Kenneth N. Stevens, Massachusetts Institute of Technology, Cambridge, Massachusetts.-Although medical advances will help prevent deaf $n$ ess and engineering developments may provide auxiliary resources, the primary responsibility for ameliorating this handicapping condition, particularly for children, rests on education. Available evidence suggests that the age of beginning educational intervention, including auditory amplification, language and speech development, and parent guidance, is extremely important-the earlier the better. Early education also requires procedures for identification of deafness. Some 
States have now legislated programs for children younger than five years, but much more remains to be accomplished.

\title{
Session CC. Speech Communication V : Acoustical Properties of Pathological Speech
}

\author{
George W. Hughes, Chairman
}

School of Electrical Engineering, Purdue University, Lafayette, Indiana 47907

Invited Papers (30 minutes)

CC1. Acoustical Properties of Alaryngeal Speech. B. Weinberg, Speech Research Laboratory, Indiana University Medical Center, Indianapolis, Indiana 46202.-This paper will review recent findings coming from a research program in which the acoustic properties of various types of alaryngeal speech were examined. The fundamental frequency, rate, phonation time, long-term spectral, and vowelformant frequency characteristics of male and female esophageal talkers were measured in an attempt to provide information about the physical properties of esophageal speech and to find correlates to listeners' judgments of the sex of these speakers' voices. Significant sex differences were found for some of the physical measures. These studies show that close relationships can be found between the acoustical and perceptual spaces of esophageal voices. In other experiments, the acoustical properties of two other types of alaryngeal speech-buccal and pharyngeal speech-were measured in an attempt to evaluate their potential use as primary methods of alaryngeal speech. Studies of the mechanism of phonation, fundamental frequency, phonation time, rate, intelligibility, and long-time spectral characteristics of buccal and pharyngeal speech were completed. The results of these experiments support the hypothesis that both buccal and pharyngeal speech do not represent efficient or practical primary methods of alaryngeal speech.
\end{abstract}

CC2. Laryngeal-Disorder Detection through Speech Analysis. T. H. Crystal, SIGNATRON, Inc., Lexington, Massachusetls 02173 . - This talk reports on a study into the feasibility of using automatic voice analysis for the detection of laryngeal disorder. Over 200 normal and pathological subjects were examined and had their voices recorded at the Massachusetts Eye and Ear Infirmary. The first stage of the automatic analysis was a highly accurate peak detection and pitch tracking on a throat microphone signal [Crystal, T. H., and C. L. Jackson (1970), J. Acoust. Soc. Amer. 48, 118(A)]. Lieberman's pitch perturbation technique [P. Lieberman (1963). J. Acoust. Soc. Amer. 35, 334-353] was extended to amplitudes. Our analysis was made on "jitter" defined as the difference between smoothed data and actual data. The normalized mean-square jitter was then used as an index of vocal quality. A set of such indices, obtained by using different smoothing algorithms, was used in a multivariate discriminant analysis of the data. Linear discrimination on the training data allowed the separation of $83 \%$ of the male pathologicals when the decision threshold was set to misclassify less than $7 \%$ of the male normals. Less separation was obtained with females. This performance has also been compared to the effectiveness of a voice-evaluation procedure, which was specially introduced into the physician's examination. A review of the procedures and data together with the rate of incidence of laryngeal disorder suggests certain directions for the continuation of this work. [The original work was supported under the Neurological and Sensory Disease Control Program, U. S. Public Health Service, whose personnel contributed to the project.]

CC3. Acoustic and Perceptual Characteristics of the Speech of Deaf Children. Harry LevitT, Doctoral Program in Speech, The Cily University of New York, Graduate Division, 33 West 42 Street, New York, New York 10036.- The speech of deaf children differs from that of normal children in many ways. There are articulatory errors such as omission of consonants, insertion of adventitious sounds (typically between syllables and in consonant clusters), substitutions of one phoneme for another, moderate 
distortions in which the intended phoneme is still recognizable, and severe distortions in which there is no recognizable phoneme. In addition to articulatory errors involving individual phonemes, there are suprasegmental errors such as incorrect rhythm, stress, intonation, phrasing, and errars involving other prosodic features. There are also problems of voice quality such as excessive harshness, breathiness, nasality, and problems of pitch, i.e., average pitch too high, too low, or excessively variable. Acoustic measurements correlate reasonably well with these perceptual observations. On average, the fundamental frequency contours of deaf children tend to be higher than for normal children of the same age and sex, but there are notable exceptions in which the fundamental frequency contour is very low, or exhibits sudden changes from one extreme to the other. Formant frequencies during vowel sounds are similar to those for normal children, but are biased towards values typical of the schwa vowel. Substitution of the schwa for other vowels is a common error. Experiments involving measurements on other acoustic parameters, such as formant transitions and fricative spectra are currently in progress.

\section{3:30}

CC4. An Acoustic and Electromyographic Investigation of Consonant-Vowel Transitions in the Speech of Deaf Adults. H. B. Rothman, Communicalion Sciences Laboratory, Universily of Florida, Gainesville, Florida 32601.-An acoustic and electromyographic investigation of the speech of normal-hearing and deaf speakers attempted to answer questions concerning formant transitions, coarticulation, and neutralization of vowels in the speech of deaf adults. In order to accomplish this, EMG and acoustic recordings were made simultaneously as each subject read a constant phrase into which a variable key word was embedded. The deaf speakers chosen for this study were judged to be above average in intelligibility for deaf talkers with a profound bilateral hearing loss of early onset. Acoustical analysis was performed by making wide-band spectrograms of selected recorded stimulus items. The amplitude display of the sonograph was used for relating electromyographic events to acoustic events in time. The results of this study showed that the transitions of deaf speakers had a restricted range of movement and a slower rate of movement than those of normal speakers. The speech of the deaf shows relatively small coarticulation effects, their vowels tend to be neutralized regardless of the context, and they tend to begin an articulatory sequence in a similar manner regardless of the context. The deaf speakers also exhibited difficulty coordinating articulation with phonation and their articulatory behavior showed greater variability than did the articulatory behavior of the normal speakers. The discussion of the results will indicate their implications for speech training of the deaf.

\section{4:00}

CC5. Acoustic Measures of Nasalization and Nasality. Martin F. Schwartz, Department of Speech, Temple University, Philadelphia, Pennsylvania 19122. - In this paper a description is given of four of the primary acoustic features that have been found to be associated with the nasalization of vowels. Comparative normal-nasal spectra are portrayed for several vowels, and explanations to account for each of the prominent within-vowel differences are offered. In addition, other evidence is presented which is used to suggest that each of the primary acoustic features of nasalization may also be the result of factors quite unrelated to nasal coupling. These factors, manifestations of typical normal variations, are used to develop the thesis that it is not readily possible to isolate the acoustic features of nasalization within a given vowel spectrum. The implications of this thesis are then manifest in a questioning of the validity of the term, nasality.

\section{4:30}

CC6. Some Acoustic Aspects of Stuttered Speech. J. G. AGnello, Department of Speech Science, Universily of Cincinnali, Cincinnati, Ohio 45221, AND M. E. WINGATE, State University of New York, Buffalo, New York 14202. - Three extensive studies have been conducted on the acoustic features of stuttered speech. One study examined the temporal properties and formants of speech having various degrees of fluency. Another study reports on the coarticulation patterns across two languages (ItalianEnglish) of stutterers and nonstutterers. The third study (discussed more extensively) is on the spectrographic patterns of simultaneous intraoral air pressure and formant patterns of stutterers and nonstutterers. Stutterers and normal speakers produced a series of syllables of $/ \mathrm{pa}, \mathrm{ba}, \mathrm{ap}, \mathrm{ab}$, apa, aba/ and some short phrases. Simultaneous measures of intraoral air pressure and formant patterns of these utterances reflected the transitional features of labial release to glottal onset patterns, e.g., plosive to vowel. Also, vowel and plosive measurements reflected the manner of glottal termination followed by labial closure. Standard spectrographic tracings illustrate only the acoustic events of speech and pose certain problems of accurate temporal measurements for stutterers, since a great deal of their speech efforts are essentially nonacoustical. A system is presented that yields a more discrete pattern of the articulatory events in terms of formant patterns and intraoral air pressure. The transitional features in the fluent and nonfluent production of syllables uttered by stutterers and nonstutterers will be illustrated. The difference is shown to be within the transitional aspects of segmental units. The transitional features within the syllabic structure of the stuttered speech point to a disruption of coarticulated events. This transitional failure is the phenomenal core of stuttering. Implications will be discussed, particularly that voice control is the core element in stuttering behavior. 
Session DD. Bull Session on Cavitation and Its Uses

\author{
Wesley L. Nyborg, Moderator \\ University of Vermont, Burlington, Vermont
}

\title{
Sponsored jointly by the Technical Committees on Physical and Engineering Acoustics
}

There will be opportunity for informal discussion on cavitation theory and practice. Topics will include standardization of ultrasonic cleaners; relationship of cavitation theory to practical situations; possibilities for design of cavitation fields; and biological and medical applications of sonic cavitation.

\section{Session EE. Shock and Vibration IV : Dynamic Characteristics of Anthropomorphic Dummies and Auto Crash Studies}

\author{
Henning E. von Gierke, Chairman \\ Department of the Air Force, 6570th A erospace Medical Research Laboratory, \\ Wright-Patterson Air Force Base, Ohio 45433
}

\begin{abstract}
Invited Papers
EE1. Crash Survivability. Charles Y. Warner, National Highway Traffic Safety Administration, Department of Transportation, W'ashington, D. C. 20590.-The starting point for automative crashworthiness design must be the tolerance of the human biological system to impact. For obvious reasons, human tolerance to impact cannot be evaluated by a simple test of ultimate strength. The human body has an infinite number of degrees of freedom, and many modes of loading which pose threats of injury. Further, no samples of actual material are available for destructive testing. Present design information on human tolerance is a composite of extrapolations from accidental impacts, human experiments at voluntary levels, cadaver experiments, and animal exposures at injury and fatal levels. Although a large body of experimental data has been accumulated, it must be carefully interpreted if valid design decisions are to be reached. Anthropometric dummies enter into the design process at two levels. First, dummies can contribute to knowledge of occupant crash dynamics, at least up to the instant of second collision. (The contribution thereafter is greatly dependent upon the degree of sophistication of the dummy.) Second, the dummy may be used to evaluate the performance of a given design. Of course, results of any testing with dummies depend upon the realism of dummy performance for the specific hardware and the mode of impact being investigated. This presentation outlines one program for determining automobile crash survivability criteria. Human tolerance is estimated by volunteer and animal tests of several restraint systems. The interaction of the vehicle structure, restraint system, and occupant are demonstrated by the results of prototype vehicle crash tests.
\end{abstract}

EE2. Dynamic Characteristics of the Crash Victim - Comparison of Experimental Results on Anthropomorphic Dummies with a Three-Dimensional Computer Simulation. J. A. Bartz and R. A. Przialı, Cornell Aeronautical Laboratory, Inc., Buffalo. New York 14421.-A mathematical model of the crash victim, either vehicle occupant or pedestrian, in a three-dimensional crash environment has been developed and validated at CAL. The result of this research is a functional engineering tool for parametric investigations of the dynamic behavior of the creash victim and for design of related experiments. The digital computer simulation includes a dynamics model with $\mathbf{4 0}$ degrees of freedom and a contact model that generates contact forces from vehicle surfaces and between body segments of the crash victim, and restraint forces from belts and inflatable restraints (air bags). Assumptions regarding the dynamic characteristics of the crash victim model are reviewed in detail. Experimental validation consisted of dummy response measurements in a series of accelerator sled tests simulating 20 and $30 \mathrm{mph}$ barrier crashes. The experimental results include dummy kinematics, head and chest accelerations, and restraint forces. In another research effort, CAL had conducted an experimental program to assess the dynamic performance of the state-of-the-art anthropomorphic devices. Two dummies of each of two manufacturers were tested in each of five configurations on an accelerator sled simulating a $30 \mathrm{mph}$ barrier crash. Three replications of each test were made to provide a measure of 
the reproducibility of the results. A brief summary of the results is made to indicate the variation in response between the dummies of the two manufacturers in the different configurations and the reproducibility of the results obtained.

EE3. Dynamic Testing of Biological Materials. James McElhaney and John Melvin, Highway Safety Research Institute, The University of Michigan, Ann Arbor, Michigan 48105.-Because the mechanical properties of biological materials exhibit strong rate dependence, special care must be exercised in their measurement. This paper describes a variety of measurement techniques to determine the dynamic mechanical properties of hard and soft tissues. Results of these tests on bone, scalp, brain, and dura are presented. Viscoelastic models, modeling techniques, and difficulties are discussed for these materials. Materials from both human and subhuman primates have been studied and comparisons made. The effect of post-mortem test times and storage and specimen preparation methods is discussed.

EE4. Evaluating Car Safety by Computer-Simulated Crashes. R. J. Melosh, Philco-Ford Corporation, Palo Alto, California 94303.-Computer simulation of car crashes prescribed for safety assurance offers the prospect of relatively precisely controlled, densely instrumented, low cost testing. The computer environment engenders short lead times, extensive problem descriptive data, and automatic data reduction. This presentation describes the principal concepts of mathematical simulation of the nonlinear dynamic characteristics of an automobile structure. It shows that the number of calculations are three orders of magnitude greater than for a comparable linear systen, illustrates the importance of developing a special purpose computer code, shows the effectiveness of automatic error control, and expounds on the complexity of validating the numerical analog. Figures showing simulation results demonstrate the fidelity with which time dependent, large deflection, and finite strain behavior are emulated for simple problems. Physical and computer accelerations for a head-on crash configuration are compared.

EE5. Crashworthiness-in Perspective. Richard A. Wilson, Safety Research and Development Laboratory, General Motors Proving Ground, Milford, Michigan 48042.-Crashworthiness for automotive vehicles concerns more than how the fenders bend. The total system is involved from the vehicle's structure to the occupant's restraint. Field accident data must be used to establish the mix of variables required to provide an acceptable level of crashworthiness. Vehicle weight, structural stiffness, acceleration pulse, type of occupant restraint, degree of passenger compartment intrusion-all must be balanced and put into proper perspective. Experimental Safety Vehicles (ESVs) can be useful exercises in designing for arbitrarily set crashworthiness requirements, but they are not necessarily examples of designs that balance all vehicle requirements.

Thursday, 20 ApriL 1972

GOVERNOR's ROOM, 2 :00 P.M.

\title{
Session FF. Underwater Sound Systems
}

\author{
Thaddeus G. Bell, Chairman \\ Naval Underwater Systems Center, New London, Connecticut 06320
}

Invited Paper (30 minutes)

FF1. The Development and Use of a Long-Range Side-Scan Sonar. J. S. M. RuSBY, National Institule of Oceanography, Wormley, Godalming, Surrey, England.- The paper describes the construction and use of a long-range side-scan sonar, which was designed by the National Institute of Oceanography to view the ocean floor out to a maximum range of 12 miles. The sonar array is mounted in a streamlined fiberglass vehicle towed at $300 \mathrm{ft}$ at a speed of $7 \mathrm{kt} .6 .5-\mathrm{kHz}_{2} \mathrm{CW}$ or FM pulses can be radiated at $50 \mathrm{~kW}$ in a beam $2 \frac{1}{2}^{\circ}$ by $10^{\circ}$ in the horizontal and vertical planes, respectively. The returned FM signals can be processed in real time on either a digital or analog correlator. The sonar has been in use since 1969; in 1971, it surveyed parts of the Mediterranean Ridge and the Azores-Gibraltar Ridge, and a region of deep trenches near the Mid-Atlantic Ridge, in all covering 35000 square miles. During the year, an exploratory long-range survey was also made of a Scottish commercial fishery. 


\section{Contributed Papers (12 minutes)}

\section{2:30}

FF2. Sources of Probability in Predicting Passive Sonar Performance. F. A. ANoRews, Department of Electrical Engineering, The Catholic University of American, Washington, D. C. 20017.-There are three sources of probabilistic statements found in predicting passive sonar performance. These are (1) the detection process for a single observation time $T$; (2) the sequence of multiple observations carried out by a sonar operator resulting in $N$ independent observations; and (3) the availability of a given level of input signal to noise $(\mathrm{S} / \mathrm{N}$ ) ratio. The first two considerations result in a conditional detection and false-alarm probability given a fixed value of input $S / N$ ratio. Recognition differential is seen not as the value of input $S / N$ ratio required for detection probability $50 \%$ but rather the minimum value of $S / \mathrm{N}$ ratio that will trigger the sequential observation process and lead to a detection probability near unity and false-alarm probability near zero. The third consideration results in a probability density distribution for input $\mathrm{S} / \mathrm{N}$ ratio because one wishes to predict detection for an ensemble of targets, propagation loss conditions, sea states, and sonar operators. The probability of detection for a given variance and expected value of input $S / N$ ratio and given value of recognition differential is seen to be the integrated product of the first two probabilities times the third. The probability of a false alarm in passive sonar operation is negligibly low'because of the sequential observation process. A sample problem for aural detection and for detection by time-bearing plot will be presented.

\section{$2: 45$}

FF3. Advantages of a Two-Transducer Frequency Analysis Technique to Characterize Discontinuities. Laszlo AdLer, Department of Physics, University of Tennessee, Knoxville, Tennessee 37914, AND H. L. WHALEY, Oak Ridge National Laboratory, Oak Ridge, Tennessee 37830.-In an earlier paper [J. Acoust. Soc. Amer. 48, 102(A) (1970)], we have shown that the frequency analysis of a broad-band ultrasonic echo may be used to determine the size and orientation of flat circular reflectors. A single transducer was used as both source and receiver. The one-transducer technique is not completely satisfactory for the case of discontinuities having both random shape and orientation. Recently, we have used a second transducer to receive ultransonic pulses scattered by a reflector. Defects in materials were simulated by irregularly shaped metal shims suspended with random orientation in water. The received frequency spectrum when analyzed shows characteristic maxima and minima, which depend on the shape, size, and orientation of the reflectors. A model-which assumes an interference pattern formation of the scattered spherical wavelets at the receiver-relates the spacing between frequency maxima and the positions of the two transducers to the size and orientation of the "discontinuities." The measurement and the analysis is greatly simplified with the use of the second transducer, and size determination of the discontinuities can usually be made within 10\%-20\%. [Research sponsored by the U. S. Atomic Energy Commission under contract with the Union Carbide Corporation.]

$$
\text { 3:00 }
$$

FF4. Detection of Sampled Signals. R. O. RowlanDs, Ordnance Research Laboratory, The Pennsylvania State University. State College, Pennsylvania 16801.-In correlating samples of a bandpass signal against a reference, two quadrature sets of the input samples are of ten used to ensure that a high output is obtained at zero delay. It is shown that this duplication is unnecessary as even if the single sample set represents the quadrature version of the reference, at least two samples of the output signal will occur that can be processed further to give an output within $1 \mathrm{~dB}$ of the theoretical peak.

\section{$3: 15$}

FF5. Code Design for a Transmit Beam Scanning Sonar. J. M. Ross, Defence Research Establishment Allantic, Dartmouth, Nova Scotia, Canada.-A scanned parametric transmitter is being constructed at Birmingham University. The receiver associated with the system is tuned to the difference frequency obtained from the interaction in water of the two transmitted primary frequencies. The transmitter scans a sector about $15^{\circ}$ in width with a beam approximately $1.5^{\circ}$ wide. One means of coding the transmitted beam is by sending a different code word into each subsector $\left(1.5^{\circ}\right)$ of the scanned sector. The code words considered each consist of a sequence of $\boldsymbol{A}$ pulses, each pulse of which can have one of $N$ carrier frequencies. The target bearing is identified by correctly identifying the received code word. A two-threshold receiver provides better reduction of ambiguities caused from overlapping signal bandwidths than a one-threshold receiver. One means of reducing false alarms due to ambiguities is to rotate code words with each transmission. Bearing resolution can be improved by scanning the transmit beam less than one subsector beamwidth for each code word transmitted.

$$
\text { 3:30 }
$$

FF6. A Method for High-Speed Calculation of the Exact Solution for the Pressure in the Nearfield of a Circular Baffled Piston. J. G. Willette and J. C. Lockwood, Applied Research Laboratories, The University of Texas at Austin, Austin, Texas 78712.-A high-speed method has been developed for calculating from the exact integral solution the pressure at each point in the nearfield of a sinusoidally excited circular piston in an infinite baffle. Previously the exact nearfield pressure has been calculated by means of a double numerical integration over all source points on the piston face. The phase of the signal arriving at a field point from each point on the piston must be taken into account precisely. One investigation in which the double integration was employed is that of Zemanek, who published three-dimensional and contour plots of data obtained by this method [J. Acoust. Soc. Amer. 49, 181-191 (1971)]. We have reduced the calculation to a single numerical integration by starting with the known exact solution for the field produced by impulse motion of the piston. For each field point, we compute, at the frequency of interest, the Fourier transform of the impulse response. Thus, we obtain the transfer function, a complex quantity that, when expressed in polar form, gives us the amplitude and phase of the pressure variation. We have made three-dimensional and contour plots that compare favorably with those published by Zemanek. The high speed of our method makes possible the efficient calculation of the difference frequency source function. (See J. G. Willette, Paper F9 of this meeting.) [This work was sponsored by the Office of Naval Research.]

\section{$3: 45$}

FF7. Theoretical Design of Nearfield Calibration Arrays of Arbitrary Configuration. A. L. VAN BuREN, Naval Research Laboratory, Washington, D. C. 20390.-The Trott nearfield calibration array (NFCA) is an experimentally proven concept for determining the farfield acoustic radiation of large underwater transducers from measurements in the nearfield. 
Existing successful NFCAs are large planar configurations of small reciprocal transducers whose responses are amplitude shaded so that the NFCA as a source produces a nearly uniform plane wave directed normal to the NFCA throughout the volume to be occupied by the unknown transducer. Trott obtained shading coefficients that are effective over a wide frequency range by the use of semiempirical methods that are applicable to linear or planar arrays. In this paper, a rigorous derivation of the NFCA reciprocity principle is presented for the first time. The results allow for both amplitude and phase shading and are applicable to NFCA configurations other than linear or planar. A numerical technique based on this principle is described that produces an optimum set of shading coefficients for an arbitrary NFCA configuration with a prescribed plane-wave direction, volume, and frequency domain. As an example, the design of a cylindrical NFCA suitable for calibrating an enclosed cylindrical transducer without mechanical rotation is presented and discussed.

\section{4:00}

FF8. Spectrum Measurement in the Field of an Ertended Source. Donald A. MURPhy, Hughes Aircraft Company, Fullerton, California 92634.-The spectrum at points in the field of coherent line sources and coherent line arrays was calculated. Large fluctuations exist in the farfield due to the directivity pattern for a fixed length source except on the main lobe of the source directivity pattern. When the length of the source is inversely proportional to the frequency, the spectrum is undistorted at all points in the farfield. In the nearfield, however, the spectrum is distorted even for the on-axis case for a fixed length source. It is less distorted than in the farfield for points outside the main lobe of the source. For a source whose length is inversely proportional to frequency, the high frequencies are in the farfield and the low frequencies are in the nearfield. The spectrum contains a lowfrequency peak whose frequency depends upon the distance from the source where the measurements are made. Errors of estimating radiated pressure levels by measuring in this nearfield and extrapolating by inverse distance spreading can be quite large.

FF9. The Directivity of Sonar Receiving Arrays: The NonOmnidirectional Case. William C. QUEen, Department of Mathematics, University of South Carolina, Columbia, South Carolina 29208. - The limiting case of a continuously distributed array has long been recognized as a useful aid in the design and study of sonar receiving arrays consisting of a large number of elements. In a paper appearing in 1963 , Anderson and Munson utilized this approach to determine the directional characteristics of spherical arrays, and more recently (1970), W. C. Queen pursued this idea further to include more general configurations. In all these analyses, the hydrophones were considered to be omnidirectional. In this present paper, the more general situation of a continuously distributed array of nonomnidirectional elements is considered, and results are given for the special case of a spherical array whose elements exhibit hypercardioid response patterns.

$$
4: 30
$$

FF10. Closed-Form Solution for the Radiation from a Continuous End-Fire Line Array. H. O. Berktay and J. A Shooter, Applied Research Laboratories, The University of Texas at Austin, Austin, Texas 78712.-An "exact" closedform solution for the acoustic field from a continuous end-fire line array is presented. Interactions between the array elements are ignored, but no approximations are made regarding the position of the field point. (This model lends itself particularly well to the study of the response of parametric receivers.) The results are presented in the form of beam patterns as a function of range. It is shown that the range where the farfield response is obtained depends only on the length of the array and that the beam patterns within the nearfield can be substantially more narrow than the farfield beam patterns. [This work was sponsored by the Office of Naval Research.]

Session GG. Noise III : Jet and Propeller Noise

\author{
H. S. Ribner, Chairman
}

Institute of Aerospace Studies, University of Toronto, Toronto, Canada

\title{
Contributed Papers (12 minutes)
}

\section{2:00}

GG1. Basic Directivity of Jet Noise with Improved Correction for Refraction. H. S. Ribner, G. R. MACGREGor, AND H. LAM, Institute for Aerospace Studies, University of Toronto, Toronto, Canada.-In earlier work of Grande, experimental jet-noise polar plots were corrected for refraction (by experiment) and for convection (by theory) to yield the experimental basic directivity in narrow frequency bands. The experimental correction for refraction at each frequency was obtained from the difference in the directional patterns of a point source placed in the air jet with jet off and jet on. Before refraction, this point source will produce a spherical directional pattern, but the narrow-band jet noise will have an elongated pattern owing to convection. It has been realized belatedly that the refractive change in intensity at corresponding angles cannot be taken as the same for the two different initial polar patterns: there is some violation of energy conservation. To avoid this error, later unpublished measurements by MacGregor have been reworked by Lam, incorporating a correction to the refraction effect measured with the point source. The correction was specified by an assumed functional form as a polar plot; the amplitude was then dictated by the requirement of conservation of energy. The measured jet noise data, processed along the lines of Grande, then leads to much improved agreement of experiment and theory. [Research supported by Air Force Office of Scientific Research.]

$$
2: 15
$$

GG2. Reduction of Peak Jet Noise using Jet Refraction. P. J. MORRIS, W. Richarz, AND H. S. RIBNER, Institute of 
Aerospace Studies, University of Toronto, Ontario.-The directivity pattern of sound generated by a subsonic jet exhibits a peak at angles between $15^{\circ}$ and $45^{\circ}$ to the jet axis decreasing to a minimum on the axis. Theoretical and experimental investigations have previously demonstrated that this minimum is due to refraction of sound by the jet flow. The present experimental study considers the effect of the introduction of a second jet of smaller exit area near to the main jet. It is shown that the second jet imposes its own heartshaped directivity pattern on that of the first jet. Alteration of the angle between the two jets leads to a reduction in the peak sound level. Spatial distributions and spectra employing unfiltered and filtered measurements are presented for changes in the temperature ratio of the two jets and their geometrical configuration.

\section{$2: 30$}

GG3. A Theory of Excess Jet Noise. D. G. CRIGHTON, Mathematics Department, Imperial College, London, England.Uniform subsonic jet flow issues from a semiinfinite circular duct, and undergoes a spatial instability. The sound field resulting from coupling of the instability to the duct is calculated. It is shown that the intensity of this "lip-noise" varies with speed $U$ and direction $\theta$ from the exhaust according to $I \propto U^{6} \sin ^{4} \theta / 2$, and that the maximum acoustic efficiency of this process is of order $10^{-5} M^{3}$. The spectrum peaks at a frequency rather higher than that characteristic of jet mixing noise, and the sound power is only slightly reduced by forward motion of the duct at typical aircraft landing speeds. These features offer an explanation of the so-called "excess noise" phenomenon, present in subsonic and supersonic jets alike, and consisting of substantial increases in forward arc noise over the levels predicted by Lighthill's theory of jet mixing noise. [Work supported by NGTE, Pyestock, England.]

\section{$2: 45$}

GG4. Further Studies of the Aeroacoustics of Jets Perturbed by Screens. Roger E. A. Arndt, Nam C. Tran, and Galen L. BAREFOOT, Department of Aerospace Engineering, Pennsylvania Stale Universily, University Park, Pennsylvania 16802.It is observed that the placement of a screen across the exit plane of a jet nozzle results in a substantial reduction in noise intensity over what would be realized with an equivalent unperturbed jet. In the past, the general features of this phenomenon were analyzed experimentally utilizing both farfield pressure measurements and velocity (hot-wire) measurements. This investigation has been extended to include the measurement of nearfield pressure along with a more extensive program of hot-wire measurements. Nearfield pressure measurements were made with a static pressure probe developed previously. These data were used to determine the distribution of sound sources in the screen perturbed jet. The results obtained from nearfield pressure measurements gave comparable information about the effect of the screen on the acoustics sources as was obtained with hot-wire measurements and theory. Extensive hot wire data indicate that there is a major change in the characteristics of the flow field of a screen perturbed jet. The potential core in the mixing region extends farther downstream and turbulent intensities are reduced to a lower level. The strength of the quadrupole sources (assumed proportional to Reynolds stresses) has changed from a maximum value of 0.011 to less than 0.008 near the center of the mixing region. Theory indicates that the acoustic power is also proportional to the fifth power of the longitudinal scale of the flow. Through correlation techniques, a reduction in the iength scale of the perturbed jet is observed. The results of the nearfield study are correlated with farfield observations.
3:00

GG5. Saturn Systems Holddown Acoustic Efficiency and Normalized Acoustic Power Spectrum. D. W. Gllbert, Teledyne Brown Engineering Company, Huntsville, Alabama 35807.- Saturn systems field acoustic data are used to derive mid- and farfield prediction parameters for rocket engine noise. The data were attained during Saturn vehicle launches at the Kennedy Space Flight Center. The data base is a sorted set of acoustic data measured during the period 1961 through 1971 for Saturn system launches SA-1 through AS-509. The model assumes hemispherical radiation from a simple source located at the intersection of the longitudinal axis of each booster and the engine exit plane. The model parameters are evaluated only during vehicle holddown. The acoustic normalized power spectrum and efficiency for each system are isolated as a composite from the data using linear numerical methods. The specific definitions of each allows separation. The resulting power spectra are nondimensionalized as a function of rocket engine parameters. The nondimensional Saturn system acoustic spectrum and efficiencies are compared as a function of Strouhal number with power spectra from other systems. [This analysis has been funded in part under contract under the cognizance of the George $C$. Marshall Space Flight Center.]

\section{$3: 15$}

GG6. Predicted Acoustic and Aerodynamic Noise Environments for the Reusable Space Shuttle Orbiter. H. Himelblau, Space Division, North American Rockwell Corporation, Downey, California 90241 .- Aeroacoustic noise generated by the proposed shuttle vehicle was predicted for various orbiter locations. Over-all SPL of 162-163 dB was calculated for shuttle liftoff, using Saturn V liftoff data and a scaling relationship based on the Eldred method. Over-all SPL of $167 \mathrm{~dB}$ was determined for the flow interference region between the mated booster and orbiter during the $q_{\max }$ period of ascent, using wind tunnel data obtained by Coe, Dods, and Robinson and flight data measured on Titan IIIC. Aerodynamic noise of $158 \mathrm{~dB}$ was predicted during ascent for regions immediately before and behind the cockpit using wind tunnel data provided by Coe and by Stevens, and for the mid- and aft fuselage using procedures developed by Bies and by Ailman, as well as Saturn V flight data. Maximum entry levels of 139-144 dB were estimated using separate procedures developed by Ailman, Bies, and Heller and Holmes.

\section{$3: 30$}

GG7. Predicted Vibration and Shock Environments for the Reusable Space Shuttle Orbiter. H. Himel Blau, Space Division, North American Rockwell Corporation, Downey, California 90241.--Structural vibration response to aeroacoustic noise, engine-induced mechanically transmitted vibration, and mechanical shock were predicted for various orbiter locations of the proposed shuttle vehicle. Aeroacousticinduced random vibration was calculated using (a) predicted acoustic and aerodynamic noise environments defined in a companion paper; (b) the Condos-Butler scaling method; and (c) reference aeroacoustic and vibration data from Apollo and Saturn V flight and vibroacoustic tests. Random vibration envelopes of $17 \mathrm{~g}-18 \mathrm{~g} \mathrm{rms}$ were derived for five zones of the orbiter fuselage, and $22 \mathrm{~g}$ rms and $81 \mathrm{~g}$ rms for the vertical stabilizer and wings, respectively. Mechanically transmitted random vibration of $22 \mathrm{~g}$ rms was estimated for the aft fuselage from the staged-combustion orbiter engines during orbiter burn, using Saturn S-II flight data as reference and the Barrett scaling relationship. Mechanical shock during boosterorbiter separation was calculated to be an initial peak sawtooth with a maximum of $1.8 \mathrm{~g}$ peak and a duration of 400 
msec. Landing shock was not expected to exceed $1.5 \mathrm{~g}$ peak or $350 \mathrm{msec}$, using a maximum sink speed of $10 \mathrm{ft} / \mathrm{sec}$.

\section{$3: 45$}

GG8. Some Experiments on Fan Noise and Their Implications. MarTin V. Lowson, Loughborough University, England. -Noise output from an open rotor has been measured in an anechoic room. Simultaneously measurements of the aerodynamic input to the fan have been made. The aeroacoustic transfer function has thus been measured and compared to various theoretical models. Results suggest that the fan noise output is due to three sources, distorted inflow, turbulent inflow, and self-generated tip noise. The latter source is significant at higher frequencies and may dominante subjective noise levels for both ventilating fans and low-speed geared fans for aircraft use. It appears that this noise source can be successfully attacked via blade-tip modifications, which can give noise reductions of over $10 \mathrm{~dB}$.

\section{$4: 00$}

GG9. Investigation of Propeller Vortex Noise Including the Effects of Boundary Layer Control. Gerald J. Healy, Lockheed-California Company, Burbank, California 91503.An experimental investigation has been conducted on the vortex noise produced by a two-bladed 4-ft diam model propeller capable of boundary layer removal. The propeller had a spinner comprising $70 \%$ of the total propeller radius. A porous section on both surfaces of the symmetric section airfoil allowed removal of the boundary layer. Free-field measurements were made in an anechoic chamber at three field points for three tip speeds $(209.5,314.2$, and $366.5 \mathrm{ft} / \mathrm{sec})$ and four blade angles $\left(0^{\circ}, 2.5^{\circ}, 5^{\circ}\right.$, and $\left.10^{\circ}\right)$ both without and with boundary layer control. Agreement with theory was good (within $2 \mathrm{~dB}$ ) showing a sixth power of tip velocity relationship and a classical dipole radiation pattern for the over-all SPL of the vortex noise. Boundary layer removal primarily affected sound levels above $3150 \mathrm{~Hz}$, with no ordered effect on the over-all level.

\section{$4: 15$}

GG10. Rotor Noise Attenuation with Leading Edge Devices. Roger E. A. Arndt and Robert T. Nagel, Department of Aerospace Engineering, Pennsylvania State University, University Park, Pennsylvania 16802.-The noise attenuation effects of leading edge serrations on rotors is studied in detail. Total radiated power, farfield SPL, directivity patterns, nearfield sound characteristics, and rotor slip stream and wake characteristics have been compared with rotors with and without leading edge devices. The data collected indicate that a substantial noise reduction is achieved with specific leading edge configurations and running conditions. A strong dependence on rotor speed and bladc pitch angle was found. Spectral analysis of the total radiated power indicates that the attenuation occurs in both the pure tones and the broad-band component of the signal. The data also demonstrate that the broad-band attenuation is shif ted to continuously higher frequencies as the rotor speed increases. Nearfield datal were collected in a manner indicative of rotational noise comcomponents. A considerable noise reduction in the higher harmonics of rotational noise was detected. Thrust and torque data indicate that the addition of leading edge serrations results in a decrease in $L / D$. Hot-wire data show a strong decrease in the near-wake turbulence intensities. It appears that the rotor noise reduction phenomena encompasses both the rotational and vortex components of the noise signal. Reduction in the rotational components is traced to variations in the blade loading. Noise attenuation without performance penalty is therefore realized in the broad-band component only.

$$
4: 30
$$

GG11. Application of External Aerodynamic Diffusion to Reduce Shrouded Propeller Noise. R. E. Longhouse, Center for Acoustical Studies, Department of Mechanical and Aerospace Engineering, North Carolina State University, Raleigh, North Carolina 27607.-A major portion of the noise from V/STOL aircraft fan or shrouded propeller systems occurs during the hover mode. External diffusion of the exhaust flow during this mode significantly increases the thrust/power ratio. Attendant with the flow diffusion is a reduction in fall blade aerodynamic loading. The latter effect suggests a potential source of reducing the fan discrete-frequency noise. Results of model shrouded propeller tests to study the noise reduction associated with external diffusion are presented. [NASA is acknowledged for partial sponsorship of this work.]

$$
4: 45
$$

GG12. Acoustical Modeling of Noise Sources Utilizing Correlation Techniques. B. D. CoOK, Cullen College of Engineering, University of Houston, Houston, Texas 77004.-Acoustical modeling of noise sources is usually performed in the frequency domain. However, there may be advantages in modeling in the time domain using correlation techniques. Examples of the mathematical procedures involved will be illustrated by simple configurations.

\title{
Session HH. Psychological Acoustics II : Frequency and Pattern Discrimination
}

\author{
C. Douglas Creelman, Chairman \\ University of Toronto, Toronto, Canada
}

Contributed Papers (12 minutes)

\section{2:00}

HH1. Intensity and Frequency Discrimination in One- and Two-Interval Paradigms. R. C. Bilger and W. H. JesteadT, Bioacoustics Laboratory, Eye, and Ear Hospital, University of Piltsburgh, Pittsburgh, Pennsylvania 15213. - In an attempt to extend detection theory beyond the intensive domain, both frequency and intensity discrimination data were collected for four subjects using several psychophysical procedures. These included Yes-No, 2 IFC, Same-Different with a positive increment, and Same-Different with a negative incre- 


\section{RD MEETING ACOUSTICAL SOCIETY OF AMERICA}

ment. The two-interval conditions were run with both fixed and random bases. Two increment sizes were used for each condition. The results for intensity discrimination showed the dependencies on method so that the desired intermethod evaluation of frequency discrimination could be effected. Intersubject variability was significantly greater for frequency than for intensity discrimination, but the frequency data for each individual were quite orderly.

\section{2:15}

HH2. Perception of Pitch of Single Period Tones. J. E. Jenkins-LeE, Department of Speech Communication, Case Western Reserve University, and Cleveland Hearing and Speech Center, Cleveland, Ohio 44106, AND E. D. Schubert, Stanford University School of Medicine, Stanford University, Stanford, California 94305. - The ability of the normal human subject to perceive a pitch percept from short-duration pure-tone signals, namely, single period sinuosids centered at 250,500 , 1000 , and $2000 \mathrm{~Hz}$, has been investigated. Instrumentation developed for this research allowed the experiment to be preprogrammed and progress at a rate established by the speed of the subject's trial response. Five subjects completed 20 programmed sequences of 200 presentations. In each 200trial program of the 2IFC paradigm, the reference signal was presented first in exactly 100 of the trials in a predetermined, quasirandom sequence. Five frequency ratios were presented; the values, $\Delta F / F$, were $0.200,0.150,0.100,0.050$, and 0.025 . Psychometric functions derived from the data show that these subjects could reliably differentiate between pitches of single period signals of different frequencies. The mean value of the relative pitch $\mathrm{DL}$ in the $250-$ to $2000-\mathrm{Hz}$ region was found to be $0.10 \pm 0.01$ when the conventional detection criterion, $P(C)$ $=0.75$, was employed. It is noted that the deviation is almost symmetrical about the mean; thus, the value of the averaged relative pitch DL is believed to be approximately constant within the range investigated.

\section{$2: 30$}

HH3. Frequency Discrimination for FM Chirps of Various Bandwidths. Don A. Ronken, Central Institute for the Deaf, St. Louis, Missouri.-Gated sinusoids with linearly changing frequency and essentially rectangular power spectra were used as signals in a frequency discrimination experiment. Signal bandwidths of $10,50,200,500$, and $1000 \mathrm{~Hz}$ were produced by changing the extent of frequency modulation for a fixedduration chirp. The 100-msec FNI chirps were generated digitally with 10 -msec linear rise/fall times and presented at $70 \mathrm{~dB}$ SPL in a continuous wide-band noise of $0-\mathrm{dB}$ spectrum level. One of the two observation intervals in a $2 \mathrm{AFC}$ experiment always contained a chirp that had a center frequency of $750 \mathrm{~Hz}$. The other interval contained an identical chirp, except that its center frequency was $750+\Delta f \mathrm{~Hz}$. The $\Delta f$ required for $75 \%$ correct discrimination $\left(\Delta f_{73}\right)$ was estimated from individual psychometric functions for each of four experienced listeners. As the bandwidth of the chirp increased from 50 to $1000 \mathrm{~Hz}$, the increase in $\Delta f_{75}$ was almost proportional to the square root of the signal bandwidth. FM chirps with a bandwidth of $10 \mathrm{~Hz}$ are slightly more discriminable in frequency than a gated pure tone of the same duration at the same center frequency. The direction of frequency modulation, to higher or lower frequency, had only a small effect, even at the largest bandwidth.

\section{$2: 45$}

HH4. Impairment of Pitch Perception by Temporally Contiguous Stimuli. Dennis H. Holding, Delmar Yoder, and MrChel LOEB, Department of Psychology, University of Louisville, Louisville, Kentucky 40208.-Two experiments were run, combining elements of designs by Elliott (1970) and Massaro (1970), in which noise and pure-tone "masks" bore varying temporal relations to two stimulus tones. The tones, either 20-msec duration ("short") or 400-msec ("long") were initiated 4 sec apart; either the first or second was variable, the other fixed $(1000 \mathrm{~Hz})$. The mask, an 80 -dB white noise or $2193-\mathrm{Hz}_{\mathrm{z}}$ tone $500 \mathrm{msec}$ long was inserted (position 1) 40 msec after the first tone or (position 2) terminated $40 \mathrm{msec}$ before the second tone, or (position 3 ) initiated $40 \mathrm{msec}$ after the second tone. In the first experiment, the variable was 1000 or $1050 \mathrm{~Hz}$ ("coarse discrimination"); in the second, 1000 or $1025 \mathrm{~Hz}$ ("fine"). Discrimination was impaired, especially for short tones, though impairment was minimal in position 2. Impairment was greater by tone than noise in position 1 only and more so for the fine discrimination in position 3 only. One interpretation is that "masking" consists of two kinds of disruption, one associated with storage and one with pitch comparison.

$$
\text { 3:00 }
$$

HH5. Auditory Pattern Discrimination in the Absence of Spectral Cues. BarRY Leshowitz and Raquel Hanzi, Department of Psychology, Arizona Slate University, Tempe, Arizona 85281.--Previous research has shown that brief sounds can be discriminated on the basis of differences in the spectral content of the waveform. The aim of the present work was to assess the importance of spectral processing in the perception of temporal order. In the first study, in an effort to prevent spectral information from serving as the basis for perception of temporal order, sequences of four tones were presented dichotically in a repeated tape loop. Component tones in the sequence were administered to the left or right ear according to a predetermined random order. On each trial, two sequences of tones were presented, and the task of the observer was to determine whether the order of presentation was the same or different for the two sequences. The duration of component sounds required for reliable discrimination was $90 \mathrm{msec}$. The duration threshold for a diotic presentation was 10 msec. A second study investigated the perception of temporal order for high and low tone bursts presented in a nonrepeated sequence consisting of the two tones, a noise, and a buzz. Here the task was to determine whether the order of the high and low tones in the two sequences was the same or different. The location and temporal order of the buzz and noise in the two sequences were randomized and irrelevant to the discrimination. Temporal order could be perceived when the duration of each component sound was 20 msec. [This research was supported by the National Institute of Health.]

\section{$3: 15$}

HH6. Discrimination of the Silent Gap in Two-Tone Sequences of Different Frequencies. PIErre L. Divenyi and IRA J. Hirsh, Central Institute for the Deaf, St. Louis, Missouri 63110.-Pairs of two-tone sequences were presented in such a way that listeners could adjust the time interval separating the onsets of the two tones in the second pair, so that the gap 
or rhythmic repetitiveness appeared to be the same in the second as in the first pair. The pairs consisted of two 20-msec tone bursts with 2.5 -msec rise time and 10 -msec fall time. The time interval separating the onsets of the tones in the first, or standard, pair was $\mathbf{4 0}$ msec. Responses were observed as a function of the frequency of the fourth tone, the standard pair being of one frequency $(1 \mathrm{kHz})$, while the variable pair began at the same frequency but ended with a different one. As the frequency difference increases up to $250 \mathrm{~Hz}$, the time interval in the variable pair for apparent equality of gap decreases, as does the variability in judgments. Beyond a difference of $250 \mathrm{~Hz}$, both quantities increase. These results are consistent with those of earlier time discrimination experiments [P. L. Divenyi, J. Acoust. Soc. Amer. 49, 127 (1971)] and may relate to the time required to shift the listening band from one frequency to another.

\section{$3: 30$}

HH7. Detectability of the Deletion of a Tone from a Tonal Pattern for the Chinchilla. C. K. Bundick, $U . S$. Army Medical Research Laboratory, Fort Knox, Kentucky 40121.-When presented with repetitive, three-tone patterns, chinchillas learned to make an avoidance response to an infrequent change in the frequency of the second tone but did not learn a response to the deletion of the second tone. This, together with other comparable data, may reflect the manner in which the chinchilla uses hearing in its natural environment.

\section{$3: 45$}

HH8. Perception of Temporal Order: Special Rules for the Initial and Terminal Sounds of Sequences. RichaRD $M$. WARren, Department of Psychology, University of WisconsinMilwauke, Milwaukee, Wisconsin 53201.-If a pattern of three or four successive sounds (consisting of hisses, tones, and buzzes) is repeated without pause, listeners cannot identify the order at durations of individual items well above 100 msec, even though each of the component sounds is heard clearly. However, if two of these sounds are each presented once with one following the other, it is possible to identify temporal order for durations below $50 \mathrm{msec}$. It has been suggested that accurate perception of order with such stimulus pairs might involve special mechanisms permitting identification of items at the beginning and end of auditory sequences [Warren, Obusek, Farmer, and Warren, "Auditory Sequence: Confusion of Patterns other than Speech and Music," Science 164,586 (1969)]. The present experiments demonstrate that introduction of a silent interval of $3 \mathrm{sec}$ between repetitions of sequences consisting of three or four successive items each lasting 200 msec facilitates detection of temporal order. It appears that sounds present at initiation and termination of a sequence can be identified readily, and that these cues can be separated experimentally from direct identification of temporal succession. [This research was supported by the National Science Foundation.]

\section{4:00}

HH9. Latency of the Chinchilla's Response to Auditory Change. G. A. Luz, U. S. Army Medical Research Laboratory, Fort Knox, Kentucky 40121.-Chinchillas were tested on auditory pattern discrimination for the set $(A A, B B, A B, B A)$ where $A, B$ stand for two tones and the pattern is 2.25 sec. One of the set was used as a repetitive background signal, which the remaining three were used as signals for instrumental avoidance. Performance was higher when the background was
$A A$ or $B B$ than when it was $B A$ or $A B$. Clear differences between the latency distributions for hits and false alarms were found for the Iormer but not for the latter condition. The data suggest that the chinchilla does not treat a 2.25 sec pattern as a single auditory gestalt.

\section{$4: 15$}

HH10. Proportion of Correct Detections of Rate Changes in Pulsed Stimuli. J. WARM AND Jo ANn DECker, Department of Psychology, University of Cincinnati, Cincinnati, Ohio 45221, AND R. P. SMITH, Department of Psychology, University of Louisville, Louisville, Kentucky 40208.--Two groups of 12 listeners each were required to detect and report-within a 5-sec interval-changes in the rate of ongoing square-wave pulses of 2 and 11 pulses/sec. One group was given preknowledge of the direction of rate change; the other was not. Blank trials, where no change in rate occurred, were inserted randomly into the trial sequences. High proportions of correct detections were associated with short reaction tines and conversely. Preknowledge of the direction of rate change produced no significant effects. The proportion of correct detections was higher for accelerations than for decelerations only at a basal repetition rate of 11 pulses/sec. The percent-correct detection measure closely paralleled the reaction time neasure previously employed by us. Performance efficiency was not affected by the 5 -sec time linitation imposed.

\section{4:30}

HH11. Detecting Signals of Uncertain Frequency: Analysis by Individual Alternative Signals. C. Douglas Creelman, Department of Psychology, University of Toronto, Toronto 181, Ontario, Canada.-Gated sinusoids were presented to highly practiced observers for two-alternative detection. Signal frequencies were randomly chosen from populations that were changed from one run of 100 trials to the next. The populations differed in the range of possible signal frequencies and the number of frequencies that could be chosen from within the range. All feasible combinations of ranges $(50,560,100$, 2000 , and $4000 \mathrm{~Hz}$ ) and numbers of alternatives $(1,3,9$, $17,33,65,129$, and 257 ) were run. When one looks at the observers' performance on signals of specific frequencies, it appears that neither the range nor the number of alternatives alfected performance. Average perforinance differed between ranges because the larger populations encompassed more of the audibility curve.

\section{$4: 45$}

HH12. Binaural Detection in a Sequential Detection Paradigm. J. D. Gilliom, Department of Psychology, University of Alabama, Birmingham, Alabama. 35233.-An extension of the work reported by this author at the Washington meeting, the present study examines sequential detection in two frequency regions assigned to separate earphone channels. The observer's task was to make yes-no decisions concerning the presence of a sinusoidal signal in each earphone channel. A sequential ordering of observation intervals between earphone channels existed for all experimental conditions. The frequency region to be monitored in the right ear was always $630 \mathrm{~Hz}$. Eight experimental conditions were formed by varying whether the order of presentation of observation intervals across earphone chamnels was fixed or random, whether the frequency region assigned to the left ear was at 630 or 1400 $\mathrm{Hz}$, and whether the Gaussian noise background across earphone channels was correlated or uncorrelated. All experi- 
mental conditions were evaluated relative to performance in corresponding single-channel conditions. Results observed include: (1) Knowledge of the presentation order of observation intervals had little effect on performance; (2) A much larger decrement in performance relative to single-channel performance was obtained with a correlated noise background when the frequency region assigned to each earphone channel was the same; no such difference existed when the frequency regions were different. (3) Performance in one channel was lower if a signal event occurred in the opposite channel.

FrIDAY, 21 APRIL 1972

Maple Leaf Room, 9:00 A.m.

\title{
Session II. Noise IV : Community Noise
}

\author{
BASIL BoNk, Chairman
}

\author{
Jackson and Moreland, Boston, Massachusetts 02116
}

\section{Contributed Papers (12 minutes)}

\section{$9: 00$}

IIl. Computer Analysis of City Noise-A Preliminary Algorithm. Richard M. Guernsey, Donley, Miller $\&$ Nowikas, East Hanover, New Jersey 07936.-A preliminary computer algorithm was developed to analyze city noise. The algorithm is eventually to be included in an over-all cost effectiveness program for city noise control. The basic problems, some unsolved in the present state-of-the-art, which have been encountered during the development of this algorithm are discussed. The basic structure of the algorithm is described. It is believed that this algorithm can be readily expanded and improved as the state-of-the-art improves. [This work was supported by Cornell University, Department of Environmental Engineering and Resources for the Future.]

$$
9: 15
$$

II2. Prediction of Road Traffic Noise. U. J. Kurze, Bolt Beranek and Newman Inc., Cambridge, Massachusetts 02138.A computer model for the prediction of noise from freely flowing road traffice is described. The model allows one to account for simple statistics of point sources (spatial distribution and radiated sound power) and for sound propagation with attenuation, diffraction, and reflection. The basic statistical parameters of the Huctuating noise calculated at a receiver are the cumulants of the sound intensity. These cumulants are employed to calculate probability distributions of the SPL as well as single number criteria currently used for the description of fluctuating road traffic noise. The computer model has been developed for the Transportation Systems Center, Cambridge, Massachusetts.

$$
\text { 9:30 }
$$

II3. A Computer-Oriented Highway Noise Ilustrated by the Rhode Island I-84 Environmental Impact Study. LAWRENCE G. Copley, Consultant in Acoustics, Newton, Massachusetts 02158. - A mathematical model of high way noise was developed for use in selecting and evaluating highway corridor locations for minimum adverse noise impact in the adjacent communities. The model is based on the present state of knowledge regarding highway noise, as published by the Highway Research Board and elsewhere. Noise exposure is characterized by $L_{10}$, the 10 -percentile $A$-weighted sound level. The model includes excess attenuation due to atmospheric absorption, topography, and ground cover. The model has been programmed for computer and is used in conjunction with a comprehensive data bank including land use types and terrain information. These data are geographically coded in grid squares of $\frac{1}{5} \mathrm{~km}$ side. Noise impact is quantified by the relation between predicted $L_{10}$ highway noise exposure and what would be completely acceptable for the land use type. The computer output is in the form of two types of shaded maps. One maps the sensitivity to noise impact and can be used in selecting desirable highway corridors. The other type of map displays the noise impact caused by each highway planning alternative.

\section{$9: 45$}

II4. Community Sound Levels: A Comparison of Measured and Estimated Data. H. B. SAFEER, Office of Noise Abatement, Department of Transportation, Il'ashington, D. C. 20590.-The Office of Noise Abatement has developed several models of transportation-generated sound levels. These models are useful in studying the magnitude of transportation noise and forecasting the noise reduction of alternative abatement programs. The Community Median Noise Level Model was used to estimate the median sound levels in Medford, Massachusetts. The estimated median sound levels were compared to the measured median sound levels. A total of 18 sets of measured and estimated average median sound levels were obtained, i.e., eight traffic control zones and the total for Medford, for two time periods. The average absolute difference between the measured and estimated average median sound levels was $1.5 \mathrm{~dB} A$. The Community Median Noise Level Model operates on the following parameters: motor vehicle density, mix by type of vehicle, average vehicle speed and shielding due to structures between the noise source and the receiver. Analysis of the Medford data also confirmed the very strong statistical correlation between the median sound level and the decile sound levels. Regression equations were developed and used to generate estimates of the noise pollution level in Medford, Massachusetts.

\section{0:00}

II5. Community Noise Levels in Medford, Massachusetts. J. E. Wesler, DOT Transportation Systems Center, Cambridge. Massachusetts 02142.-An extensive noise measurement survey was made in Medford, Massachusetts, a suburb of Boston, during March and April 1971, in order to assess the effects of transportation noise in a typical urban community, and to obtain validation data for a mathematical model developed to simulate such noise levels. Noise measurements were made at 49 locations distributed randomly across the community, with continuous $24-h$ measurements at five of these locations. The average median noise levels over the entire community were $55.0 \mathrm{~dB} A$ during the morning traffic 


\title{
83RD MEETING - ACOUSTICAL SOCIETY OF AMERICA
}

rush hours, and $51.8 \mathrm{~dB} A$ during the midday period. Hourly median levels in a typical residential neighborhood varied from a high of $52.9 \mathrm{~dB} A$ from $4: 30$ to $5: 30$ P.M., to a low of 35.1 dB $A$ from 2:30 to 3:30 A.M. Noise levels were influenced primarily by highway traffic, with a major highway arterial corridor producing the highest noise levels in the community. Rail noise from commuter trains and through freights did not significantly affect the community noise levels. Aircraft, overflying Medford from nearby Logan International Airport generated levels in excess of $60 \mathrm{~dB} A$ for roughly $15 \%$ of the measurements period. Repeat measurements at a limited number of locations are planned at semiannual intervals, in an effort to determine general trends in noise levels in this community.

\section{$10: 15$}

II6. Model Study of Aircraft Noise Reverberation in a City Street. L. Pande and R. H. Lyon, Department of Mechanical Engineering, Massachusetts Institute of Technology, Cambridge, Massachuselts 02139.-Experimental studies of sound propagation from a source situated above roof top level in an urban environment have indicated the amplification and shielding effect of buildings. These experiments have been supplemented by diagnostic tests with a spark source which indicate the paths of propagation and their contribution to the received sound. A criterion for reverberation in a city street due to an aircraft is developed in terms of images formed. Charts indicating the amplification or shielding of noise from low flying aircraft are presented.

\section{$10: 30$}

II7. Community Noise Levels of the L-1011 TriStar Jet Transport. NathaN ShaPIRO, Acoustics Staff, LockheedCalifornia Company, Burbank, California 91503.--In order to halt the escalation of aircralt noise in the vicinity of airports, the Federal Aviation Administration developed, and in late 1969 issued, the Noise Certification Standards of FAR Part 36. The Lockheed L-1011 TriStar, a wide-bodied trijet transport, had been planned not only to meet the anticipated FAA requirements, but to provide the quietest community environment that was technologically practicable and economically reasonable. Powered by Rolls-Royce high bypass ratio engines, with quiet operation a major design goal, and utilizing specially designed acoustical lining for its nacelles, the L-1011 has demonstrated fyover noise levels substantially lower than the FAA specified limits, and also measurably lower than those of competitive airplanes. As a result, community annoyance levels have been achieved that are $65 \%$ to $75 \%$ below those of the presently operating older jet transports.

\section{$10: 45$}

II8. Community Noise Survey and Criteria for a Power Station-A Case Study. C. M. Salter AND R. M. Hoover, Bolt Beranek and Newman Inc., Cambridge, Massachusetts 02138, aND II. E. BradLEy, Stone and W'ebster Corporation, Boston, Massachusetts 02107.-Power companies are expecting to double their electrical generating facilities every 10 years, and in order to build new facilities these companies need the approval of local and state agencies. In this particular study, the power company had a $560-\mathrm{MW}^{\prime}$ facility completed in the late 1960's and petitioned the state for permission to build an additional $560-\mathrm{MW}$ facility. The acoustical criterion proposed by engineers for Unit 2, now being designed, was nondegradation of the present ambient. The sound level of the combined plant must not exceed the present ambient level of 46-50 dB $A$ at the nearest neighbors in all directions. To achieve this criterion, noise sources will be quieted both in the existing unit and in the future unit. This paper presents the results of our initial community ambient noise survey around the existing plant made to establish the present conditions. The individual noise sources determined at that time to be distinct contributors to the plant noise in the community are described. These sources include a $400-\mathrm{MW}$ autotransformer, air intake louvers to forced draft fans, the main 560-.11W transformer, and boiler feed pump equipment. SPL data of these sources, both close-in and in the community, are presented as examples of some typical community noise problems associated with power plants. Also the requirements for noise control as related to the planned addition are discussed.

\section{1:00}

II9. Predicting Community Response to Blast Noise. P. D. Schomer, U. S. Army Corps of Engineers, Construction Engineering Research Laboratory, Champaign, Illinois 61820.A preliminary method is given to predict annoyance levels in the environs of a blasting area. The means are given to scale $X$ pounds of TNT to $1 \mathrm{lb}$ of TNT; buried charges and above ground detonations are also considered. Various ways to predict probable blast overpressure and spectrum as a function of distance on days exhibiting adverse weather conditions are considered. The annoyance of man by blast noise is predicted using the composite noise rating (CNR) and, for this calculation, the impulsive noise startle correction factor suggested by Kryter is added to the perceived noise level. Blast noise case histories are considered for verification of the prediction method, and the prediction method is suitable for computer computation of "equal annoyance" contours.

FRIDAY, 21 APRIL 1972

EMBASSY ROOM, 9:00 A.M.

Session JJ. Psychological Acoustics III : Contributed Papers

\author{
William Melnick, Chairman \\ Ohio State University, Columbus, Ohio 43210
}

\section{Contributed Papers (12 minutes)}

$9: 00$

JJ1. Attenuation in Custom-Fitted Earplugs. JERRY V. ToBias, Civil Aeromedical Institute, Oklahoma City, Oklahoma 73125.It seems obvious that personalized or custom-fitted earplugs should provide a better seal within the ear canal than should standard, over-the-counter earplugs. Tests of more than a dozen varieties of insert hearing protectors demonstrated that this reasoning leads to the wrong conclusion. The best earplugs tested were among the least expensive. Even waximpregnated fiber is better (for most people) than most per- 
sonalized earplugs. A few exceptions have been noted, and some discussion of them is provided, as is an attempt to specify the possible sources of ineffectiveness in specially made plugs.

\section{$9: 15$}

J]2. Masked Threshold at Reduced Ambient Pressure. Howard N. McGregor and Albert A. GalipeaU, Martin Marietta Corporation, Denver, Colorado 80201.-Differences in signal tone perception between $1 \mathrm{~atm}(14.7 \mathrm{psia})$ and $\frac{1}{3} \mathrm{~atm}$ (5.0 psia) in the presence of background noise were investigated. In addition to the generation of a background noise spectrum conforming to that specified for spacecraft of the Skylab program (NC 55 approximately), predicted acoustic properties of the spacecraft were simulated. SPLs of three signal tones were experimentally varied using the "Block Up-and-Down, Two-Interval Forced-Choice" technique. Masked thresholds were determined for the three signal tones under the two air pressure conditions and compared with predicted masked thresholds and subjective preferences. The data indicate that the difference in ambient pressure between $\mathbf{1 4 . 7}$ and 5.0 psia does not significantly affect masked thresholds, nor does this difference in ambient pressure affect subjective preferences for signal tone SPLs or sound quality. A further finding indicated that reduced pressure significantly affected the SPL of sound producing equipment in that the SPL decreased with a decrease in pressure but not in accordance with the expected formula $10 \log _{10}\left(\rho c / \rho_{0} \mathcal{C}_{0}\right)$. [This study was supported by a NASA contract.]

\section{9:30}

JJ3. Diver Auditory Sensitivity: Another Look at Bone Conduction. S. H. Feinstein, H. Hollien, and P. Hollien, Communication Sciences Laboratory. University of Florida, Gainesville, Florida 32601.--Support for the hypothesis that divers hear primarily by bone conduction was obtained by Hollien and Brandt [J. Acoust. Soc. Amer. 46, 384-387 (1969)]. In a further test of that hypothesis, the thresholds of seven submerged listeners were obtained (at frequencies of $0.25,0.50,1.0,4.0$, and $8.0 \mathrm{kHz}$ ) under three different conditions: (1) Wearing a full $\frac{3}{16}$-in. wet suit with no hood; (2) wearing a full $\frac{3}{16}-$ in. wet suit with a $\frac{3}{16}-$ in. hood; and (3) wearing a full $\frac{3}{16-i n}$. wet suit and hood with $\frac{1}{4}$-in. rubber tubes passing through the hood to the meatuses. There were no significant differences between conditions two and three but thresholds were significantly lower in condition one. This finding provides further support for the hypothesis that underwater sound energy is transduced by bone conduction rather than by the normal middle-ear linkage.

\section{$9: 45$}

JJ4. Auditory Sensitivity of the Owl Monkey. M. D. BEECHER, New England Regional Primate Research Center, Harvard Medical School, Southborough, Massachusetts 01772.-Puretone absolute thresholds for the owl monkey (Aotus trivirgatus) were determined using a tracking procedure. The animal was trained to lick a dry spout; occasional licks produced a tone, following which a lever response (detection) produced food reinforcement. No-tone trials were included to allow an estimate of the animal's false-alarm rate, which for all animals was less than $5 \%$. Threshold testing was conducted in a sound-treated chamber, and tones were presented from a high-fidelity or electrostatic loudpseaker. On threshold sessions, the SPL was decreased $3 \mathrm{~dB}$ after each correct detection and increased $3 \mathrm{~dB}$ following each miss. The owl monkey audibility curve is characterized by best sensitivity at 8-10 $\mathrm{kHz}$ and $+40 \mathrm{~dB}$ SPL cutoffs at approximately $125 \mathrm{~Hz}$ and $42 \mathrm{kHz}$. The high-frequency slope is very sharp, the $+70 \mathrm{~dB}$ SPL cutoff occurring at approximately $45 \mathrm{kHz}$. The audibility curve of this nocturnal monkey is similar to those of other monkeys which have been tested. [Supported by the National Science Foundation and by the National Institute of Neurological Diseases and Stroke.]

\section{0:00}

JJ5. Loudness Discomfort Levels for Pure Tones and Noise. D. E. Morgan, R. H. Wilson, and D. D. Dirks, Department of Head and Neck Surgery (Audiology), UCLA Medical School, Los Angeles, California 90024.-Two studies have been conducted to measure the loudness discomfort levels (LDLs) for subjects with normal hearing. First, the LDL for a $1000-\mathrm{Hz}$ stimulus was measured utilizing the method of constant stimuli, the method of adjustment, and a tracking method. The method of constants provided the most reliable judgments among subjects over six trials. In the subsequent study, LDLs were measured utilizing the method of constants for pure tones at octave frequencies from 125 through $4000 \mathrm{~Hz}$ and for two bands of random noise. LDLs (in $\mathrm{dB}$ SPL) varied as a function of the stimulus employed. LDLs were highest at low frequencies (through $500 \mathrm{~Hz}$ ) and relatively constant for 1000,2000 , and $4000 \mathrm{~Hz}$ and for the wide-band noise.

\section{$10: 15$}

JJ6. Binaural Analysis as a Function of Physiological Masking. J. W. Lindsey AND D. R. Soderquist, Department of Psycholagy, University of North Carolina, Greensboro, North Carolina 27412.-Five normal-hearing subjects served as observers in a yes/no signal detection task for four different frequencies $(100,200,300$, and $5000 \mathrm{~Hz})$. Two binaural listening conditions were used at all frequencies such that the interaural phase was either $0^{\circ}(\mathrm{S} 0)$ or $180^{\circ}(\mathrm{S} \pi)$. Signals had a duration of $100 \mathrm{msec}$ and a rise-decay time of $10 \mathrm{msec}$. All data were collected at absolute threshold. Binaural sensitivity $\left(d^{\prime}\right)$ was examined as a function of two signal onset delays in terms of the cardiac cycle (EKG $R$ wave) of each subject. The selected delays were either coincident with the $R$ wave $(0.0 \mathrm{sec})$ or $0.5 \mathrm{sec}$ later. The experiment was an attempt to investigate the possibility that the absolute threshold varies as a function of physiological masking provided by cardiac sounds. Initial results indicate substantial detection advantages associated with the 0.5 -sec time delay. These data as well as the decrease in this advantage as frequency increased suggest that signals presented coincident with the EKG R wave are masked by the cardiac sound.

\section{0:30}

JJ7. Temporal Relations in Low-Frequency Pure-Tone Central Masking. Bradley L. Billincs aNd T. E. STokinger, Veterans Adminislration Hospital and University of Oklahoma Medical Center (Department of Communication Disorders), Oklahoma City, Oklahoma 73104.-Low-frequency contralateral masking was investigated with pulsed (500-msec) $200-\mathrm{Hz}$ pure-tone maskers presented at $10,30,50$, and 70 dB SL. Brief pure-tone maskees ranging in frequency from 170 to $4000 \mathrm{~Hz}$ were located near the onset or at the midpoint of the maskers. Four normal-hearing subjects participated in this experiment; a transformed up-and-down psychophysical 
method was utilized for the determination of unmasked and masked thresholds. Some existing experimental evidence of an indirect nature suggests that overshoot may be minimal for low-frequency contralateral maskers. The results of the present study, however, indicate that overshoot for low-frequency contralateral maskers appears greater than that which has been determined by other investigators at higher masker frequencies. Selected ipsilateral masking conditions were also investigated. These results will be discussed in relation to the contralateral masking data and to other ipsilateral masking studies.

\section{$10: 45$}

JJ8. Changes in the Relative Magnitude of Auditory Adaptation with Additional Measurement Conditions. ERNEst M. Weiler, Harry Blackmond, and Karen Hippard, Speech Department, University of Cincinnati, Cincinnati, Ohio 45221.For $10 \mathrm{Ss}$, more adaptation was measured when midplane localization was used before and alter asymptotic exposure than when instructions requested midplane localization prior to exposure and loudness balances following, as previously reported by Stokinger and Studebaker (1968). However, when Ss were requested to make a loudness balance before adaplation and the midplane localization aflervards, the greatest amount of adaptation was measured. Analysis of variance revealed significantly more adaptation for conditions requesting preadaplation loudness balances than preadaptation midplane localization. Conversely, significantly more adaptation was measured for postasymptotic measurements requesting midplane localization than for loudness balances. Conclusions suggest the value of testing complete combinations of variables, if possible. A suggestion that learning may influence midplane localization versus loudness balance comparisons seems reinforced by two additional listeners who were self-trained musicians. They reported a $500-\mathrm{Hz}$ tone in one ear and 4000 $\mathrm{Hz}$ in the other as a single dissonant chord, localized according to relative intensity of the tones. Milner's (1970) discussion of time-order versus intensity difference localization suggests relationships with learning phenomena.

\section{1:00}

JJ9. Interaural Intensity Relations associated with Median Plane. Steven W. Vargo and Raymond Carhart, Auditory Research Laboratories, Northwestern University, Evanston, Illinois 60201 . - The interaural intensity relations associated with median plane and left- and right-ear lateralization thresholds were studied in a group of 10 normal-hearing young adults using a method of adjustment. Two tonal stimuli-250 and $2000 \mathrm{~Hz}$-were presented in a counter-balanced order at an SPL of $60 \mathrm{~dB}$ to the fixed ear. Subjects were instructed to adjust the intensity to the variable ear in order to (1) position the intracranial sound image at the midsaggital plane following a 30-sec period during which the subject crossed and recrossed his judged median plane; and (2) position the intracranial sound image at the location where he judged the stimulus to be just perceived in the appropriate ear starting from the median plane. Results will be reported with respect to the normal range in intensity present for the lateralization positions as well as intensive magnitude (in decibels) of the ear-bias effect reported in previous studies of lateralization phenomena.

\section{1:15}

JJ10. A Redetermination of Binaural Equal-Loudness Contours for Normal Hearing Observers. James T. YATES,
Hearing Clinic, Texas Tech University, Lubbock, Texas 79409, Robert M. Johnson, Speech and Hearing Clinic, University of Denver, Denver, Colorado 80202, and William J. STarz, General Rose Hospilal, Denver, Colorado 80220.-The relationship of perceived loudness across frequency was investigated with $\mathbf{4 0}$ adult subjects. A method of contant stimuli was employed whereby the subjects made equal-loudness judgments to Tones of $125,250,500 \mathrm{~Hz}$, and $2,3,4,6$, and $8 \mathrm{kHz}$ compared to a $1 \mathrm{kHz}$ reference. Equal-loudness contours were established at 10-dB intervals from 18 to $108 \mathrm{~dB}$ SPL. An especially constructed apparatus generated, programmed, and regulated the stimuli and produced reference and comparison pure tones with less than $1 \%$ harmonic distortion at all frequencies tested. Some important differences between the present results and those of previous authors were noted. First, the equal-loudness contours were of less severe slope at the lower frequencies than those of previous investigators. Second, the characteristic notch at $8 \mathrm{kHz}$ shown by previous studies was seen, but was shifted to $6 \mathrm{kHz}$.

\section{$11: 30$}

JJ11. Acoustical Wavetrain Signals and Equal Loudness Pressures. John A. Victorisen, Victoreen Laboratory, Maitland, Florida.--Equal loudness measurements were made for 12 young adult ears over a frequency range of $250-6000 \mathrm{~Hz}$. A damped wavetrain signal having a decrement of 0.90 was used and this was delivered by a pair of circumaural earphones. A well-defined but empirical relationship is shown to exist between relative loudness and sound pressure and the mathematical expression is given. Observational accuracy attainable was, when averaged, usually consistent with the calculated values to within about $\frac{1}{2} \mathrm{~dB}$ over a pressure range of about 0.0001-100 $\mu$ bars. With this method of measurement comfortable louduess pressure for the normal ear was found to be independent of frequency over the normal frequency range. Damped wavetrains with circumaural earphones appeared to give about four times greater accuracy than pure tones and supraaural earphones for identical measurements.

\section{$11: 45$}

JJ12. Human Monaural Localization of Noise. R. W. GateHOUSE AND W. Cox, Department of Psychology, University of Guelph, Guelph, Ontario.-Eight normals matched on age and sex with eight monaurally deaf subjects were compared on their ability to localize a brief white noise source. The monaurals were classified as sensorineural, and "severely" to "profoundly" deaf (ISO, 1964). The subjects' task was to localize eight $45^{\circ}\left(0^{\circ}\right.$ azimuth reference) positions on each of five planes (the aural axis and planes $15^{\circ}$ and $30^{\circ}$ above and below it). Four different predetermined blocks of 40 randomized trials each were used. Therefore, each subject localized each position four times for a total of 160 responses per subject. The trial blocks were presented in two different orders to half of the subjects in each group. Matched subject pairs received the same order. The results showed that (1) monaural localization was above the chance level on all horizontal planes, although significantly inferior to binaural localization; (2) in the vertical planes, binaural and monaural localization ability did not differ; (3) monaurally, "normal" ear-side localization is highly accurate and the mean size of errors on that side is smaller; (4) for both groups errors were made by displacement of sounds to the rear of the "true" position: (5) head movements are not necessary for accurate monaural localization. These results will be discussed in the light of several previous findings. 


\title{
Session KK. Experiments with Sound in the Sea
}

\author{
Raymond IV. HASse, Chairman \\ Naval Linderwater Systems Center, New London, Connecticut 06320
}

Contributed Papers (12 minutes)

9:00

\begin{abstract}
KK1. Spatial Coherence of Sound Propagated in Deep-Water and Shallow-Water Geometries. J. C. MoLdon, Defence Research Establishment Atlantic, Darimouth, Nova Scolia, Canada. - Scattering of sound from the sea surface and sea bottom causes losses in spatial coherence of the propagated sound field. Ocean measurements have been made of the wavefront coherence in both vertical and horizontal directions at frequencies of $2.4,4.7$, and $9.4 \mathrm{kHz}$ in moderate sea-state conditions. Omnidirectional source and receivers were used, and results are presented for two geometries: (1) deep water in which only a single surface reflection is encountered and (2) shallow water where multiple surface and bottom reflections exist. Variations in coherence with source and receiver depths are described, and results are compared with theoretical estimates of the angular dispersion produced by scattering at the rough boundaries.
\end{abstract}

$$
\text { 9:15 }
$$

KK2. Acoustic Measurements in the West Greenland Sea. D. L. Bradley AND G. M. ColviN, Nazal Ordnance Laboratory, Silver Spring, Maryland 20910.-Acoustic ambient noise, transmission loss, and reverberation measurements were made in the West Greenland Sea at the edge of the permanent polar ice pack during the fall of 1971 . The data were collected with the assistance of the U. S. Coast Gaurd, while deployed on one of its oceanographic data collection cruises. The sound sources for transmission loss and reverberation measurements were standard U. S. Navy explosive sound signals. The receiving system consisted of calibrated sonobuoys that were monitored both from helicopters and the Coast Guard icebreaker. Ancillary environmental data were provided by the Coast Guard and ice conditions and ice cover information was provided by U. S. Navy ice observers. The results of data analysis are presented and compared with data from similar arctic environmental conditions. [Work supported by U. S. Naval Air Systems Command.]

$$
\text { 9:30 }
$$

KK3. Convergence Zone Propagation in the North American Basin. John D. Shaffer, Naval Research Laboratory, Washington, D. C. 20390.-The long range propagation of a CW 14$\mathrm{Hz}$ signal was studied experimentally. A shallow source was towed across the North American Basin to a maximum range of about $2700 \mathrm{~km}$. The signal was received on a sound channel hydrophone and the envelope recorded. Well-defined convergence zones were observed over the major extent of the tow. The existence of this mode interference wavelength as a sharp spectral line is shown in a power spectrum calculation of the envelope squared versus range.

\section{$9: 45$}

KK4. Sound Transmission from Deep to Shallow Water. R. J. URICK, Naval Ordnance Laboratory, Silver Spring, Maryland 20910.-The propagation of sound from deep to shallow water was studied by a direct field experiment. Two aircraft were used : one to drop explosive sound signals detonating at 800 and $2000 \mathrm{ft}$ out to 100 miles in two directions off the coast of Delaware, the other to monitor a pair of sonobuoys located in $100 \mathrm{f}$ of water at the break of the continental shelf and at a point 20 miles toward shore in shallow water. The field-recorded data were converted to transmission loss by standard techniques. Surprisingly good transmission was observed. The 100-f receiver showed lower transmission losses for the deep axial shots than it would have if it had been located on the channel axis in deep water. This good trans mission is possibly due to two causes : a kind of focusing effect, wherein the continental slope acts to increase the density of rays at its top, plus a near-optimum slope condition enabling axially transmitted sound to take but a single bounce up into shallow water. The 800 -ft shots were also well-received in spite of the absence of all-water ray paths, probably as a result of conversion to seismic waves along the slope.

\section{0:00}

KK5. Noise Signature of an Aircraft in Level Flight over a Hydrophone in the Sea. R. J. URICK, Naral Ordnance Laboratory, Silver Spring, Maryland 20910.--An aircraft fying over a hydrophone in the sea produces a noise signature whose magnitude and duration depend on such parameters as hydrophone depth, water depth, and light altitude. This signature represents sound reaching the hydrophone in four different ways: via a direct refracted path, via one or more botton reflections, via the so-called lateral wave, and via sea-surface scattering. Of these, the refracted path is the most important and easiest to evaluate. Generalized contours for the nearfield of this component are given; in the farfield it is shown that the source in air can be replaced by an equivalent in-water source having a cosine-squared directivity pattern and a source level $7 \mathrm{~dB}$ less than that of the real source. In a field experiment, a Navy P3 Orion turboprop aircraft Hew over hydrophones at two depths in deep watcr. The brief noise signature, lasting but a few scconds, was found to be accounted for satisfactorily by the refracted sound field. However, under conditions not existing in the ficld experiment, such as in shallow water, in rough seas, or at very shallow depths, it is conceivable that the other contributors to the total underwater sound field may become important.

\section{0:15}

KK6. Lloyd Mirror Effects in Sounds from Aircraft. B. Harris, Ocean \& Almospheric Science, Inc., Dobbs Ferry, New York 10522, S. Torak and G. Lavge, U. S. Naval Air Development Center, Jahnstille, I'arminster, Pennsylania 18974. - In the altomatic monitoring of environmental noise, it would be useful to use vertical directivity as an aid in discriminating between noise from overflying aircraft and noise from vehicles. However, the benefits of utilizing directivity can be mitigated by the Lloyd mirror effect in which the aircraft noise not only propagates as a direct ray but as ground-reflected ray as well. The purpose of this experiment was to measure the Lloyd mirror signals from actual aircraft moving at reasonable speeds. These signals were broad-band 
recorded using omnidirectional microphones. Since the Lloyd mirror effect is more apparent in narrow-band signals as consecutive reinforcements and cancellations between the direct and reflected ray, the signals were narrow-band filtered using the fast Fourier transform algorithm. Signal processing was then used to remove the statistical variations of the aircraft noise as well as the variations of the local ambient noise. The final results show amplitude variations of the order of 10 $\mathrm{dB}$ at the times predicted by the theoretical models. This means that utilization of sound directivity requires microphones with nulls both at the top and bottom of the microphone pattern.

\section{0:30}

KK7. BIFI-Normal-Mode Generation with a Vertical Array of Projectors. L. A. King, New London Laboratory, Naval Underwater Systems Center, New London, Connecticut 06320.This paper describes first results from a continuing study of normal-mode generation and propagation in shallow water. A novel experimental tool used in this study is a vertical array of 25 sound projectors. With it, fundamental work has been conducted using its directional properties to transmit acoustic energy in an optimal manner. The basis of this work presumes the shallow-water channel to propagate "favored" inodes. Their optimal transmission should occur by steering the acoustic energy with the array into boundary-grazing angles associated with the development of these modes. Ground work was laid using the array without time delays or amplitude shading to study patterns generated under a wide variety of propagation conditions. Measurements, made in March 1971, are presented here that depict patterns observed under isothermal conditions.

\section{$10: 45$}

KK8. Sound-Speed Dispersion and Fluctuations in Coastal Ocean Waters. Juergen Rautmann and Herman Medwin, Physics Department, Naval Poslgraduate School, Monterey, California 93940.-In-situ upper ocean measurements have revealed the existence of significant dependence of the speed of sound on frequency over the range $25-80 \mathrm{kHz}$. At $4.3-\mathrm{m}$ depth the variation of speed with frequency went through a large resonancelike peak of $+6 \mathrm{~m} / \mathrm{sec}$ and dip of $-3 \mathrm{~m} / \mathrm{sec}$ relative to the predictions for bubble-free water. The behavior is attributed to near surface resonant bubbles with an identifiable population around radius $55 \mu$ at $4.3 \mathrm{~m}$ below the surface and a lesser population centered around $125 \mu$ with numbers decreasing with depth to $14.3 \mathrm{~m}$ (in 16-m-deep water). The temporal fluctuations of the speed were principally at surface wave orbital frequencies and showed nearGaussian probability density functions with rms values from 0.27 to $0.52 \mathrm{~m} / \mathrm{sec}$. The Gaussian character is disturbed and the rms value is greater at the dispersion center frequencies. [Supported by Naval Ship Systems Command.]

\section{1:00}

KK9. Pressure Dependence of High-Frequency Sound Attenuation in the Deep Pacific Ocean. H. F. BEzDEK, University of California, San Diego, Marine Physical Laboratory of the Scripps Institution of Oceanography, San Diego, California 92152.- Vertical and horizontal acoustic paths, located in the Pacific Ocean between depths of 910 and $3350 \mathrm{~m}$ have been utilized to determine the attenuation of sound at a nominal frequency of $75 \mathrm{kHz}$. Results from these two different geometries show that predicted values exceed the observed magnitudes of acoustic attenuation at this frequency and at these depths. At $3350 \mathrm{~m}$, the predicted value is $22.7 \mathrm{~dB} / \mathrm{km}$ vs a measured value of $13.3 \pm 0.5 \mathrm{~dB} / \mathrm{km}$, a discrepancy of more than $9 \mathrm{~dB}$. Furthermore, increasing pressure reduces the at- tenuation, a fact which supports the work of Fisher [J. Acoust. Soc. Amer. 38, 805 (1965)] in contrast to that of Kester and Pytkowicz [Geochimica et Cosmochimica Acta 34, 1039 (1970) ]. However, the amount of reduction is larger than previously suspected by more than $80 \%$. [This paper represents results of researcl sponsored by the Office of Naval Research.]

\section{$11: 15$}

KK10. Project Hiawatha Revisited: Application of FFP to Lake Superior Attenuation Experiment. D. G. Browning, M. R. Powers, R. H. Mlellen, and F. R. DiNapoli, New London Laboratory, Naval Underwater Systems Center, New London, Connecticut 06320 .- Owing to the pressure dependence of the temperature of maximum density in fresh water, the sound channel in Lake Superior is extremely weak. The fast field program (FFP) is applied to determine if the bottom loss is significant for sound channel propagation in the kilohertz region. The values of attenuation are computed for the frequency range $500-10000 \mathrm{~Hz}$ and compared to values obtained from the Lake Tanganyika experiment.

\section{$11: 30$}

KK11. Seamount Reflections from the CANNIKIN Underground Explosion. JohN Northrop, Naval Undersea Research and Development Center, San Diego, California 92132.--Hydroacoustic signals were recorded from the five megaton Amchitka Island underground explosion (code name CANNIKIN) of 6 November 1971. SOFAR depth hydrophones of the Missile Impact Location System (MILS) off the islands of Midway, Wake, and Oahu in the Central Pacific were used as detectors. In the low-frequency $(<20 \mathrm{~Hz})$ band, the main signal was followed by reverberations that persisted for several hours after the shot. Arrival times of some of these reverberations were correlated with those computed for reflections from seamounts in the Emperor Scamount Chain and the Hawaiian Arch. Reverberations from more distant reflectors were separated by quiet periods or "windows" of several minutes duration. The azimuthal and temporal distribution of the "windows" versus the reverberation maxima is investigated in relation to low-frequency ambient noise in the ocean.

\section{1:45}

KK12. A Mathematical Model for Volume Reverberation. I. Experiment. IV. J. Jonst, Naval Ordnance Systems Command, Department of the Navy, Ilashinglon, D. C. 20360.-An experiment was conducted to measure parameters for a Poisson model for volume scattering. Using a stable platform suspended beneath a ship, echoes from discrete scatterers were observed and analyzed. A cumulative probability distribution of scatterer reflectivity (ratio of scattered to incident pressure) was obtained by examining more than 1200 individual echoes. The average number of scatterers per unit volume was estimated from experimental data. The envelopes of echoes from scatterers were similar to the envelope of the transmitted signal.

\section{2:00}

KK13. A Mathematical Model for Volume Reverberation. II. Simulation. IV. J. JoBsT, Nazal Ordnance Systems Command, Department of the Navy, Washington, D. C. 20360, AND T. I. SmITs, Department of Electrical Engineering, Catholic University of America, IVashington, D. C. 20017.-A technique for simulating reverberation due to small fish or other discrete scatterers is presented. A simulated ocean volume is filled with Poisson distributed scatterers according to a model proposed by $D$. Middleton. Each scatterer is assigned a random location and a random reflectivity. By adjusting the simula- 


\title{
83RD MEETING ACOUSTICAL SOCIETY OF AMERICA
}

tion parameters, the simulated reverberation envelope was made to agree statistically with the envelope of reverberation observed experimentally in the ocean. The simulation is particularly useful for estimating the true probability distribution of scatterer reflectivity and true scatterer density from experimental data influenced by a transducer beam pattern.

FrIDAY, 21 APRIL 1972

Georgian Room, 9:00 A.M.

Session LL. Speech Communication VI : Temporal Aspects of Perception

Arthur House, Chairman

Institute of Defense Analyses, 100 Prospect Avenue, Princeton, New Jersey 08450

\begin{abstract}
At this session preprints will be distributed for contributed papers. There will be no formal presentations; discussion only.
\end{abstract}

\section{Contributed Papers}

LL1. Effects of Interruption on Noncontextual Speech. N. T. Hopkinson, R. C. BILger, AND M. D. WANG, School of Medicine, University of Piltsburgh, Pittsburgh, Pennsyluania 15213.- The distortion of speech by interruption and its effects on intelligibility were studied in six listeners with normal hearing. Rates of interruption were varied between 2 and $250 \mathrm{ips}$, and duty cycles were $50 \%, 25 \%$, and $12.5 \%$. The signals were a closed message set of $16 \mathrm{CV}$-nonsense syllables that had been recorded on a drum. The portion of the CV. syllable presented was varied systematically, that is onset of the syllable was delayed parametrically. Random presentation of signals was accomplished by use of a computerized system. In conditions of $50 \% \mathrm{D}$. C., at the lowest rate of interruption, transmitted information was highest when there was no delay in onset of interruptions; however, transmitted information dropped significantly when the delay reached a critical time with respect to the period of time the signal was on. At the highest rate of interruption, transmitted information remained high in all conditions of delay in onset of interruption. Duty cycles less than $50 \%$ showed lower amounts of transmitted information. Feature analyses in terms of transmitted information will be reported for the various conditions.

LL2. "Unbiased" Procedure for Comparing Degree of Lateralization of Dichotically Presented Stimuli. RICrard HarshMaN, Phonetics Laboratory, UCLA, Los Angeles, California 90024, and Stephen KRAshen, Southwest Regional Labaratories and UCLA, Los Angeles, California.--In some experimental designs, it is desirable to compare "degree of lateralization" for different stimuli or different groups. Current measures of ear difference make such comparisons difficult, because scores are influenced by subjects' relative accuracy in identifying the stimuli. One reason for this "bias" is demonstrated using some simple mathematical models of a subjects' behavior in dichotic listening. A scoring method is deduced that is not biased by different amounts of guessing by subjects. This procedure, the percent of errors method (POE), consists of computing each ear's percentage of the total number of errors. The utility of the POE method is confirmed by reanalysis of previously published data. Standard methods for measuring ear difference are found to be negatively correlated with changes in accuracy. POE scores, however, do not correlate significantly with accuracy scores. This is true whether accuracy differences are due to age, first versus second channel reported, or differences in stimulus difficulty. Reanalysis of consonants versus vowels, younger versus older subjects, and other comparisons of degree of lateralization suggest some alternatives to previous conclusions.
LL3. Lateralization and the Critical Period. Stephen KRASHEN, Southwest Regional Laboratories, 11300 La Cienega Blud., Inglewood California 90304 and UCLA, Los Angeles, California, and Richard Harshman, Phonelics Laboralory, UCLA, Los Angeles, California 90024.-The percent of errors method, described in Harshman and Krashen above, was applied to dichotic listening data for children between 5 and 10 years old. No significant change in degree of lateralization was found. This supports the hypothesis that the development of lateralization is complete by age five, rather than by puberty, as argued by Lenneberg. Data cited by Lenneberg, as well as additional cases, were examined. Analysis of cases of acquired aphasia in children resulting from localized lesions revealed that right-sided lesions do not cause more cases of speech disturbance after five than in adults, indicating that the involvement of the right hemisphere in the language function in children older than five is the same as in adults; this also supports the age five hypothesis. Not enough crucial data is available from hemispherectomies to decide between the two hypotheses. The completion of lateralization by five implies that lateralization reflects the maturation of some essential component of the language faculty, rather than the termination of "organizational plasticity," as suggested by Lenneberg.

LL4. Interaural Intensity Differences in Dichotic Speech Perception. C. L. Thompson, M. Stafford, J. K. Clllen, JR., L. Hughes, S. Lowe-Bell, and C. I. Berlin, Kresge Hearing Research Laboratory, L. S. U. Medical School, New Orleans, Louisiana 70119.- Three studies are reported in which the intensities of dichotic stimuli were varied. In Study 1, one signal remained at $80 \mathrm{~dB}$ SPL. while the other was varied from 30 to $80 \mathrm{~dB}$ in $10-\mathrm{dB}$ steps. In Study 2, one ear was held at $50 \mathrm{~dB}$ while the other ear's signal was varied from 30 to $80 \mathrm{~dB}$ in 5 -dB steps. In Study 3, the signals were varied from 30 to $80 \mathrm{~dB}$ SPL in $10-\mathrm{dB}$ steps but with equal intensities to the two ears. Eleven subjects were used in each of the former studies and 12 in the latter. Study 1 shows asymmetry in lavor of the right-ear messages even when they are $10 \mathrm{~dB}$ less intense than the left. Data of Studies 2 and 3 also show asymmetries but in a more complex fashion.

LL5. Temporal Processing of Speech. Patricia KuHL and Charles SPEAKs, Speech Research Laboratory, University of Minnesola, Minneapolis, Minnesola 55455.-Differences between intelligibility of interrupted and compressed speech were examined by holding speech information constant and varying duration of silent intervals between speech segments. We hypothesized that interrupted speech would be more intelligible than compressed speech because silent intervals could 
be used to "process" the fragmented speech segments. Sentences and words were sampled by computer with a $50 \%$ duty cycle. Two discard intervals, 125 and $50 \mathrm{msec}$, were used. Sample segments were retrieved and arranged with varying silent intervals between segments ( 3 less than, 1 equal to, and 2 greater than the duration of original discard intervals), producing six experimental conditions. When sample segments for the sentences were abutted with no silent interval (compression), intelligibility deteriorated. When silent intervals were inserted, intelligibility improved regardless of whether the silent interval was less than, equal to (interruption), or greater than the original discard interval. Since intelligibility did not vary significantly with the amount of "processing" time inserted, perhaps the silent interval simply "marked" the place where speech had been removed. Neither the "processing time" or "marking" hypotheses explain the results for word intelligibility.

LL6. Perception of Voice Timing in Spanish Stop Consonants. Arthur S. Agramson," and Leigh Lisker, † Haskins Laboratories, New Haven, Connecticut 06510.-In earlier acoustic analysis of Spanish, we showed that speakers differentiate voiced / bdg/ from voiceless /ptk/ in word-initial position by means of voice onset time (VOT), the temporal relation between the onset of glottal pulsing and acoustic features of supraglottal articulation. More recently, we demonstrated the perceptual efficacy of VOT for Thai, a language with three categories along the timing dimension, and English, a language with two categories. The two Spanish categories differ from those of English in their placement of the phonetic boundary. Spanish voiced stops are produced with VOT values that lead the consonant release, while voiceless stops show VOT upon release or immediately thereafter. In the present study, native speakers of Spanish identified randomized recordings of stops synthesized with VOT varying in small steps. Labeling responses showed a fairly good fit between production and perception differing from English in the expected direction. The listeners also discriminated the VOT variants in a psychoacoustic test format. The latter results, along with the earlier ones for English and Thai, indicate that discriminability is primarily determined by specific language experience, although general phonetic categories may have some effect.

- Also at University of Connecticut, Storrs, Connecticut 06268.

† Also at University of Pennsylvania, Philadelphia, Pennsylvania 19104.

LL7. How Competing CVs, Vowels, or Synthetic Bleats Affect Dichotic Scores of Temporal Lobectomees, Hemispherectomees, and Normals. C. I. BerLin, S. Lowe-Bell, R. PorTER, H. BerLin, and C. L. Thompson, Kresge Hearing Research Laboratory, L. S. U. Medical Center, New Orleans, Louisiana 70119. - Three normals, three hemispherectomees, and three temporal lobectomees were stimulated dichotically with CVs in the contralateral ear (left ear for normals) always at $75 \mathrm{~dB}$ SPL; the ipsilateral ear (right ear for normals) received either a different $\mathrm{CV}$, a "bleat," or an envelopeshaped vowel. The ipsilateral ear was stimulated at intensities ranging in $10-\mathrm{dB}$ steps from SRT to $75 \mathrm{~dB}$ SPL. Data show that an ipsilateral "bleat" had suppressive capabilities even with normals, despite little intelligibility of its own. All ipsilateral stimuli suppressed the patients' contralateral ears drastically compared to the normals even if the ipsilateral stimuli were $20-30 \mathrm{~dB}$ less intense than the contralateral CVs. The amount of contralateral $\mathrm{CV}$ intelligibility loss caused by a simultaneous signal in the ipsilateral ear may be a sign of the latter's "speechlike" qualities.

LL8. Extension of Interaural Delay for Intelligibility. S. E. Gerber, Speech and Hearing Center, University of California, Santa Barbara, Ca. 93106.-At the previous meeting we presented some data to suggest that a notch appears in the intelligibility function at integral multiples of $50 \mathrm{msec}$. The data were extended only to delays of 150 msec on a relatively small group of listeners. The present paper reports data on a virtually identical study extended now to 275 msec in 25 msec steps, with and without noise added, and tested on a highly reliable listening panel. The significance of these data is discussed, and consideration is again given to the coherence of the signal and the noise.

LL9. Mutual Interference between Two Linguistic Dimensions of the Sarne Stimuli. Ruth S. DAy, Haskins Laboratories and Yale University, New Haven, Connecticut 06510, and Charles C. Wood, Neuropsychology Laborafory, Veterans Administration Hospital, West Haven, Connectiut 06516. - In a previous study subjects identified binaural stimuli that varied along both a linguistic and a nonlinguistic dimension. The linguistic dimension consisted of variation in stop consonants while the nonlinguistic dimension consisted of variation in fundamental frequency. There were four stimuli: /ba/-low, /ba/ high, /da/-low, /da/-high. Reaction times were obtained in a two-choice identification task when the target dimension was the only one that varied. When there was also irrelevant variation in the nontarget dimension, reaction times increased substantially for the linguistic dimension, but only slightly for the nonlinguistic dimension. Thus the nonlinguistic dimension interfered with the processing of the linguistic dimension more than vice versa. The present study employed the same paradigm, but used two linguistic dimensions: stop consonants and vowcls. The stimuli were /ba, bx, da, da/. Reaction times increased substantially for both dimensions when there was also irrelevant variation in the nontarget dimension. Thus both dimensions interfered with each other to the same extent. On the basis of the dimensions examined in this paradigm thus far, it appears that two linguistic dimensions yield a mutual interference effect, while a linguistic and a nonlinguistic dimension yield a unidirectional effect.

LL10. Dichotic Fusion along an Acoustic Continuum. James E. Cutring and Ruth S. Day, Haskins Laboratories and Department of Psychology, Yale University, New Haven, Connecticut 06510.-When stimuli such as banket and lanket are presented dichotically, phonemic fusions often occur: subjects report hearing blanket. Previous studies have shown that stop $+/ r /$ and stop $+/ l /$ items have different fusion properties. For example, /1/ was sometimes substituted for $/ r /$ (but rarely vice versa) : gocery/rocery $\rightarrow$ (yielded) glocery. The present experiment varied the liquid stimuli along an acoustic continuum involving the third formant transition. For example, one set varied from ray to lay. Each was paired dichotically with an initial stop stimulus, in this case pay. All inputs (pay, ray, lay) and possible fusions (pray, play) were acceptible English words. When asked to report "what they heard," subjects gave many fusion responses. Of these, there was a preponderance of stop $+/ l /$ fusions ( $88 \%$ vs $12 \%$ ). They occurred even for pairs where the liquid item was reported as an $/ r /$ during separate binaural identification trials. Thus, given that an item was identified as ray, the same subjects reported hearing play when it was paired with pay: pay/ray $\rightarrow$ play. Despite the fact that the third formant transition is crucial for perception of $/ r /$ vs $/ 1 /$, this parameter was not responsible for the observed phoneme substitutions.

LL11. The Effect of a Word Boundary on the Perception of Timing in Speech. A. W. F. HuGGins, Research Laboratory of Electronics, Massachusetls Institule of Technology. Cambridge. Massachusetts 02139.-Earlier work has shown that a change in the duration of one acoustic segment in a sentence sometimes does, and sometimes does not, require a compensatory change in an adjacent segment [A. W. F. Huggins, J. Acoust. Soc. Amer. (to be published)]. For example, in the words for paupers, compensation was required between the vowel in for and the initial $p$ in paupers, but was not required between the 
initial $p$ and the stressed first vowel in paupers. These and other results suggest that the presence or position of a word boundary may influence the need for compensation. An experiment has been designed to investigate more carefully the role played by a word boundary. The results have implications for the organization of speech production.

LL12. Temporally Segmented Speech. A. W'. F. HugGins, Research Laboratory of Electronics, Massachusetts Institute of Technology, Cambridge, Massachusetts 02139.-Alternated and interrupted speech both show a minimum of intelligibility when the bursts of speech reaching the listener last about 150 msec. A similar effect occurs with "temporally segmented" speech, which is made by splicing silent intervals into a recording of continuous speech, thus dividing it up into "speech intervals" separated from each other by "silent intervals."
The advantage of this material is that the durations of speech and silent intervals can be independently varied, without discarding any of the speech. An earlier experiment [see MIT Res. Lab. Electron., Quart. Progr. Rep., No. 103 (1971), p. 126.] suggested that the dip in intelligibility is actually due to two separate effects: (1) the probability that a speech interval will be recognized decreases as its duration decreases and (2) the probability that signal parameters can be followed from one speech interval to the next decreases as the duration of the intervening silent interval increases. Experimental support for this view has been obtained using temporally segmented speech (1) by varying the duration of speech intervals with silent intervals held constant and (2) by varying the duration of silient intervals, with speech intervals held constant. [This research was supported by $\mathrm{NIH}$ Grant No. NSO4332.]

FrIDAY, 21 APrIL 1972

Buffalo Room, 9:00 A.M.

\title{
Session MM. Physical Acoustics II : Propagation of Ultrasound in Various Media
}

\author{
B. D. Cook, Chairman
}

University of Houston, Cullen College of Engineering, Houston, Texas

\section{Contributed Papers (12 minutes)}

\section{9:00}

MM1. The Measurement of the Bulk Modulus Loss Factor of Small Solid Specimens. Geoffrey L. WrLsov, The Pennsyluania Slale Universily, University Park, Pennsylvania 16802. - The bulk modulus loss factor of small, relatively lossfree solid specimens is one of the most elusive elastic constants to measure, as it is essential that the losses in the apparatus be reduced below those of the sample. One technique uses the change in decay time when the sample is introduced into a resonant body of liquid. Early experiments involved the use of a longitudinal standing wave in a tube, but losses due to wall friction were excessive. Wall friction losses have been minimized by the use of a radial resonance in a spherical container, and with the use of a vacuum jacket to reduce acoustic radiation, a $Q$ as high as 80000 has been achieved; however, there were practical difficulties due to asymmetries in the vessel and to multiple resonances in the walls. The present experiments involve a compromise, using a predominantly radial mode in a cylindrical vessel. Both thin walled (resilient) and thick-walled (nearly rigid) vessels have been used. The limitations inherent in these measurements will be discussed.

\section{$9: 15$}

MM2. Multistate Dissociation and Sound Absorption in Sea Water. F. H. Frsher and H. F. Beznek, University of California, San Diego, Marine Physical Laboratory of the Scripps Institution of Oceanography, San Diego, California 92152.-At $75 \mathrm{kHz}$, the observed decrease of sea water sound absorption with increasing pressure is about twice that predicted from the multistep dissociation parameters selected by Eigen and Tamm [Z. Elektrochem. 66, 92-121 (1962)] to explain sound absorption in aqueous solutions of $\mathrm{MgSO}_{4}$, the salt responsible for sound absorption in sea water. To account for such a large pressure effect requires the equivalent of a single chemical reaction with a $\Delta V \simeq 36 \mathrm{~cm}^{3} /$ mole; for example, two successive chemical reactions each with a $\Delta V \simeq 18 \mathrm{~cm}^{3} / \mathrm{mole}$. The results are discussed in terms of three-step and two-step dissociation models. Included in our considerations are Millero's results [Geochimica et Cosmochimica Acta 35, 1089-1098 (1970)] obtained independently of acoustic data, which show only a small decrease with pressure for the concentration of $\mathrm{MgSO}_{4}$ ion pairs. [This paper represents results of research sponsored by the Office of Naval Research and the National Science Foundation.]

$$
9: 30
$$

MM3. Relaxational Ultrasonic Absorption in Molten Selenium. E. M. Ring* AND R. T. BEYER, Brown Universily, Physics Department, Providence, Rhode Island 02912.-The temperature and frequency dependence of the ultrasonic attenuation in molten selenium have been studied in the range from $200^{\circ}$ to $400^{\circ} \mathrm{C}$, and from $30 \mathrm{MHz}$ to $70 \mathrm{MHz}$. The data are consistent with the literature in the high temperature range from $350^{\circ}$ to $600^{\circ} \mathrm{C}$, but supersede previous results in the range from $200^{\circ}$ to $350^{\circ} \mathrm{C}$. Analysis of the data is complicated by the structure of molten selenium, which is thought to possess a double structure of rings and chains, in equilibrium at any temperature. The material has been treated as a polymer in the literature, and by comparing the observed relaxations in Se with those reported in other polymers the present data can be analyzed: the monomer-polymer structure gives rise to corresponding nonrelaxing and relaxing components, of which the monomer (rings) represents a nonrelaxing solvent, and the polymer (chains) a relaxing solute. Finally, once the frequency dependence of relaxations occurring at constant temperature has been mapped into the temperature dependence of relaxations occurring at constant frequency, the previously reported high-temperature data can be fitted by a single relaxation time model. [Supported by the O. N. R.]

* Present address: Industrial Research Products, Inc., 321 N. Bond St., Elk Grove Village, III. 60007.

\section{$9: 45$}

MM4. Reflection of an Elastic Wave from a Free Surface in Quartz. Gerald L. Jones and Edmund G. Hennrke II, Department of Engineering Mechanics, Virginia Polylechnic Institule and State University, Blacksburg, Virignia 24061.The reflection of a plane, elastic wave in quartz is discussed. Numerical solution has been obtained for quasilongitudinal and quasitransverse waves incident upon the $x y, x z$, and $y z$ crystal planes for angles less than critical. The angles of the reflected wave vectors, the associated reflection coefficients, and the directions of the energy flux vectors for the reflected 
waves have been calculated. The numerical results indicate that an incident critical angle occurs when a reflected wave has a Poynting vector parallel to the boundary as suggested in an earlier paper [E. G. Henneke II, J. Acoust. Soc. Amer. $51,210(1972)]$. In some incidents, the wave vector of the reflected wave may be pointing in a direction in free space, but if its Poynting vector is directed internally, the reflection coefficient is nonzero. In addition, it has been found that for some incident angles greater than critical, three quasitransverse waves may be reflected, and the boundary conditions are satisfied without the necessity of assuming a surface wave. [This work was supported by the National Science Foundation].

\section{0:00}

MM5. Brillouin and Rayleigh Scattering Studies of Electrolyte Solutions. Arthur Maret and Ernest Yeager, Case Western Reserve University, Cleveland, Ohio 44106.-Rayleigh and Brillouin scattering studies have been carried out in a number of electrolyte solutions at various concentrations up to $4 M$. The apparatus consisted of an argon laser operating in a single mode, a thermostated octagonal light scattering cell with provisions for scattering at precisely known angles, a piezoelectric scanning Fabry-Perot interferometer, and a photomultiplier-photon-counting system. Three types of results have been obtained: (1) hypersonic velocity data from the frequency shift of the Brillonin components; (2) hypersonic absorption coefficients from the line widths of the Brillouin components; and (3) Rayleigh-Brillouin intensity ratios. The first and second types of results have been found to compare very favorably with those from ultrasonic velocity and absorption data at lower frequencies. The theoretical Rayleigh-Brillouin intensity ratios have been calculated from concentration fluctuation theory and found to be in good agreement with experiment at concentrations up to several molal except for 2-2 electrolytes such as $\mathrm{MnSO}_{4}$ and $\mathrm{MgSO}_{4}$. [Research supported by the Office of Naval Research and a NATO grant.]

\section{$10: 15$}

MM6. Speed of Sound in Liquid Metals and Alloys. JACEK JARZYNSKI, Naval Research Laboratory, Washington, D. C.
20390.- The speed of sound $c$ in liquid metals and alloys is calculated using the perturbation theory of liquids and the pseudopotential theory of metals. An elfective hard-sphere diameter, as defined by the Gibbs-Bogoliubov inequality, and its density dependence are determined for each metal from thermodynamic data. The structure independent contribution to $c$ is calculated from the expression for cohesive energy given by Heine and IIeaire [Solid State Phys. 24 (1970)]. The results, including the temperature and pressure dependence of $c$, are in quantitative agreement with experimental data.

$$
10: 30
$$

MM7. Macromolecular Interaction in the Absorption of Sound in Erythrocytes. F. IV. Kremkau, E. L. Cartensen, and W. G. AldRIDGE, Departments of Electrical Engineering, Radiation Biology (Biophysics), and Anatomy, University of Rochester, Rochester, New Fork 14627.- Whereas it has been recognized for some time that the absorption of sound in suspensions of erythrocytes is primarily a macromolecular phenomenon, recent observations have led us to believe that interactions between macromolecules may play a significant role as well. Two tests of the interaction postulate are reported: (1) Bovine erythrocytes fixed in acrolein and glutaraldehyde are sufficiently stable that they can be subjected to fairly harsh environmental changes without loss of hemoglobin or change in microscopic structure. This stability results from cross-linking of macromolecular hydrophobic groups by the fixing agent. The specific absorption of fixed erythrocytes is greater than that for normal cells by as much as a factor of 3.5 at $90 \mathrm{MHz}$. The new relaxing elements added by fixing are concentrated at frequencies above $10 \mathrm{MHz}$. (2) Interaction of ionic groups should be increased by decreasing the dielectric constant of the suspending medium. Suspension of fixed erythrocytes in the solvents ethylene glycol and ethanol further increases the absorption by as much as a factor of $\mathbf{3 . 5}$ at $10 \mathrm{MHz}$. The relaxing elements added with solvent change are skewed toward frequencies below $30 \mathrm{MHz}$. Scattering, viscous relative motion, and thermal absorption have been ruled out as explanations for the observations. The explanation is more apt to lie in some forms of chemical or structural relaxations.

FridAy, 21 April 1972

Empire axd Washixgton Rooms, 9:00 a.m.

\section{Session NN. Engineering Acoustics III}

\section{E. J. Parssinen, Chairman}

Naval Underwater Systems Center, New London, Connecticut 06320

\section{Contributed Papers (12 minutes)}

9:00

NN1. Four-Pole Parameters for Hyperbolic Horns. Charles T. Molloy, TRW Syslems Group, MeLean, Virginia 22101.-It is the purpose of this paper to define, outline the derivation of, and show some applications of the four-pole parameters of hyperbolic horns. These horns were first discussed by $\mathrm{V}$. Salmon [J. Acoust. Soc. Amer. 17, 199, 212 (1946)] and include as special cases exponential, catenoidal, conical, and cylindrical horns. Their four-pole equations are a pair of linear equations relating the pressure and volume velocity existing at the throat to the same quantities at the horn mouth. The coefficients in the equations are called the four-pole parameters. The equations may be written in matrix format and are very convenient for analyzing acoustical systems containing hyperbolic horns as elements (as well as combinations of many other acoustical elements). The four-pole parameters for the general hyperbolic horn and its important special cases will be given. Examples of their uses will be given including: throat impedance; insertion loss; horn-type sidebranch filters; horns with arbitrary area functions; loud speaker systems; etc. It is pointed out that, because the fourpole parameter technique involves only $2 \times 2$ matrices, it is particularly well adapted for use with high-speed digital computers.

NN2. Conical and Sinusoidal Horns as Sound Traps. B. N. Nagarkar, H. C. Dhingra, and R. D. Finch, Deparlment of Mechanical Engineering, University of Houston, Housion, 
Texas 77004.-It is shown that the application of Webster's theory to sinusoidal and conical horns leads to the conclusion that such horns, used as receivers and terminating in cusps, should act as sound traps. This result is further discussed in light of three-dimensional conical horn theory. Experimental work to verify the theory is in progress.

NN3. Transmission-Line Analogs For Stacked Crystals with Piezoelectric Excitation. A. Barlato, U.S. Army Electronics Technology \&o Devices Laboratory, U. S. Army Electronics Command, Fort Monmouth, New Jersey 07703, H. L. BERTONI AND T. TAMIR, Department of Electrical Engineering and Electrophysics, Polytechnic Institute of Brooklyn, New York 11201. -A piezoelectrically driven thickness-mode structure containing any number of stacked crystal layers is shown to be rigorously representable by equivalent circuits, which consist of transmission lines and lumped elements. Each crystal stratum is homogeneous but may be arbitrarily anisotropic; the piezoelectric excitation may be applied to a single plate or several plates in combination, its field orientation being either normal or parallel to the layers. The equivalent representation is derived by considering a canonic two-layered element, which is thereafter utilized as a building block for the analysis of general multilayered stacks. In such a configuration, the material in each layer is accounted for by at most three acoustic transmission lines; each layer boundary, on the other hand, is represented by lumped transformer elements that serve to interconnect all of the six transmission lines arriving at that particular boundary. The piezoelectric drive appears as an additional simple network which is also connected to the coupling transformers. This establishes a procedure for a systematic treatment of theoretical and practical problems concerning crystal resonators and filters, delay lines, pulse and code generators, and related applications.

\section{$9: 45$}

NN4. Effect of Initial Stress on the Propagation of Flexural Waves in Elastic Rectangular Bars. J. A. ClARK, A. J. DuRELLI, AND P. A. LAURA, Electrical Engineering Department, The Catholic University of America, Washington, D. C. 20017. - The dynamics of continuous media subjected to initial stress is of great interest in several fields of applied science and technology: geophysics, oceanography, underwater acoustics, and structural design. Pioneering analytical work in this field was done by Biot. Few contributions have been made, however, to the experimental treatment of the problems of determining dynamic stress distributions within statically prestressed continuous media. The present paper deals with an experimental analysis of the propagation of Hexural waves in prismatic elastic bars with and without prestressing. A complete, direct, full-field optical determination of dynamic stress distributions associated with the flexural waves is obtained by a combination of photoelastic and interferometric measurements. The effects of prestressing by axial tension, axial compression, and pure bending are illustrated. Comparisons are made with an approximate theory. The results are extended to bars of other materials by scaling laws.

10:00

NN5. "Clackers" Noise. D. G. Holmes and R. H. Lyon, Department of Mechanical Engineering, Massachusetts Institule of Technology, Cambridge, Massachusetts 02139.-Impact sounds are normally the result of acoustic radiation from the vibrations of the impacting bodies. The energy pathway is from the preimpact kinetic energy of translation, via the energy of vibration, to the radiated acoustic energy. An alternative mechanism exists, which does not require vibrations of the impacting bodies. The sound radiation is a result of the sudden accelerations of the bodies during impact, and can be said to draw on the kinetic energy, not of the bodies themselves, but of the added mass of the fuid motion. The sound radiated from the impact of two small, hard spheres is a good example. Experiments are described which identify clearly the sound source as the sudden accelerations due to impact; vibrations of the spheres make a negligible contribution to the total energy radiated. The energy spectrum and directivity of the radiation agree very closely with calculated values.

\section{0:15}

NN6. Transient Response Analysis and Experiment on a Transducer with an Intermediate Layer of a Quarter Wavelength for the Generation of Short Uitrasonic Pulses. YosHImitsu Kikushi, Daitaro OkuYaya, and Chihiro Kasai, Research Institule of Electrical Communication, Tohoku University, Sendai 980 , Japan.-In the field of ultrasonic flaw detection, ultrasonic diagnosis, etc., generation and detection of short ultrasonic pulses are very important for high range resolution. In this paper, the transient characteristics of the transducer with an intermediate layer of a quarter wavelength is analyzed by an impulse response method, and the optimum acoustic impedance of the layer for the generation and detection of the shortest ultrasonic pulses is given as a function of specific impedances of the transducer and an acoustic load. The features of this analysis are that the calculation is much simpler than that in the usual frequency response method, and especially that the pulse length which is the final object of the art can be directly derived. From the calculation, it is found that the transducer with the layer of the optimum impedance can generate fairly short ultrasonic pulses without any acoustic backing, and that consequently the transducer sensitivity is considerably higher than that of the usual transducers with backing. Experiments were carried on in megahertz range with $\mathrm{PZT}$ disks using Bakelite or tungsten-resin mixture for the intermediate layer. The results showed fairly good agreement with the theory.

10:30

NN7. A New Broad-Band Ultrasonic Technique with Biomedical Implications. I. Background and Theoretical Discussion. Joie Pierce Jones and Hugh A. Wright, Bolt Beranek and Newman Inc., 50 Moulton Street, Cambridge, Massachusetts 02138 .- Ultrasonic techniques presently used for medical diagnosis make use of narrow-band or single-frequency pulseecho devices. Recent studies at $B B N$ indicate that a new broad-band technique should significantly improve present diagnostic capabilities. This new technique makes use of acoustic impulses, which are short in time but broad in frequency content, and a time domain deconvolution procedure. The result is a temporal waveform which can be related to physical parameters such as specific acoustic impedance and frequency-dependent attenuation. Thus, the new technique allows us to measure quantitatively to a high degree of accuracy the specific acoustic impedance at an arbitrary position within a test object.

\section{$10: 45$}

NN8. A New Broad-Band Ultrasonic Technique with Biomedical Implications. II. Preliminary Experiments Involving Human Skull Bone. Joie Pierce Jones and Hugh A. Wright, Boll Beraneh and Newman Inc., 50 Moulton Street, Cambridge, Massachusetts 02138. - A broad-band acoustic pulse, produced by a spark source operating in water, was used to illuminate several test objects. The reflected signal was analyzed using a deconvolution procedure and the specific acoustic impedance obtained as a function of time. In one particular experiment, 
a series of modest impedance differences were accurately resolved behind a section of human skull bone.

\section{1:00}

NN9. Acoustical Pattem Recognition of Knee Joint Diseases. M. L. Chu and I. A. Gradisar, Department of Mechanical Engineering, The University of Akron, Akron, Ohio 44325.-An improved sound retrieval system and more accurate analysis technique were developed to extract distinguishing acoustical signatures of sound emitted by normal and pathological knee joints during active movements. Background noise was minimized using the principle of "noise cancellation" with a double microphone-differential amplifier setup. Two identical sensitive condenser microphones and an FM recorder with flat responses in the audio range were used. The knee noise was found to be transient in nature and recordings were made on magnetic tape "loops" for "real time" analysis. Waveform, spectral, and statistical (correlation and probability) analyses were performed. Preliminary studies of 30 knee recordings covering normal, rheumatoid arthritic, degenerative knees, and knees with Reiter's syndrome showed that their respective waveforms, spectral patterns, and statistical properties are, on the average, unique and proved to be a promising nondestructive diagnostic tool for early detection of the type and extent of knee joint damage.

\section{$11: 15$}

NN10. Reflection of Acoustic Waves from a Stably Stratified Atmosphere. F. F. Hall, Jk., and W. D. NefF, Wave Propagation Laboratory, NOAA Environmental Research Laboratories, Boulder, Colorado 80302.-Echoes obtained with a monostatic acoustic sounder have to date been attributed largely to random thermal structure in the atmosphere. A dependence of echo strength on the sound beam zenith angle has now been verified for certain highly stable conditions in the planetary boundary layer. Maximum returns are obtained with a vertically pointed beam. The regions of strong return correlate spatially with temperature striations which are essentially horizontal. Measured microthermal structure of such inversion layers can account for the excess or Fresnel acoustic reflection observed. This excess echo may be several times stronger than scattering from random thermal structure. Examples of simultaneous returns at zenith angles of $0^{\circ}$ and $45^{\circ}$ are presented, and the theory of normal acoustic reflection from realistic temperature inversions discussed.

$$
11: 30
$$

NN11. Small-Size High-Efficiency Underwater Loudspeaker. Alan H. Lubell, Lubell Laboratories Inc., Columbus, Ohio 43209. - An improved underwater loudspeaker covering the frequency range from $200 \mathrm{~Hz}$ to $5 \mathrm{kHz}$ has been developed. This transducer combines small size and high efficiency. It yields speech reproduction comparable to medium fidelity air acoustic loudspeakers. This device has been used successfully as part of a portable underwater communication system for instruction and recall of a group of swimmers or divers. Using the underwater hearing thresholds of Brandt and Hollien, estimates of range are made for the above system and compared to test results.

$$
11: 45
$$

NN12. Gradient Microphone Performance within a Gas Mask. Charles T. Morrow, Advanced Technology Center, Inc., Dallas, Texas 75222.--It has been shown previously that a pressure gradient microphone is so insensitive to standing wave effects in a diving mask that it provides excellent intelligibility, but it does not compensate for detuning of the first formant. Detuning by a gas mask is a more major effect and more complicated-the first two formants are split into a triplet. The uppermost resonance of the triplet, corresponding closely in frequency to the original second formant, is attenuated. The gradient microphone, close to the lips, restores intelligibility by deemphasizing the middle resonance of the triplet and restoring emphasis on the uppermost.

FrIDAY, 21 APRIL 1972

Maple Leaf Room, $2: 00$ P.M.

\title{
Session OO. Noise V : Noise Measurement and the Effects of Noise on Man
}

\author{
John J. Earshen, Chairman \\ Cornell Aeronautical Laboratory Inc. and State University \\ of New York at Buffalo, Buffalo, New York
}

Contributed Papers (12 minutes)

2:00

OO1. Individual Differences in the Annoyance of Noise. O. L. Angevine, JR., Anderson and Angevine, Inc., East Aurora, New York 14052.--Among the factors influencing individual appraisals of noise intrusion or annoyance are the information contained in the noise, the person's conditioning, and his interpretation of what the noise represents. His previous adaptation to the noise, and his expectations as to the level and the difficulty of quieting it also affect his estimate. Cultural differences in conversational voice level and speaking distance affect the evaluation of background noise. Daily and monthly rhythms occur in sensitivity. All of these factors serve as a caution that the individual response may depart from the "average" reaction.
$2: 15$

002. Relation of Hearing Impairment to Noise Exposure and Age. James H. Botsforn, Bethlehem Steel Corporation, Bethlehem, Pennsylvania 18016.-An equation relating prevalence of impaired hearing to age and noise exposure has been derived. It is based on the assumption that the probability of developing impaired hearing at any age is proportional to the fraction of the population of that age which has already developed impaired hearing. In other words, the stress on the auditory system of living to any age is indicated by the prevalence of impaired hearing at that age. If there is also exposure to dangerous noise, the probability of impairment increases with the severity of the exposure. The solution of the differential equation resulting from these assumptions 
reveals a complex relationship of age and noise exposure to impairment. The ellects of age and noise are shown not to be simply additive as is of ten assumed.

\section{2:30}

003. In-Ear Measurement of Noise. A. J. Brammer AND J. E. PIercy, National Research Council of Canada, Division of Physics, Ottawa, Ontario, Canada.-A measure of the sound field at the base of the concha, obtained by using a miniature microphone located within the ear, provides information on the acoustical signal entering the ear canal. When combined with a portable tape recorder, the apparatus can be used to evaluate an individual's exposure to noise and appears to be particularly suited for use in directional sound fields that vary in the vicinity of the head. In order to compare the data obtained with existing criteria for hearing conservation, or sound level meter measurements in a diffuse field, a transfer function is used to produce the equivalent diffuse field that would exist at the center of the head in the absence of the subject. The device has been used to measure the noise levels experienced by operators of snowmobiles and chain saws as a function of time, and the attenuation and, in some cases, amplification of sound produced by some common Iorms of crash helmet and headgear. An over-all accuracy of $\pm 2 \mathrm{~dB}$ is obtained from 100 to $5000 \mathrm{~Hz}$ by using a spectrum shaper and a built-in calibration system.

\section{$2: 45$}

004. Auditory Temporary Threshold Shift in Snowmobile Users. R. B. Chaney, JR., and S. C. McClain, Department of Speech Pathology and Audiology, University of Montana, Missoula, Montana 59801.-It has been determined that the noise levels of many snowmobiles and other all-terrainvehicles frequently exceed criterion values of safe exposure as a function of time. However, there are obvious limitations to the use of the CHABA criteria for determining hazardous noise exposure, since typical snowmobile use does not approximate the extent of exposure implied by the "workinglifetime" concept. Thus, the empirical determination of the amount of TTS resulting from "typical" exposures, whatever they may be, seemed to provide a more relevant approach to the question of whether snowmobile noise poses a hazard to hearing acuity of operators and passengers. TTS measurements were obtained on a number of individuals of all ages under field conditions (within 1-15 min of termination of exposure). Significant amounts of TTS were observed to be occurring in a large proportion of the persons tested. The implications of this study are discussed in terms of the users, the manufacturers, and the CHABA criteria for hazardous noise exposure.

\section{3:00}

OO5. Noise Levels on Aircraft Carrier Flight Decks and Their Effects on Humans. J. C. WEBSTER, Human Faclors Technology Division, Naval Electronics Laboratory Center, San Diego, California 02152.--Measurements were made of noise levels produced by four aircraft during pilot qualification exercises aboard the flight deck of USS KITTY HAWK. These measurements, on both the $A$ - and $C$-weighting networks, were augmented by calculations of speech-interference levels made hater from tape recordings. These data were compared to similar measurements made at the Naval Air Test Center at Pautuxent River, Maryland, and interpreted in terms of deafness risk and interference with speech communications. The levels measured on the carrier showed large amounts of low-frequency energy (at octaves centered at 62 and $125 \mathrm{~Hz}$ ) not present in the data taken ashore; this variation is ascribed to the presence of blast deflectors on the carrier and to the effects of strong wind across the deck and the measuring microphone in its wind screen. The noise levels measured are shown to severely degrade speech communications and to present a risk of deafness to personnel. Measure the noise levels produced by aircraft landing on and taking off from flight decks of aircraft carriers. Assess these levels in terms of (1) the hearing hazards they present to personnel exposed to them for various periods of time, with varying degrees of ear protection, and (2) the extent to which they degrade speech communication. When noise exposures were extrapolated to a 12-h cyclic air operation, hearing hazards were predicted, even when current ear-protection devices were utilized. Direct observation together with calculation of speech-interference levels indicated significant decrement in voice communication under high-noise conditions.

\section{$3: 15$}

006. Noise and Measurement Problems Associated with Hard-Hat Diving Helmets. A. L. DiMatria, CBS Laboratories, Stamford, Connecticul 06905, and J. J. Pennella, Naval EOD Facility, Indian Head, Maryland 20640.-The deep-sea hard-hat diver must be supplied with a copious air flow through his helmet at rates far in excess of his need for life oxygen. If reckoned in terms of free air movement, this flow increases with depth of dive and is required to reduce carbon dioxide content resulting from exhaled diver's breath. At a typical depth, the flow rate may be $28 \mathrm{ft}^{\mathrm{a}} / \mathrm{min}$ or about 800 liters/min. All this air passing through a number of control valves, entrance ports, and discharge valves causes much turbulence, with attendant noise known to reach well into the damage risk levels. This paper explores some special problems encountered in the measurement of diver helmet noise and describes a series of noise measurements performed in a typical helmet under simulated diving conditions.

\section{$3: 30$}

007. A Wearable Pocket Noise Dosimeter. M. W. BAsch, General Radio Company, Concord, Massachusetls 01742.-A noise dosimeter that meets the ANSI Type II Sound-Level Meter standard and accumulates the Walsh-Healey percentage is discussed. The dosimeter is composed of two partsa small pocket unit that accumulates and stores the digital data representing the Walsh-Healey percentage and an indicator unit that provides a digital readout on a light emitting diode display. The indicator unit also has provision for calibrating the pocket unit (including the microphone) and provision for checking batteries. Newest technology has permitted the pocket unit to be made very small and light. It includes a small ceramic microphone, a true rms detector with more than 15-dB crest factor capacity, and an extremely low-power MOS digital counter to store the Walsh-Healey percentage. It weighs but $7 \mathrm{oz}$ and runs for over $300 \mathrm{~h}$ on an ordinary 9-V "transistor" battery. Frequency response measurements will show the effects of the proximity of the wearer.

\section{$3: 45$}

O08. Education by Noise Control Projects. F. G. HaAG, Department of Mechanical Engineering, Lnion College, Schenechady, N. Y. 12308.- Voise control presents many interesting possibilities for student-project-type courses. The projects provide a natural motivation at a low cost to educational benefit ratio. Projects have been successfully conducted with undergraduates in general education, and with undergraduate and graduate engineering students. Among the more recent projects are effect of snowmobile noise on mice, standing wave tube, visible sound apparatus, computer simulation programs, muffler design, wave-tank analogy, noise reduction 
demonstrator, noise cancellation device, noise reduction of a computer card reader, and the effect of noise on plants. Since most noise control projects involve the student in value judgments, an auxiliary educational benefit results.

\section{4:00}

009. Design and Operation of a Continuous, Areawide Aircraft-Noise Monitoring System. Gordon Bricken, Northrop Corporation, Anaheim, California, AND John K. Hilliard,
Ramberg \& Lowrey, Santa Ana, California.-A five-station, permanent, 24-h centralized aircraft noise monitoring system has been installed in the community surrounding Orange County Airport, Santa Ana, California. This paper will discuss the design concept, the results obtained from initial tests involving measurement of over 1000 Hights, the practical experience gained from the installation, the true value to the community, and the potential for elfective noise management programs. In addition, the measurement data will be used to demonstrate the value of the Calilornia noise index CNEL.

FRIDAY, 21 APRIL 1972

Georgian Room, $2: 00$ P.M.

\title{
Session PP. Speech Communication VII : Analysis, Identification, and Recognition of Speech and Speakers
}

\author{
JoHN F. Bоенм, Chairman \\ National Security Agency, Ft. George G. Meade, Maryland 20755
}

\section{Contributed Papers}

\begin{abstract}
PP1. Implementation of an On-Line Speaker Verification Scheme. R. C. Lummis, Bell Telephone Laboratories, Murray Hill, New Jersey 07974. - The implementation is described of an on-line version of the scheme for speaker verification reported previously. A Honeywell DDP-516 computer is used, with microphone and keyboard input, disk storage, and graphic output. Utterances are converted to pitch and gain contours and compared with similar functions fetched from disk that represent past vocal behavior of the speaker whose identity is claimed. The comparison process includes automatic temporal registration. Special features of the implementation include the ability to update stored reference patterns at will so as to incorporate the leatures of new utterances, and a graphic display that effectively illustrates which portions of a test utterance are within the programmed limits of acceptability.
\end{abstract}

PP2. Text-Independent Speaker Recognition. B. S. Atal, Bell Telephone Laboratories, Incorporated, Murray Hill, New Jersey 07974.-In automatic speaker recognition methods, the speaker to be recognized is usually required to speak the same utterance which was used to obtain the reference pattern for that speaker. However, such a restriction is not generally necessary for speaker recognition by humans. Is reliable automatic speaker recognition similarly possible? A speaker identification experiment was performed in a population of 10 fernale speakers. The acoustical information was represented by sets of 12 predictor coefficients obtained by minimizing the mean-squared prediction error over speech segments 50 msec in duration. Each set of these predictor coefficients was represented by a 12-dimensional vector. New sets of coordinates which minimized the intraspeaker variance were determined by linear transformation of the original vector space. The segment of the utterance used to identify an individual was different from the segments used to form the reference pattern for that individual. For each segment, the unknown vector was correlated with the reference vectors and the correlations were averaged over a number of segments-the speaker with the largest correlation was identified as the unknown speaker. The over-all identification accuracy was $93 \%$ for 40 speech segments. These results suggest that successful automatic speaker identification is possible independently of the spoken text.
PP3. On Evaluating Formant Synthesizer Control Signals. II. W. WATheN-DunN, S. B. MichlaELS, L. V. KRIGER, AND H. I. Soron, Air Force Cambridge Research Laboratories, Bedford, Massachusetts 01730.-An attempt to evaluate the effectiveness of three sets of formant synthesizer control signals, derived by different means, has utilized VCV utterances in which the C's were one of the six stop consonants and the V's were one of the six vowels, $/ i, e, a, o, u, \Lambda /$. The stops are the most quickly articulated phonemes and the first five of the vowels are tense and are thus articulated at positions most removed from the central articulatory configuration, which is represented by the last vowel, $/ \Lambda /$. Use of these utterances is predicated on the idea that the stops and the tense vowels make the greatest demands on synthesizer performance and are hence most suited for evaluation. Two tests have been run: (1) an identification test for each of the consonants and (2) comparisons of the same consonant produced by different means. Results for the labial stops, $/ \mathrm{b}, \mathrm{p} /$, were presented at the 82 nd Meeting of the Society in Denver, and this paper will present the results for the remaining stops, $/ \mathrm{d}, \mathrm{t}, \mathrm{g}, \mathrm{k} /$.

PP4. Orientation to Speech Sound. T. F. MyERS, Department of Psychalogy, University of Edinburgh, Great Brilain, R. Zhukova and L. A. Chistovich, Pavlov Pyhsiological Institute, Leningrad, U.S.S.R.-A -eries of experiments are described in support of a model of speech recognition. The vowel /a/ from a complex signal generator was presented binaurally, each ear channel being independently controlled for signal intensity and duration of a medial silent interval (0-90 msec), such that a fused percent $/ \mathrm{a} /, / \mathrm{ara} /, / \mathrm{ala} /$, or $/ a-a /$ resulted in the center, or to one or other side of auditory space. Analysis of categorical response probability and lateralization of a percept as functions of duration of the interval and interaural intensity difference enabled the functional levels and operations represented by the model to be substantiated.

PP5. Spoken Digit Recognition Using Partial Autocorrelation Coefficients (PAC). I'. Nakano, A. ICHIKawa, AND K. Nakata, Central Research Laboralory of Hitachi Lid., Kokubunji, Tokyo, Japan.-Spoken digit recognition using autocorrelation coefficients (PAC or $k$ parameters) is investigated with a digital computer. Speech samples are 10 spoken 
Japanese digits uttered by a single male speaker. Each digit is uttered 10 times. Each sample is analyzed at every $10 \mathrm{msec}$ to give time series of PAC. Principle of recognition is a simple pattern matching with nonlinear time adjustment by DP procedure. When the dimension of PAC is greater than 4 , recognition score is fairly good, but does not exceed the value of $98 \%$. The cause of error seems to be the accuracy deterioration of computation of PAC. The computation of PAC is strongly correlated to a signal amplitude. Weighting PAC by amplitude information, the recognition score is improved and reaches at $100 \%$, with the dimension of PAC greater than 4 . When PAC is computed by fixed-point arithmetic with short accumulator length, the score become worse with the increase of the dimension. Further improvement of recognition score and time is expected by the variable use of PAC dimension controlled by the computation accuracy.

PP6. Analysis of Nasal Consonants. G. FANT and Ylva Ertkson, Department of Speech Communication, Royal Institute of Technology, S-100 44 Stockholm, Sweden.--Spectral analysis has been made of 12 Swedish speakers' nasal consonants $/ \mathrm{m} /$ and $/ \mathrm{n} /$ followed by four different vowels. Typical patterns of nasal murmur and assimilated nasality in the vowel portion are discussed with respect to established pole-zero models and results from earlier studies including sweep frequency measurements of the vocal and nasal tract. The perceptual significance of various pattern aspects are discussed.

PP7. The Effect of Nasal Oral-Cavity Uncoupling on the Sound Levels of Selected Stimuli. R. L. Zimmerman AND M. Hoshiko, Department of Speech Pathology and Audiology, Southern Illinois University, Carbondale, Illinois 62908.Sound level measurements of five selected narrow-band noises $30-\mathrm{Hz}$ wide (center frequencies at $250,730,1090$, and $2440 \mathrm{~Hz}$ and $4 \mathrm{kHz}$ ) and two pure tones $(250$ and $500 \mathrm{~Hz}$ ) were made at the external aperture of the left nostril of each of 12 trained male subjects. A miniature transducer, positioned in the oral cavity of the subjects was used for stimulus presentation. Measurements were made during each of two subject generated physiological states. The first measurement was made when the subject's oral and nasal cavities were in open communication. A second measurement was made with the two cavities separated through velopharyngeal sphincter action. The recorded sound levels for the two conditions for all stimuli were statistically compared. The narrow-band noise with the center frequency at $250 \mathrm{~Hz}$ and the $250-\mathrm{Hz}$ pure tone were found to be statistically different from the other stimuli. In all significant comparisons, the difference values were shown to be indicative of less attenuation of the $250-\mathrm{Hz}$ narrow-band stimulus and of the $250-\mathrm{Hz}$ pure-tone stimulus.

PP8. Dimensional Analysis of a Full Vowel Set. R. D. WRIGHT, National Security Agency, Ft. Meade, Maryland 20755.Previous investigators (Li el al.; Pols et al.; Singh ; Watanabe; Wright and Boehm) have reported on the dimensional analysis of sets of Dutch and English vowels. The present investigation used a vowel set larger than that available in any of the single-language sets previously analyzed. The vowel set used contained all combinations of high-low, back-front, and rounded-unrounded distinctions. Representative spectra were measured for twenty-two vowels and analyzed for principal components. The resulting dimensions of maximum variance are compared to the previously reported physical and perceptual dimensions for vowel sounds.

PP9. Analysis of Tongue Parameters for Vowels. Lloy Rice, Phonetics Laboratory, UCLA, Los Angeles, California 90024. - A computer system is described that will accept a spoken vowel as input and then adjust the area function of a vocal-tract synthesizer to duplicate the input vowel quality as nearly as possible. The synthesizer output is matched against the input vowel spectrum using the Markel inverse filtering system. The program allows the definition of alternative parameter models for controlling the vocal-tract area function and represents a preliminary attempt to simulate the major vocal articulators. Each of three to six parameters is defined in terms of its contribution to the movement of each of the 18 sections used in the line analog synthesizer. Thus, the action of parameter $\mathrm{Pi}$ is defined by the 18 -element vector of weights $W_{i 1}, W_{i 2}, \cdots, W_{i 19}$. Any vocal tract shape within the range of the model is specified by assigning the appropriate value $\mathrm{Pi}$ to each of the parameters. The deflection of the $j$ th section away from the neutral tract position is then given by the sum of products $P_{1} W_{1 j}+P_{2} W_{2 j}+P_{2} W_{\mathrm{s} j}+\cdots$. Various parameter models will be explored to determine their usefulness in providing unique and reasonable tongue shape descriptions.

PP10. The Syllable Nucleus as a Unit of Timing. ILse LeHIste, Department of Linguistics, The Ohio Stale Universily, Columbus, Ohio 43210. - The paper explores the question whether resonants preceding and following a vowel can be considered as part of the syllable nucleus. A set of monosyllabic words was studied, beginning and ending with voiced and voiceless obstruent consonants, and containing syllable nuclei of the following types: $\mathrm{V}$ (long and short), $\mathrm{V}+\mathrm{R}, \mathrm{R}+\mathrm{V}$, and $R+V+R$. Three speakers produced each of the 156 words five times. An analysis of the durations of the segments yields (a) that syllable nuclei consisting of $V+R$ function as a unit with respect to the timing patterns conditioned by the voicing of the following consonant, and (b) that resonants combine with long vowels in such a way that the durational differences between syllable nuclei of the types $V, V+R$, and $R+V$ are below the perceptual level (i.e., below established difference limens for duration). The evidence is considered as supportive of the assumption that timing in speech is organized with reference to major changes in manner of articulation.

PP11. Determination of Articulatory Parameters from the Speech Signal. B. S. Atal and J. E. Hartman, Bell Telephone Laboratories, Incorporated, Murray Hill, New Jersey 07974. - A speech analysis method is presented for obtaining a description of the vocal-tract shape in terms of articulatory parameters. A slightly modifed version of the Stevens and House three-parameter articulatory model is used to specify the area function of the vocal tract. The area function is described by five parameters, namely, the area at the place of maximum constriction, the area of the lip opening, the length of the lip section, and the length of the vocal tract. Two additional parameters are used to characterize the source. Considering the vocal tract as a lossy acoustic tube, the parameters of a 12th order linear predictor for the synthetic specch signal at the output of the tube are obtained. The parameters so obtained are used to predict the current value of a speech sample by forming a linear combination of the preceding 12 samples of natural speech. The mean-squared difference between these predicted values and the actual sample values in natural speech is minimized by iteratively adjusting the seven vocal-tract and source parameters. The parameter values that minimize the error are finally used to describe the vocal-tract shape.

PP12. Estimation of the Vocal-Tract Shape by Optimum Inverse Filter Processing of Speech. H. WakitA, Speech Communications Research Laboratory (SCRL), Santa Barbara, 
California 93101.-It is shown that the filtering process of the optimum inverse filter [J. D. Markel, SCRL Monogr. No. 7, 111 (Oct. 1971)] is identical to the filtering process of the nonuniform acoustic tube model [B. S. Atal and S. L. Hanauer, J. Acoust. Soc. Amer. 50, 637 (1971)] in which the vocal tract is divided into a finite number of sections with equal lengths. The common factor between the two processes is shown to be a set of reflection coefficients. These reflection coefficients are obtained as intermediate results of a recursive solution to the inverse filter equations. Both area functions and filter coefficients can be easily determined from this set of reflection coefficients. The procedure can be realized by recursive digital filters, thus making possible an efficient implementation as a computer program to obtain the time variation of the vocaltract shape together with the spectral envelope of the speech sample. This procedure simplifies finding the relationships between the vocal-tract shape and its acoustic features, e.g., from the vocal-tract shape to the filter coefficients, from which formant frequencies and bandwidths can be determined, and vice versa. Applications and limitations of this procedure to connected speech are discussed. [The work was supported by the Office of Naval Research.]
PP13. More Visible Speech. Janet M. BAkER and James K. BAKER, Rockefeller University, New York, New York 10021, AND JEROME Y. LETTVIN, Massachusetts Institule of Technology, Cambridge, Massachusetts 02 139.-This paper presents a method for visual display of acoustical waveforms with which different phonemes are more readily distinguished than with spectrograms. The visual display is based on the interval between successive up crossings of the zero axis in the waveform. Although zero crossings and up crossings have been used by several investigators since Licklinder's studies demonstrating that zero-crossing information is sufficient for intelligibility of speech, most investigators have ignored an essential property of up-crossing analysis. Analysis of up crossings is a time domain technique and as such, allows a perfect resolution of events in time, a resolution which is lost if the up-crossing data is averaged over intervals of time. Such precise time resolution is critical for the recognition of certain distinguishing features in various consonants. These features permit a visual display in which the phonemes can easily be distinguished, even in connected speech. Furthermore, the important features for discriminating among the consonants are highly speaker invariant.

FRIDAY, 21 APRIL 1972

EMBASSY RoOM, $2: 00$ P.M.

\title{
Session QQ. Psychological Acoustics IV : Hearing Aids and Audiometry
}

\author{
H. N. WRIGHT, Chairman
}

State University of New York, Upstate Medical Center, Syracuse, New York 13210

\section{Contributed Papers (12 minutes)}

\section{2:00}

QQ1. Insert Earphone Pressure Response in Real Ears and Couplers. R. M. SACHS AND M. D. BuRkhaRd, Industrial Research Products, Inc., 321 Bond Street, Elk Grove Village, Illinois 60007.-Pressures measured in five real ears and the standard $2-\mathrm{cm}^{3}$ and Zwislocki couplers have shown that (1) Pressure in Zwislocki coupler is essentially identical to pressure in real ears (with no ear mold leaks) below $500 \mathrm{~Hz}$ implying an equivalent real ear volume of about $1.2 \mathrm{~cm}^{3}$. Pressure in $2-\mathrm{cm}^{\mathrm{a}}$ coupler is about $4 \mathrm{~dB}$ lower. (2) Between 500 $\mathrm{Hz}$ and $5 \mathrm{kHz}$, the real ear minus $2-\mathrm{cm}^{3}$ coupler difference increases with frequency about $2.5 \mathrm{~dB} /$ oct to about $+12 \mathrm{~dB}$ at $5 \mathrm{kHz}$. The real ear minus $Z$ wislocki coupler difference, however, is no more than $\pm 2 \mathrm{~dB}$ up to $5 \mathrm{kHz}$. (3) Above $5 \mathrm{kHz}$, there is evidence that pressure in real ears decreases with frequency relative to both couplers. Real ear pressure was measured using a probe-tube microphone (corrected to read ear drum pressure) ; five types of insert earphones (hearing aid receivers) were used. When dealing with insert earphones whose source impedance is very high relative to the input impedance of the coupler (this is usually the case), it is sufficient to measure the insert earphone pressure on the $2-\mathrm{cm}^{3}$ coupler. Real ear drum pressure can then be determined by applying a correction curve (either graphically or by electronic filtering) to be presented. The Zwislocki coupler would then serve as a laboratory standard for comparison.

\section{2:15}

QQ2. Semantic Labeling of Hearing-Aid Acoustics. S. G. Revorle, Auditory Research Laboratory, Veterans Administration Hospital, Washington, D. C. 20422.-Hearing aids were measured electroacoustically prior to their issuance to 50 hearing-impaired subjects. For each aid, measurements were conducted with the gain of the instrument at the level set by the subject. After one month of use the subjects completed questionnaires concerning the acoustic quality of these hearing aids. The questionnaire included a checklist of 94 descriptive labels previously obtained from the unrestricted linguistic output of hearing-aid users regarding their hearing aids acoustic qualities. Among the labels on the checklist, the subjects marked those which were appropriate for describing "good" quality or "poor" quality, and the quality of their own aids. Subjects showed greater agreement in their choices of labels appropriate for describing poor quality than for describing good quality. The most frequently chosen labels will be discussed, along with presentation of the relationship between these semantic labels and the hearing aids' electroacoustic characteristics.

$$
2: 30
$$

QQ3. On Some Parameters of Hearing Aids with Compression. IGOR V. NÁBĚLEK, Biocommunications Laboratory, Universily of Maryland, College Park, Maryland 20901.Introduction of compression into hearing aids increases the number of variable electroacoustic parameters of hearing aids. The evaluation of the influence of each individual parameter on hearing aid performance is difficult even with ordinary hearing aids. In addition to the variability in the frequency range, regularity of frequency response, harmonic distortion, etc., hearing aids with compression differ in the range of compression, degree of compression, threshold of compression, etc. One of the significant characteristics is the time constant 
of compression. Its importance in hearing aids performance is still to be clarified. In the paper the necessity of good description and specification of this parameter for comparability of measurements in different laboratories will be discussed. The values of the release and attack durations, and of overshoots, as determined by measurement, are dependent on the choice of several parameters. The influence of the hearing aid volume control setting, of the operating point on the inputoutput curve, of the range of the input SPLs, and of frequency will be discussed and shown in several examples.

\section{$2: 45$}

QQ4. A Factor Analysis of Competing Message Testing in Hearing Aid Evaluations. Daniel L. Bode, Elizareth F. Dodds, and Raymond Carhart, Auditory Research Laboratories, Northwestern University, Evanston, Illinois 60201.Retrospective data compiled from routine clinical hearing aid evaluations were examined to identify the primary factors influencing test performance of hearing-impaired listeners. Results were analyzed from representative and similarly conducted evaluations with 68 patients. Unaided test measurements were obtained bilaterally, and included pure-tone thresholds, slope of the audiometric contour, speech reception thresholds (SRT), and speech discrimination scores. Aided tests included speech reception threshold, speech discrimination in quiet, and speech discrimination against a competing message. W' hen intercorrelations among 27 test variables were subjected to factor analysis, five factors emerged: speech discrimination, nontest ear thresholds, unaided sensitivitytest ear, interear high frequency thresholds, aad aided SRT. The 68 sets of data were subdivided into two groups on the basis of severity of hearing impairment, and new factor analysis were run. Here the same array of factors emerged with the addition of (1) a nontest ear high-frequency factor for the milder loss subgroup and (2) two factors designated as SRT-poorest aided performance, and discriminationpoorest aided performance for the greater loss subgroup.

\section{3:00}

QQ5. Discrimination Loss and Masking by Low-Frequency Vowel Formants. E. Martin, M. J. Osberger, AND J. M. Picketr, Gallaudet College, Washington, D. C. 20002.-Most persons with sensorineural hearing loss have some impairment of speech discrimination ability. In most cases, discrimination of consonants is poorer than discrimination of vowels. This study investigates the ability of hearing-impaired Ss to discriminate one type of consonant cue-a vowel formant transition. Our purpose was to study the effect of the presence of $F 1$ on discrimination of $F 2$ transitions. The test stimuli were synthetic vowels whose spectra approximated those of [a] and [i]; they were 250 msec in duration. When an $F 2$ transition was presented, it occurred over the initial $100 \mathrm{msec}$, after which $F 2$ did not change. The Ss were 23 persons with moderate to severe sensorineural hearing loss and four normalhearing persons. Results: Some hearing-imparied Ss showed essentially normal discrimination with $F 1$ present. However, in most of the Ss, $F 1$ produced large amounts of masking in both [a] and [i]. The amount of masking was greater in [i] than in [a]. In this respect, the effect is not the same as the "classical" upward spread of masking. When the normal Ss were tested at sound levels approximating those used to test the hearing-impaired Ss, they showed large amounts of masking. The highi sound levels at which hearing-imparied persons must listen appear to be a factor in their discrimination loss. [Work supported partly by the U. S. Public Health Service.]

\section{3:15}

QQ6. Speech Feature Perception by Listeners with Severe Sensorineural Hearing Loss. J. M. Picketr and Ellen S. Martin, Gallaudel Callege, Washington, D. C. 20002.-How well do persons who show discrimination loss for speech perceive the different phonetic features, i.e., consonant voicing, place and manner of articulation, and vowel features? Would the pattern of feature perception be radically different between groups of listeners with different amounts and frequency contours of hearing loss? We present preliminary answers to these questions based on group confusion matrices of the responses to a speech perception test given to $99 \mathrm{im}$ paired listeners. Results show that the feature of place of consonant articulation is heard more poorly than the features of voicing and low-frequency continuance, over a rather wide range of listener groups. Groups formed according to frequency contour of hearing loss showed some significant, but relatively small, differences in feature reception.

\section{$3: 30$}

QQ7. A New System for Performing Sensorineural Acuity Level (SAL) Testing. James T. Yates, Speech and Hearing Clinic, Texas Tech Universily, Lubbock, Texas, 79409.-A system for SAL testing has been developed which alleviates the problems previously experienced with SAL-Rainville procedures. The occlusion effect, the primary problem area in SAL testing, was circumvented through the design of a nonoccluding earphone which incorporated an especially constructed miniature transducer. The use of a narrow-band masking effectively extended the useful range of the SAL. procedure. The system is now in limited clinical and research application.

\section{$3: 45$}

QQ8 Pure-Tone Masking in Normal and Cochlear Impaired Ears. Alan M. Richards, Communication Sciences Laboratory, Department of Speech, Herbert H. Lehman College of the City University of New York, Bronx, New York 01468.--Pure-tone masking patterns were obtained using equivalent sensation levels (SLs) of a $500-\mathrm{Hz}_{z}$ masking tone in both normal hearing and cochlear impaired subjects. When the level of the masker was set at a relatively low intensity ( $35 \mathrm{~dB}$ SL), the two groups yielded equivalent threshold shifts at all of the masked frequencies (125-8000 $\mathrm{Hz}$ in octave increments). However, when the masker level was increased to $55 \mathrm{~dB} \mathrm{SL}$, it was found that those cochlear impaired subjects who exhibited partciular audiometric criteria below $2000 \mathrm{~Hz}$ (e.g., $20 \mathrm{~dB}$ HL threshold at $500 \mathrm{~Hz}$ ) generally showed greater threshold shifts at 1000 and $2000 \mathrm{~Hz}$, while those impaired subjects not meeting these criteria yielded masking patterns that differed in no essential manner from the normals. These results were evaluated relative to those studies that have used narrow-band noise to investigate the spread of masking phenomena in normal and pathological ears.

\section{4:00}

QQ9. Pure-Tone Octave Masking in Listeners with Sensorineural Hearing Loss. R. C. Brlger and D. A. Nelson, Bioacoustics Laboratory, Eye and Ear Hospital, University of Piltsburgh, Pittsburgh, Pennsylvania 15213.-Phase-locked pure-tone masking was investigated in 16 listeners with sensorineural hearing loss to examine the hypothesis that the sensorineural ear introduces abnormal distortion. Thresholds for a pure tone at $f_{2}$, masked by a pure tone at $f_{1}$ (where $f_{2}=2 f_{1}$ ), were obtained as a function of the level of the $f_{1}$ masker for three different $f_{1}$ frequencies $(250,500$, and 1000 $\mathrm{Hz}$ ) and two different phase relations between $f_{1}$ and $f_{2}$ 
$\left(90^{\circ}\right.$ and $\left.270^{\circ}\right)$. Estimates of the level of the aural harmonic were obtained from those masking functions. Analysis of the data in terms of the absolute level of $f_{2}$ at masked threshold versus the absolute level of $f_{1}$ results in a conclusion that these pathological ears do not perform differently from normal ears, except along the dimension of hearing loss. That is, their hearing loss does not add significant distortion to the acoustic signal. Even though the present analysis suggests that evaluation of the data for pure-tone octave masking in terms of SL leads to the specious conclusion that the sensorineural ear introduces abnormal distortion, an attempt was made to relate hearing loss to the aural harmonic in less simplistic terms [Research supported by grants from the NINDS of USPHS.]

$$
4: 15
$$

QQ10. Contralateral Masking Effects on Auditory Thresholds. GAIl STIFFel AND I. M. Young, Thomas Jefferson University, Philadelphia, Pennsylvania 19107.-Thresholds and amplitude measurements of fixed-frequency Bekesy tracings were made in the presence of contralateral white-noise masking using bilateral normal-hearing subjects, and subjects with unilateral sensorineural hearing loss. In bilateral normal ears, the thresholds for both interrupted and continuous tones increased linearly as the cross masking effect increased. Amplitude remained unchanged. In some cases of unilateral sensorineural hearing loss, when the pathological ear was masked the noraml ear demonstrated threshold shif $t$ and reduction in amplitude for the continuous tone when the intensity level of the noise was too low to be attributed to cross masking. On the other hand, when the normal ear was masked, the first group showed the threshold shift and reduction in amplitude for continuous tones when the masking noise was less than the interaural attenuation. The second group demonstrated similar findings when noise levels were greater than the interaural attenuation In both groups, these changes were either abrupt and plateaued or gradually increased with increased masking. There were insignificant changes for interrupted tones. The question still remains whether the shift in the threshold and amplitude of the continuous tones is due to (1) central masking, (2) cross masking, (3) pathological condition.

$$
4: 30
$$

QQ11. Effects of Testing Methods on Békésy Thresholds. I. M. Young, Department of Otolaryngology, Thomas Jefferson University, Philadephia, Pennsylvania 19107.-Thresholds and amplitude measurements of fixed-frequency Békésy tracings were made for continuous tones using the method of adjustment on normal hearing subjects. Attenuation rates were 1 , 2,4 , and $8 \mathrm{~dB} / \mathrm{sec}$ with $0.2 \mathrm{~dB}$ steps. Results indicated that while the thresholds measured at the midpoints of amplitudes were practically constant with differ-attenuation rates, the thresholds at the tops and the bottoms of amplitudes showed a distinct difference for chauging attenuation rates. As attenuation rates increased, the thresholds at the tops of amplituded improved and the thresholds at the bottoms of amplitudes became worse. Amplitudes increased in size as attenuation rate increased but there was no constant correlation between amplitude and attenuation rate. Conventionally, the midpoint of the Békséy ampliturle has been considered the best estimate of the threshold. This is true only when the Békésy audiogram was obtained by using the method of adjustment.

$$
4: 45
$$

QQ12. Auditory Thresholds using a Single Sweep Frequency Modulation. I. M. Young and C. Wenner, Department of Otolaryngology, Thomas Jefferson University, Philadelphia, Pennsylvania 19107.-Békésy thresholds were obtained for frequency-modulated tones in normal-hearing subjects. Measurements were made at a fundamental frequency of $1000 \mathrm{~Hz}$ by using combination of four frequency deviations, four "on times," and three "off times." In each case, the on time consisted of a single sweep of frequencies. Attenuation rate of Békésy-type recording attenuator was $1 \mathrm{~dB} / \mathrm{sec}$. Results indicated that for a given frequency deviation, the thresholds improved with longer on time (slow sweep of frequencies) and longer off times. For a given on time, as frequency deviation increased the threshold improved. Combination of long on time, long off time, and wide frequency deviation resulted in the best threshold. 